\title{
Trends in relevant excess mortality in Peru and nine areas during the first wave of COVID-19 pandemic between January and May 2020: A descriptive study
}

\section{Juan Diego}

Universidad Peruana Cayetano Heredia https://orcid.org/0000-0002-1127-0028

Josè Enrique ( $\nabla$ j.enrique.v1ton.rub1o@gmail.com )

Universidad Peruana Cayetano Heredia https://orcid.org/0000-0001-9245-7726

\section{Research Article}

Keywords: Mortality, COVID-19, Public Health Surveillance, Peru

Posted Date: January 22nd, 2021

DOI: https://doi.org/10.21203/rs.3.rs-142440/v1

License: (c) (i) This work is licensed under a Creative Commons Attribution 4.0 International License.

Read Full License 
1 Trends in relevant excess mortality in Peru and nine areas during the first wave of

2 COVID-19 pandemic between January and May 2020: A descriptive study

3 Authors:

4 Mendoza-Saldaña Juan-Diego ${ }^{1}$ https://orcid.org/0000-0002-1127-0028

5 Viton-Rubio J. Enrique ${ }^{1}$ https://orcid.org/0000-0001-9245-7726

$6 \quad{ }^{1}$ School of Medicine Alberto Hurtado, Peruvian University Cayetano Heredia, Lima, Perú 7

9

10 Corresponding author: Vitón-Rubio José-Enrique

11 Institutional address: Av. Honorio Delgado 430, 15102, Lima, Perú

12 Phone: +51939286236

13 E-mail: j.enrique.v1ton.rub1o@gmail.com

14

15

16 Abbreviated title: Trends in relevant excess mortality in Peru and nine capitals during COVID-

1719 pandemic.

18 
ABSTRACT

Introduction: COVID-19 has arrived the main capitals of Peru and caused direct and indirect deaths. Though mortality data is reported through SINADEF, relevant excess mortality (REM) remains unknown.

Objective: To estimate the monthly relevant excess mortality for all causes and non-related with violent and COVID-19 causes between January and May 2020 in Peru and nine areas with the highest absolute numbers of deaths by COVID-19.

Material and Methods: To estimate relevant excess mortality, we calculated the monthly upper and lower limits of historical mortality (95\% confidence interval) and the average for the last 3 years (2017-2019). REM was considered when observed values exceed the estimated upper limit plus corresponding 2 standard deviations. We also determined REM excluding nonrelated with violent and COVID-19 causes.

Findings: Relevant excess mortality of 35 deaths per 100000 population from all causes during April and May 2020 were found in Peru with no difference when we excluded violent deaths. In addition, Callao had the highest REM followed by Lima, Ucayali, Loreto, Piura, Ica, Ancash, and La Libertad. In country and capitals analysis, male and individuals over 60 years were the most affected groups.

Discussion: Relevant excess mortality determination is a needed, easy and low-cost approach to monitor the progress of COVID pandemic and to evaluate the effectiveness of policy measures. This surveillance method should expand to the 25 locations of Peru.

Conclusion: COVID-19 accounted for $45 \%$ of the relevant excess all-cause deaths, leaving $54.29 \%$ unattributed to COVID-19. Hence, it is necessary to rethink the policy measures in order to avoid direct and indirect deaths attributed to the pandemic. 


\section{INTRODUCTION}

The severe acute respiratory syndrome coronavirus 2 (SARS-CoV-2) first emerged in

December 2019 in Wuhan, China and rapidly spreads across most countries of the world(1). The Coronavirus Disease 2019 (COVID-19) was confirmed to have reached Latin America in February 2020 and Peru reported its first case on March 6, 2020(1,2). The World Health Organization (WHO) even recognized that COVID-19 was a pandemic on March 11(1). On March 15, the Peruvian government just declared a national state of emergency and meanwhile there had already reported 71 cases in the main cities of Peru(2).

The first death was reported on March 19 and at the end of the month there were already 66 deceases: $75.75 \%$ men and $66 \%$ over 60 years old(3). At the end of May, Peru was the tenth most affected country with 178024 infected people and 4638 deaths(2,3). Although there was a considerable increase of molecular and serological testing for COVID-19, 11518 molecular tests were only performed between March 4 and March 30(4). With the limited availability of tests and imperfect sensitivity of the serological tests(5), there have indisputably been a number of deaths caused by COVID-19 that are not counted in official data. Indirect deaths are also common in a pandemic situation due to an overloaded health system or lack of healthcare access (6). Both types of death are described in specific risk groups.

A meta-analysis of thirteen studies found that male, older than 65 years, current smoking were risk factors for severe disease progression in patients with COVID-19(7). It can also aggravate and increase mortality burden especially affecting people with prior health conditions including diabetes, hypertension, cardiovascular disease, and respiratory disease (7). In particular, Peru has epidemiological features in relation to severe risk factors. 
77 According to the last Peruvian national census in 2017, the population accounted for 29381

884 inhabitants with almost one third of the nation's population living in its capital, Lima(8).

Moreover, $49.18 \%$ of individuals were male and only $11.9 \%$ were over 60 years old(8).

Comorbidities are common and it was reported that $3.6 \%, 9.5 \%$ and $22.7 \%$ of the population

over 15 years old were diagnosed with diabetes, hypertension and obesity respectively in 2018(9). Additionally, $11.2 \%$ of people smoke cigarettes currently in 2018(9). Indeed, Peruvian data reported older than 60 years and patients with hypertension and obesity had a greater risk of death $(10,11)$.

On the other hand, secondary consequences of social distancing may increase suicides, homicides and decrease motor vehicle accidents. For example, canceled public events and closed businesses have been associated with higher suicide rates compared with times of prosperity (12). Also, lockdown could interrupt the access to community and religious support or mental health treatment and raise this kind of violent deaths(13). In order to concentrate on biological causes of death, we excluded violent deaths.

92

Weekly excess deaths analysis could provide the most objective and comparable way of assessing the total mortality effects of a pandemic (16). Unfortunately, most countries do not publish such statistics. National Death Registry Information System (SINADEF) provides a monthly number of deaths.

Therefore, to track the impact of COVID-19 in Peru, we estimated the monthly excess mortality for all causes and non-related with violent and COVID-19 causes between January and May 2020 Peru and nine areas with the highest absolute numbers of deaths by COVID-19. 


\section{METHODS}

103 This is a descriptive study based on official public data in which we analyzed the excess of

104 deaths between January 1 and May 31 of 2020 by all causes and excluding the violent and

105 COVID-19 deaths

\section{Study locations}

107 Peru's divisions are 25 areas: 24 departments and one constitutional province(Callao) of which 108 we selected the areas with the highest absolute numbers of deaths by COVID-19 (>100 deaths) 109 until May 31th, 2020 according a study that found the excess was greater in the regions most 110 affected by COVID-19 in Italy (14). These were Lima, Lambayeque, Piura, Loreto, Callao, 111 Ancash, Ica, La Libertad and Ucayali.

\section{Data sources}

114 We accessed the mortality database updated to Jun 6th, 2020 from SINADEF (15). The data

115 included the monthly number of deaths by sex and age class. We also used data from violent 116 deaths, namely those associated with traffic accidents, homicides, suicides, work-related deaths

117 and other accidents. Data are available for the period January 1st to May 31th, 2020, and for 118 the corresponding periods in 2017-2019.

120 Data from confirmed deaths by COVID-19 updated to Jun 6th, 2020 were collected from the 121 National Open Data Platform (NODP) (3). The published data corresponds date of birth, date 122 of death, sex, department, province and district. We did not use province and district data. 123 Although, SINADEF and NODP have missing data on subtotal deaths (unknown age or gender). We did our best to mitigate the impact of these data quality issues. 
126 Current population data was not available. Demographic data were collected from the 2017

127 Peruvian National Census (8). We only obtained general, sex and age groups population data

128 to calculate the mortality rates.

129

\section{Data analysis}

131 To estimate the excess mortality (EM), we calculated the monthly upper and lower limits of

132 historical mortality (95\% confidence interval) and the average for the last 3 years (2017-2019)

133 and the current year between January 1st and May 31th because the information was not 134 available for earlier years.

136 The Relevant Excess Mortality (REM) calculation method has been considered when the 137 observed values exceed the estimated average value plus corresponding 2 standard deviations $138(17,18)$. Our approach is more careful to not overestimate the excess of mortality. We consider

139 REM when the observed values exceed the estimated upper limit value plus corresponding 2 140 SDs but we also show the REM related to the estimated average value. We calculated REMs 141 using death absolute values and mortality rates to total deaths (TD), total deaths excluding

142 violent deaths (TD-VD) and COVID-19 deaths (TD-VD-CD). The same process was done by 143 sex and age class $(0-11 y, 12-17 y, 18-29 y, 30-59 y$ and $\geq 60 y)$.

144

145 Data were downloaded as tables and analyzed using Microsoft Excel 19. We developed a data 146 extraction sheet. One author extracted data, the second author checked the extracted data and 147 both analyzed the information. Figures were designed using STATA 16 licensed by Cayetano 148 Heredia Peruvian University. 
150 No information at the individual level or on the cause of death was used, therefore clearance

151 from the Ethical Review Board was not necessary.

152

\section{RESULTS}

\section{Peru}

155 Our results were compatible with no REM between January-March 2020 and REM of 6 and 29 156 deaths per 100,000 population in April and May 2020 severally (Table 1 and fig. 1). During 157 January and February, Peru did not informed COVID-19 deaths. This study did not find a 158 difference in April and May REM when we evaluated TD-VD. In the evaluation of TD-VD$159 \mathrm{CD}$, our findings only indicated REM of 19 deaths per 100,000 population in May. Hence, 160 COVID-19 deaths explained fully April REM and partially May REM.

162 We noted REM for men of 11 and 44 deaths per 100,000 population for April and May 2020 respectively (Table 2 and fig. 2). Additionally, this study did not find difference when we evaluated TD-VD and just 1 and 31 deaths per 100,000 population in April and May respectively for TD-VD-CD. On the other hand, this study manifested REM for women of 1 death per 100,000 population and 14 deaths per 100,000 population in April and May respectively (Table 2 and fig. 3). We observed the same REM when we evaluated TD-VD and just 9 deaths per 100,000 population in May for TD-VD-CD.

170 We did not find REM between January and March 2020 by age group (table 3 and Fig. 4-6).

171 April REM was 5 deaths per 100,000 population for the 30-59y group and 21 deaths per 100,000

172 population for $\geq 60 y$ group (table 3 and Fig. 7). May REM was 30 deaths per 100,000

173 population for the $30-59 y$ group and 188 deaths per 100,000 population for $\geq 60$ yroup (table

1743 and Fig. 8). Note that when we assessed TD-VD, it did not represent a significant difference 
175 in REM. Otherwise, we did not find REM if we evaluated TD-VD-CD in April. Instead, we 176 found REM of 22 and 130 deaths per 100,000 for the $30-59 y$ group and $\geq 60 y$ group respectively 177 in May.

\section{Lima}

180 Our results are compatible with REM of 4, 25 and 59 deaths per 100,000 population in March, 181 April and May 2020 severally (Table 1 and Fig. 9). This study did not find difference in REM 182 when we evaluated TD-VD. In the evaluation of TD-VD-CD, our findings indicated REM of 3, 17 and 48 deaths per 100,000 population in March, April and May respectively. We found REM for men of 5, 35 and 86 deaths per 100,000 population in March, April and May 2020 respectively (Table 2 and Fig. 10). Additionally, this study found just a little difference in March and April when we evaluated TD-VD and just 3, 23 and 73 deaths per 100,000 population in March, April and May severely for TD-VD-CD. On the other hand, this study manifested REM for women of 4, 15 and 32 deaths per 100,000 population in March, April and May respectively (Table 2 and Fig. 11). We observed the same REM when we assessed TD-VD and just 3, 11 and 28 deaths per 100,000 population in March, April and May severally for TD-VD-CD.

We find REM in all the months by age group, except in February 2020 (Table 3 and Fig. 13).

194 January REM was 1 death per 100,000 population for the $18-29 y$ group (Table 3 and Fig. 12). 195 In March we observed REM of 31 deaths per 100,000 population for $\geq 60$ yroup (Table 3 and 196 Fig. 14). April REM was 14 and 107 deaths per 100,000 population for the 30-59y group $\geq 60 y$ 197 group respectively (Table 3 and Fig. 15). May REM was 52 deaths per 100,000 population for 198 the 30-59y group and 358 deaths per 100,000 population for $\geq 60 y$ group (Table 3 and Fig. 16). 199 Note that when we assessed TD-VD, it did not represent a significant difference in REM. If we 
evaluated TD-VD-CD, March REM was 27 per 100000 population for $\geq 60 y$ group; April REM was 8 deaths and 66 deaths per 100000 population for the 30-59y and $\geq 60 y$ group severally. May REM was 44 deaths per 100,000 population for the 30-59y group and 298 deaths per 100000 population for $\geq 60 \mathrm{y}$ group when we assessed TD-VD-CD.

\section{Lambayeque}

Our findings showed that all deceases were within the expected parameters from January to May 2020. Though, we could see a constant progression until March around 3.42 and 4.18 deaths per 100,000 population and a growing linear trend since April which passed from 22.8 to 54.04 deaths per 100,000 population in May (Fig. 17). Note that SD for 2017-2019 monthly values $(20-26)$ was higher than other areas $(<12)$ (Table 1$)$.

Similar situation happened with sex, but male had a higher slope than women from March to May 2020 (Fig. 18 and 19). For age groups, we observed a constant tendency from January to March for all age groups (Fig. 20-22). In April and May, the three first groups had a constant tendency; but the 30-59y and $\geq 60 y$ groups had an increasing linear tendency (Fig. 23 and 24). Note that individuals $\geq 60 \mathrm{y}$ was the most affected set.

\section{Piura}

Our results are only compatible with REM of 40 per 100,000 population in May (Table 1 and Fig. 25). This study did not find difference in REM when we evaluated TD-VD. In the evaluation of TD-VD-CD, our findings indicated REM of 21 deaths per 100,000 population in May.

We found REM for men of 3 and 62 deaths per 100,000 population in April and May 2020 respectively (Table 2 and Fig. 26). Additionally, this study found just a little difference in April 
when we evaluated TD-VD and just 37 deaths per 100,000 population in May severely for TDVD-CD. On the other hand, this study manifested REM for women of 18 deaths per 100,000 population in May (Table 2 and Fig. 27). We observed the same REM when we assessed TDVD and just 7 deaths per 100,000 population in May for TD-VD-CD.

We did find REM between January and March 2020 by age group (Table 3 and Fig. 28-30). April REM was only 14 deaths per 100,000 population for the 30-59 years group (Table 3 and Fig. 31). In May, we observed REM of 1, 46 and 268 deaths per 100,000 population for the 1829y, 30-59y and $\geq 60 y$ group respectively (Table 3 and Fig. 32). Note that when we assessed TD-VD, it did not represent a significant difference in REM for April and May. If we evaluated TD-VD-CD, May REM was only 28 deaths per 100000 population for the 30-59y group and 158 deaths per 100,000 population for $\geq 60$ group.

\section{Loreto}

Our results are compatible with REM of 4, 8 and 45 deaths per 100,000 population in February, April and May 2020 severally (Table 1 and Fig. 33). This study found a little difference in February REM and no difference in April and May REM when we evaluated TD-VD. In the evaluation of TD-VD-CD, our findings only indicated REM of 3 and 24 deaths per 100,000 population in February and May respectively.

We found REM for men of 2, 15 and 64 deaths per 100,000 population in February, April and May 2020 respectively (Table 2 and Fig. 34). Additionally, this study found just a little difference when we evaluated TD-VD and just 1 and 34 deaths per 100,000 population in February and May severally for TD-VD-CD. On the other hand, this study manifested REM for women of 5 and 26 deaths per 100,000 population in February and respectively (Table 2 and 
Fig. 35). We observed the same REM when assessed TD-VD and just 15 deaths per 100,000 population in May for TD-VD-CD.

We found REM in all months by age groups, except in March 2020 (Table 3 and Fig. 38). January REM was 4 deaths per 100000 population for the 30-59y group (Table 3 and Fig. 36). In February we observed REM of 3 deaths per 100,000 population for $\geq 60$ y group (Table 3 and Fig. 37). April REM was 13 and 74 deaths per 100,000 population for the $30-59 y$ and $\geq$ 60y group severally (Table 3 and Fig. 39). May REM was 62 and 418 deaths per 100,000 population for the 30-59y and $\geq 60 y$ group respectively (Table 3 and Fig. 40). Note that when we assessed TD-VD, it did not find REM in February; but a little difference in REM in the other months. IF we evaluated TD-VD-CD, it did not find REM in April. However, May REM was 43 and 252 deaths per 100,000 population for the $30-59 y$ and $\geq 60 y$ group severally when we assessed TD-VD-CD.

\section{Callao}

Our results are compatible with REM of 25 and 94 deaths per 100,000 population in April and May 2020 severally (Table 1 and Fig. 38). This study found a little difference when we evaluated TD-VD. In the evaluation of TD-VD-CD, our findings indicated REM of 12 and 73 deaths per 100,000 population in April and May respectively.

We found REM for men of 39 and 127 deaths per 100000 population in April and May 2020 severally (Table 2 and Fig. 39). Additionally, this study found just a little difference in April when we evaluated TD-VD and just 19 and 102 deaths per 100000 population in April and

273 May respectively for TD-VD-CD. On the other hand, this study manifested REM for women 274 of 8 and 60 deaths per 100000 population in April and May 2020 (Table 2 and Fig. 40). We 
observed the same REM when we assessed TD-VD and just 2 and 49 deaths per 100000 population in April and May severally for TD-VD-CD.

277

We did find REM between January and March 2020 by age group (Fig. 41-43). April REM was 20 and 119 deaths per 100000 population for the 30-59y and $\geq 60 y$ group (Table 3 and Fig. 44).

In May 2020 we observed REM of 68 and 603 deaths per 100000 population for 30-59y and $\geq$ 60y group respectively (Table 3 and Fig. 45). Note that when we assessed TD-VD, it did not represent a significant difference in REM. If we evaluated TD-VD-CD, April REM was 9 and 54 deaths per 100000 population for the 30-59y and $\geq 60 y$ group; and May REM was 51 and 491 deaths per 100000 population for the $30-59 y$ and $\geq 60 y$ group respectively when we assessed TD-VD-CD.

\section{Ancash}

Our results are compatible with REM of 22 per 100,000 population in 2020 May (Table 1 and

Fig. 46). This study found little difference in REM when we evaluated TD-VD. In the evaluation of TD-VD-CD, our findings only indicated REM of 3 deaths per 100,000 population were observed in May.

We found REM for men of 46 deaths per 100000 population for 2020 May (Table 2 and Fig. 47). Additionally, this study found just a little difference in April when we evaluated TD-VD and just of 21 deaths per 100000 population in May for TD-VD-CD. On the other hand, we 296 did not observe REM for women. 
3 and Fig. 53). Note that when we assessed TD-VD, it did not represent a significant difference in REM for April and May. If we evaluated TD-VD-CD, May REM was of 7 and 42 deaths per 100000 population for $30-59 y$ and $\geq 60 y$ group severally.

\section{Ica}

Our results are compatible with REM of 34 per 100,000 population in May 2020 (Table 1 and Fig. 54). This study found a little difference when we evaluated TD-VD. In the evaluation of TD-VD-CD, our findings only indicated REM of 10 deaths per 100,000 population in May.

We found REM for men of 12 and 48 deaths per 100000 population in April and May 2020 severally (Table 2 and Fig. 55). Additionally, this study found just a little difference in April and May when we evaluated TD-VD and just 20 deaths per 100000 population in May for TDVD-CD. On the other hand, this study manifested REM for women of 14 deaths per 100000 population in May 2020(Table 2 and Fig. 56). We observed the same REM when we assessed TD-VD. We did not find REM in May for TD-VD-CD

316 We found REM in all months by age groups, except in March 2020 (Table 3 and Fig. 59). January REM was 3 deaths per 100000 population for 12-17y group (Table 3 and Fig. 57). In February we observed REM of 1 death per 100000 population for the 18-29y group (Table 3 and Fig. 58). April REM was 1 death per 100000 population for 12-17y group (Table 3 and Fig. 60). May REM was 33 and 180 deaths per 100000 population for the $30-59 y$ and $\geq 60 y$ group respectively (Table 3 and Figure 61). Note that when we assessed TD-VD, it did not find REM in February and April; but a little difference in REM for January and May. If we evaluated 
was 10 and 54 deaths per 100,000 population for the $30-59 y$ and $\geq 60$ y group respectively when we assessed TD-VD-CD.

\section{La Libertad}

Our results are compatible with REM of 11 deaths per 100,000 population in May 2020 (Table 1 and Fig. 62). This study found a little difference in REM when we evaluated TD-VD. In the evaluation of TD-VD-CD, our findings only indicated REM of 2 deaths per 100,000 population in May.

We found REM for men of 17 deaths per 100000 population in May 2020 (Table 2 and Fig. 63). Additionally, this study found just a little difference in May REM when we evaluated TDVD. In the evaluation of TD-VD-CD, our findings only indicated REM of 4 deaths per 100000 population in May. On the other hand, this study manifested REM for women of 3 deaths per 100000 population in May (Table 2 and Fig. 64). We observed little difference in REM when we assessed TD-VD and no REM for TD-VD-CD.

We did not find REM between January and April 2020 by age group (Table 3 and Fig. 65-68). May REM was 12 and 68 deaths per 100000 population for 30-59y and $\geq 60$ y group respectively (Table 3 and Fig. 69). Note that when we assessed TD-VD, it did not represent a significant difference in REM for April and May. If we evaluated TD-VD-CD, May REM was 4 and 17 deaths per 100000 population for the 30-59y and $\geq 60 y$ group severally.

\section{Ucayali}

Our results are compatible with REM of 30 and 51 per 100,000 population in 2020 April and May severally (Table 1 and Fig. 73). This study did not difference in REM when we evaluated 
TD-VD. In the evaluation of TD-CD-CD, our findings only indicated REM of 20 and 40 deaths

350 per 100,000 population in April and May respectively.

352 We found REM for men of 80 deaths per 100,000 population in May 2020 (Table 2 and Fig. 353 74). This study did not difference in REM when we evaluated TD-VD and just 62 deaths per 354100,000 population in May for TD-VD-CD. On the other hand, this study manifested REM for 355 women of 28 and 19 deaths per 100000 population in April and May respectively (Table 2 and 356 Fig. 75). We observed the same REM when we assessed TD-VD and just 15 deaths per 100,000 population in May for TD-VD-CD.

We did not find REM between January and March 2020 by age group (Table 3 and Figures 7678).Our results are compatible with REM in all the age group in April: 1,3,12,22 and 114 deaths per 1000,000 population for $0-11 y, 12-17 y, 18-29 y, 30-59 y$ and $\geq 60 y$ group severally (Table 3 and Fig. 79) .May REM was 56 and 619 deaths per 100000 population for 30-59y and $\geq 60 \mathrm{y}$ group respectively (Table 3 and Fig. 80). Note that when we assessed TD-VD, it did not represent a significant difference in REM. If we excluded TD-CD-CD, April REM was $1,3,10,12$ and 34114 deaths per 1000,000 population for the $0-11 \mathrm{y}, 12-17 \mathrm{y}, 18-29 \mathrm{y}, 30-59 \mathrm{y}$ and $\geq 60$ y group severally. May REM was 46 deaths per 100,000 population for the $30-59$ y group and 524 deaths per 100,000 population for $\geq 60 y$ group when we assessed TD-VD-CD.

\section{DISCUSSION}

370 Our findings illustrate 35 deaths in excess per 100000 population from all causes during April and May 2020 in Peru. Callao was the location with the highest excess mortality followed by

372 Lima, Ucayali, Loreto, Piura, Ica, Ancash, and La Libertad (119, 88, 81, 53, 40, 34, 22 and 11 373 deaths per 100000 population). Loreto was the only department with general excess mortality 
374 of 35 deaths in February 2020 and it could be explained for an outbreak of Dengue fever (23).

375 Furthermore, April excess mortality was observed for all age group in Ucayali, even excluding 397 clinical presentation $(30,31)$. causes. violent and COVID-19 deaths. That will require further investigation to establish the excess

Social distancing interventions has potential adverse outcomes on suicide and homicide risk is high(13,21). Although, suicide, homicide and motor vehicle deaths presented a sudden drop after the lockdown in Peru (22). We found that violent deaths did not mean a matter modification in REM in accordance with the above. Otherwise, the proportion of excess deaths that were attributed to COVID-19 increased over time.

Michelozzi and colleagues informed an excess mortality of 4,805 $(+45 \%)$ between the first week of March until 18 April in Italy(25). In the case of Peru, we only reported 1752 excess deaths $(+16.67 \%)$ in absolute numbers during April 2020. In England and Wales, the average of all-cause excess mortality was 79 per 100,000 population during 22 February to 08 May in 2020 while there was an excess of 35 Peruvian deaths per 100,000 population between 01 February and 31 May(26). The largest mortality ratios in European countries could be explained because the beginning of pandemic was earlier in February. Several researchers informed specific regions with increase in all-cause mortality in 2020 compared with historical mortality rate, especially in men and older people(27-29). Our data corroborate that national and locations excess was greater in men than women (55 and 15 deaths per 100000 population respectively). Differently, we found a national excess mortality of 35 deaths per 100000 population for 30-59 years group, but it was higher in individuals over 60 years (208 deaths per 100000 population). Besides, there was no excess of deaths among children and young people under 30 years which is consistent because this group is less likely to have a severe or critical 
Media has also assessed the excess mortality for Peru. The British Broadcasting Corporation

399 (BBC) estimated an excess of 87\% higher than average between 16 March to 31 May with about 18,900 more people dying than usual(38). Meanwhile, the Financial Times(FT) found an excess of 17,956 deaths from 04 March to 27 May (39). Unlike it, we did not obtain the estimation for the same periods of time; however, we obtained an excess of 10,201 deaths from 1 March to 31 May. The media data could be alarming and the result of an overestimation given that any death above the average of historical period has been considered an excess. Similar situations have happened in other countries which have estimated excess mortality $(29,40-42)$. Although, this raise could be expected.

One possible explanation for excess mortality is COVID-19 deaths without being diagnosed.

On the other hand, deaths related to other causes could represent an important excess cause because individuals postponed treatment to avoid the infection in private or public health centers or COVID-19 patients were prioritized or do not access.

A study showed 150,132 5-year prevalent cases which will need medical or surgical 412 management in the following years in Peru (33). Although, Peruvian health system has been 413 featured by inefficient performance, discontinuity and lack of assessment(34) and had one of 414 the lowest current health expenditure in Latin America and the Caribbean (35). Both conditions make it prone to not have suitable response in emergency situations. Even England and North Ireland informed significant falls of $45-66 \%$ in admissions for chemotherapy and $70-89 \%$ in 417 urgent referrals for early cancer diagnosis to pre-emergency level. It also found $78 \%$ of excess 418 deaths occur in cancer patients with $\geq 1$ comorbidity during COVID-19 pandemic(36). 419 Cardiovascular and Cancer excess deaths have been reported in Scotland and it may also 420 happened in Peru(37).

421 The limitations of this study are related with the fact that data is changing each moment because 422 of the epidemic is still growing. In addition, we have found a few missing data (sex, age) and 
some inconsistent information for Lambayeque. Even with these limitations, the findings

424 showed the most affected groups were male and individuals over 60 years old.

425 We consider that investigation of relevant excess mortality is a necessary, low cost and easy 426 method. It is important to compare the death toll of the first wave during pandemic and to 427 evaluate the effectiveness of policy responses and possible modifications. Additionally, 428 locations might differ in the distribution of its population, age, sex and comorbidities; so, some 429 places could be more vulnerable and need other kind of interventions. Therefore, we suggest to 430 expand its use to the 25 locations of Peru and to reanalyze responses due to $72 \%$ of Peruvian 431 people do not belong to the formal sector of the economy and they need going outside to 432 generate daily income(20) and it perpetuates transmission cycle and adverse outcomes.

\section{Conclusion}

435 Although, Peru was one of the first Latin American countries with the first restrictive measures, 436 COVID-19 deaths were significant and accounted for $45 \%$ of the relevant excess all-cause 437 deaths, leaving $54.29 \%$ unattributed to COVID-19. Hence, it is necessary to rethink the policy 438 measures in order to avoid the direct and indirect impact of COVID-19 and to find the suitable 439 way to reach population.

\section{$440 \quad$ List of Abbreviations}

441 - SARS-CoV-2: Severe acute respiratory syndrome coronavirus 2

442 - COVID-19: Coronavirus Disease 2019

443 - WHO: World Health Organization

444 - SINADEF: National Death Registry Information System

445 - NODP: National Open Data Platform

446 - EM: Excess mortality

447 - REM: Relevant Excess Mortality 
- TD: Total deaths

- TD-VD: Total deaths excluding violent deaths

- TD-VD-CD: Total deaths excluding violent and COVID-19 deaths

- BBC: British Broadcasting Corporation

- FT: Financial Times

\section{Declarations}

Ethics approval and consent to participate: The data used were anonymous from a public source so ethics approval was not required.

Consent for publication: Not applicable

Availability of data and materials: The data used in this study are freely available online in Spanish. http://www.minsa.gob.pe/reunis/data/defunciones_registradas.asp National Open Data Platform, https://www.datosabiertos.gob.pe/dataset/fallecidos-porcovid-19-ministerio-de-salud-minsa Funding: None Author contributions: JDMS and JEVR contributed to Conceptualization, Data curation, Formal analysis, Investigation, Methodology, Software, Visualization, Writing-original draft, Writing-review \& editing. All authors have read and approved the manuscript 


\section{TABLES Y FIGURES}

\section{Table 1: Relevant excess mortality for general absolute deaths and mortality rates}

\begin{tabular}{|c|c|c|c|c|c|c|c|c|c|c|c|c|c|c|c|c|c|c|c|}
\hline \multirow[b]{2}{*}{ Location } & \multirow[b]{2}{*}{ Month } & \multicolumn{3}{|c|}{$\begin{array}{c}\text { Limits and deviation } \\
\text { standard (DE) for absolute } \\
\text { deaths }\end{array}$} & \multicolumn{3}{|c|}{$\begin{array}{c}\begin{array}{c}\text { Limits and } \\
\text { deviation } \\
\text { standard (DE) } \\
\text { for mortality } \\
\text { rates }\end{array} \\
\end{array}$} & \multicolumn{4}{|c|}{ Total deaths (TD) } & \multicolumn{4}{|c|}{ TD - VD (Violent deaths) } & \multicolumn{4}{|c|}{ TD-VD-CD(COVID-19 Deaths) } \\
\hline & & $5 \% \mathrm{CI}$ & $95 \%$ CI & DE & $\begin{array}{r}5 \% \\
\text { CI }\end{array}$ & $\begin{array}{c}95 \% \\
\text { CI }\end{array}$ & DE & $\begin{array}{c}\text { Excess } \\
\text { deaths } \\
\text { from } \\
+2 D E \\
\text { above } \\
\text { upper } \\
\text { limit } \\
\end{array}$ & $\begin{array}{c}\text { Excess } \\
\text { deaths } \\
\text { from } \\
+2 \mathrm{DE} \\
\text { above } \\
\text { average }\end{array}$ & $\begin{array}{c}\text { Excess } \\
\text { mortality } \\
\text { rate from } \\
+2 D E \\
\text { above } \\
\text { upper } \\
\text { limit } \\
\end{array}$ & $\begin{array}{l}\text { Excess } \\
\text { mortality } \\
\text { rate from } \\
+2 \mathrm{DE} \\
\text { above } \\
\text { average }\end{array}$ & $\begin{array}{c}\text { Excess } \\
\text { deaths } \\
\text { from } \\
+2 D E \\
\text { above } \\
\text { upper } \\
\text { limit } \\
\end{array}$ & $\begin{array}{c}\text { Excess } \\
\text { deaths } \\
\text { from } \\
+2 \mathrm{DE} \\
\text { above } \\
\text { average }\end{array}$ & $\begin{array}{c}\text { Excess } \\
\text { mortality } \\
\text { rate from } \\
\text { +2DE } \\
\text { above } \\
\text { upper } \\
\text { limit } \\
\end{array}$ & $\begin{array}{l}\text { Excess } \\
\text { mortality } \\
\text { rate from } \\
+2 \mathrm{DE} \\
\text { above } \\
\text { average }\end{array}$ & 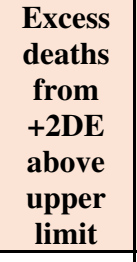 & $\begin{array}{c}\text { Excess } \\
\text { deaths } \\
\text { from } \\
+2 \mathrm{DE} \\
\text { above } \\
\text { average }\end{array}$ & $\begin{array}{c}\text { Excess } \\
\text { mortality } \\
\text { rate from } \\
\text { +2DE } \\
\text { above } \\
\text { upper } \\
\text { limit } \\
\end{array}$ & $\begin{array}{c}\text { Excess } \\
\text { mortality } \\
\text { rate from } \\
+2 \mathrm{DE} \\
\text { above } \\
\text { average }\end{array}$ \\
\hline \multirow{5}{*}{ Peru } & January & 7420.67 & 9801.33 & 1051.89 & 25 & 33 & 4 & -2279.11 & -1088.78 & -8 & -4 & -2474.11 & -1283.78 & -8 & -4 & -2474.11 & -1283.78 & -8 & -4 \\
\hline & February & 6966.91 & 9246.42 & 1007.20 & 24 & 31 & 3 & -2112.82 & -973.07 & -7 & -3 & -2314.82 & -1175.07 & -8 & -4 & -2314.82 & -1175.07 & -8 & -4 \\
\hline & March & 8109.96 & 9805.38 & 749.12 & 28 & 33 & 3 & -1943.62 & -1095.91 & -7 & -4 & -2049.62 & -1201.91 & -7 & -4 & -2115.62 & -1267.91 & -7 & -4 \\
\hline & April & 7495.14 & 9094.86 & 706.83 & 26 & 31 & 2 & 1751.48 & 2551.34 & 6 & 9 & 1713.48 & 2513.34 & 6 & 9 & -56.52 & 743.34 & 0 & 3 \\
\hline & May & 11530.01 & 13415.49 & 833.10 & 39 & 46 & 3 & 8449.31 & 9392.05 & 29 & 32 & 8389.31 & 9332.05 & 29 & 32 & 5587.31 & 6530.05 & 19 & 22 \\
\hline \multirow{5}{*}{ Lima } & January & 1911.43 & 2781.23 & 384.32 & 20 & 29 & 4 & -355.87 & 79.03 & -4 & 1 & -552.87 & -15.57 & -6 & -1 & -552.87 & -117.97 & -6 & -1 \\
\hline & February & 1788.14 & 2800.52 & 447.32 & 19 & 30 & 5 & -447.16 & 59.03 & -5 & 1 & -629.16 & -17.03 & -7 & -1 & -629.16 & -122.97 & -7 & -1 \\
\hline & March & 2387.44 & 2820.56 & 191.37 & 25 & 30 & 2 & 413.69 & 630.25 & 4 & 7 & 334.69 & 10.45 & 4 & 6 & 293.69 & 510.25 & 3 & 5 \\
\hline & April & 2074.19 & 2709.14 & 280.55 & 22 & 29 & 3 & 2333.75 & 2651.23 & 25 & 28 & 2304.75 & 70.48 & 24 & 28 & 1573.75 & 1891.23 & 17 & 20 \\
\hline & May & 4255.00 & 5049.00 & 350.83 & 45 & 53 & 4 & 5575.34 & 5972.34 & 59 & 63 & 5550.34 & 96.52 & 59 & 63 & 4536.34 & 4933.34 & 48 & 52 \\
\hline \multirow{5}{*}{ Lambeyeque } & January & 24 & 686 & 293 & 2 & 57 & 24 & -1229.96 & -898.86 & -103 & -75 & -1239.96 & -908.86 & -104 & -76 & -1239.96 & -908.86 & -104 & -76 \\
\hline & February & 51 & 643 & 261 & 4 & 54 & 22 & -1116.11 & -820.24 & -93 & -69 & -1125.11 & -829.24 & -94 & -69 & -1125.11 & -829.24 & -94 & -69 \\
\hline & March & 23 & 723 & 309 & 2 & 60 & 26 & -1296.19 & -945.97 & -108 & -79 & -1301.19 & -950.97 & -109 & -79 & -1307.19 & -956.97 & -109 & -80 \\
\hline & April & 60 & 589 & 234 & 5 & 49 & 20 & -784.00 & -519.25 & -65 & -43 & -788.00 & -523.25 & -66 & -44 & -1111.00 & -846.25 & -93 & -71 \\
\hline & May & 132 & 726 & 262 & 11 & 61 & 22 & -603.82 & -306.95 & -50 & -26 & -608.82 & -311.95 & -51 & -26 & -895.82 & -598.95 & -75 & -50 \\
\hline \multirow{2}{*}{ Piura } & January & 241 & 624 & 169 & 13 & 34 & 9 & -631 & -439 & -34 & -24 & -647 & -455 & -35 & -25 & -647 & -455 & -35 & -25 \\
\hline & February & 243 & 645 & 178 & 13 & 35 & 10 & -706 & -505 & -38 & -27 & -715 & -514 & -39 & -28 & -715 & -514 & -39 & -28 \\
\hline
\end{tabular}




\begin{tabular}{|c|c|c|c|c|c|c|c|c|c|c|c|c|c|c|c|c|c|c|c|}
\hline & March & 290 & 700 & 181 & 16 & 38 & 10 & -785 & -580 & -42 & -31 & -797 & -592 & -43 & -32 & -799 & -594 & -43 & -32 \\
\hline & April & 392 & 519 & 56 & 21 & 28 & 3 & -78 & -14 & -4 & -1 & -85 & -21 & -5 & -1 & -263 & -199 & -14 & -11 \\
\hline & May & 629 & 901 & 120 & 34 & 49 & 6 & 744 & 880 & 40 & 47 & 738 & 874 & 40 & 47 & 391 & 527 & 21 & 28 \\
\hline \multirow{5}{*}{ Loreto } & January & 180 & 198 & 8 & 20 & 22 & 1 & -16 & -7 & -2 & -1 & -33 & -24 & -4 & -3 & -33 & -24 & -4 & -3 \\
\hline & February & 140 & 145 & 2 & 16 & 16 & 0 & 35 & 37 & 4 & 4 & 28 & 30 & 3 & 3 & 28 & 30 & 3 & 3 \\
\hline & March & 137 & 186 & 22 & 16 & 21 & 2 & -47 & -23 & -5 & -3 & -49 & -25 & -6 & -3 & -51 & -27 & -6 & -3 \\
\hline & April & 126 & 192 & 29 & 14 & 22 & 3 & 75 & 108 & 8 & 12 & 73 & 106 & 8 & 12 & -37 & -4 & -4 & 0 \\
\hline & May & 329 & 427 & 43 & 37 & 48 & 5 & 401 & 450 & 45 & 51 & 398 & 447 & 45 & 51 & 213 & 262 & 24 & 30 \\
\hline \multirow{5}{*}{ Callao } & January & 343 & 367 & 10 & 35 & 37 & 1 & -49 & -37 & -5 & -4 & -68 & -56 & -7 & -6 & -68 & -56 & -7 & -6 \\
\hline & February & 270 & 391 & 54 & 27 & 39 & 5 & -121 & -60 & -12 & -6 & -146 & -85 & -15 & -9 & -146 & -85 & -15 & -9 \\
\hline & March & 322 & 366 & 20 & 32 & 37 & 2 & -22 & 1 & -2 & 0 & -30 & -7 & -3 & -1 & -34 & -11 & -3 & -1 \\
\hline & April & 286 & 346 & 26 & 29 & 35 & 3 & 244 & 273 & 25 & 27 & 242 & 271 & 24 & 27 & 118 & 147 & 12 & 15 \\
\hline & May & 680 & 715 & 15 & 68 & 72 & 2 & 932 & 949 & 94 & 95 & 925 & 942 & 93 & 95 & 724 & 741 & 73 & 75 \\
\hline \multirow{5}{*}{ Ancash } & January & 288 & 559 & 120 & 27 & 52 & 11 & -334 & -198 & -31 & -18 & -364 & -228 & -34 & -21 & -364 & -46 & -34 & -21 \\
\hline & February & 297 & 455 & 70 & 27 & 42 & 6 & -145 & -66 & -13 & -6 & -169 & -90 & -16 & -8 & -169 & -28 & -16 & -8 \\
\hline & March & 306 & 509 & 90 & 28 & 47 & 8 & -237 & -136 & -22 & -13 & -256 & -155 & -24 & -14 & -257 & -37 & -24 & -14 \\
\hline & April & 266 & 474 & 92 & 25 & 44 & 8 & -166 & -62 & -15 & -6 & -174 & -70 & -16 & -6 & -255 & -39 & -24 & -14 \\
\hline & May & 513 & 626 & 50 & 47 & 58 & 5 & 233 & 290 & 22 & 27 & 223 & 280 & 21 & 26 & 35 & 5 & 3 & 9 \\
\hline \multirow{5}{*}{ Ica } & January & 296 & 377 & 36 & 35 & 44 & 4 & -81 & -40 & -10 & -5 & -118 & -77 & -14 & -9 & -118 & -77 & -14 & -9 \\
\hline & February & 289 & 391 & 45 & 34 & 46 & 5 & -97 & -46 & -11 & -5 & -128 & -77 & -15 & -9 & -128 & -77 & -15 & -9 \\
\hline & March & 321 & 429 & 48 & 38 & 50 & 6 & -184 & -130 & -22 & -15 & -199 & -145 & -23 & -17 & -199 & -145 & -23 & -17 \\
\hline & April & 295 & 361 & 29 & 35 & 42 & 3 & -21 & 12 & -2 & 1 & -29 & 4 & -3 & 0 & -92 & -59 & -11 & -7 \\
\hline & May & 452 & 489 & 16 & 53 & 57 & 2 & 285 & 304 & 34 & 36 & 272 & 291 & 32 & 34 & 83 & 102 & 10 & 12 \\
\hline \multirow{4}{*}{ La Libertad } & January & 582 & 717 & 60 & 33 & 40 & 3 & -129 & -62 & -7 & -3 & -163 & -96 & -9 & -5 & -163 & -96 & -9 & -5 \\
\hline & February & 519 & 788 & 119 & 29 & 44 & 7 & -469 & -335 & -26 & -19 & -504 & -370 & -28 & -21 & -504 & -370 & -28 & -21 \\
\hline & March & 550 & 838 & 127 & 31 & 47 & 7 & -509 & -365 & -29 & -21 & -536 & -392 & -30 & -22 & -539 & -395 & -30 & -22 \\
\hline & April & 579 & 628 & 22 & 33 & 35 & 1 & -131 & -106 & -7 & -6 & -139 & -114 & -8 & -6 & -186 & -161 & -10 & -9 \\
\hline
\end{tabular}




\begin{tabular}{|c|c|c|c|c|c|c|c|c|c|c|c|c|c|c|c|c|c|c|c|}
\hline & May & 705 & 718 & 6 & 40 & 40 & 0 & 202 & 209 & 11 & 12 & 182 & 189 & 10 & 11 & 33 & 40 & 2 & 2 \\
\hline \multirow{5}{*}{ Ucayali } & January & 125 & 170 & 20 & 25 & 34 & 4 & -15 & 7 & -3 & 1 & -15 & 7 & -3 & 1 & -15 & 7 & -3 & 1 \\
\hline & February & 84 & 173 & 39 & 17 & 35 & 8 & -107 & -63 & -22 & -13 & -109 & -65 & -22 & -13 & -109 & -65 & -22 & -13 \\
\hline & March & 58 & 152 & 42 & 12 & 31 & 8 & -65 & -18 & -13 & -4 & -68 & -21 & -14 & -4 & -68 & -21 & -14 & -4 \\
\hline & April & 82 & 151 & 30 & 16 & 30 & 6 & 150 & 185 & 30 & 37 & 149 & 184 & 30 & 37 & 100 & 135 & 20 & 27 \\
\hline & May & 219 & 347 & 57 & 44 & 70 & 11 & 255 & 319 & 51 & 64 & 255 & 319 & 51 & 64 & 198 & 262 & 40 & 53 \\
\hline
\end{tabular}

TD: Total deaths, VD: Violent deaths, CV: COVID-19 deaths

Table 2: Relevant excess mortality for general absolute deaths and mortality rates by sex

\begin{tabular}{|c|c|c|c|c|c|c|c|c|c|c|c|c|c|c|c|c|c|c|c|c|}
\hline \multirow[b]{2}{*}{ Location } & \multirow[b]{2}{*}{ Sex } & \multirow[b]{2}{*}{ Month } & \multicolumn{3}{|c|}{$\begin{array}{l}\text { Limits and deviation } \\
\text { standard(DE) for } \\
\text { absolute deaths }\end{array}$} & \multicolumn{3}{|c|}{$\begin{array}{c}\text { Limits and } \\
\text { deviation } \\
\text { standard(DE) } \\
\text { for mortality } \\
\text { rates } \\
\end{array}$} & \multicolumn{4}{|c|}{$\mathrm{TD}$} & \multicolumn{4}{|c|}{ TD - VD } & \multicolumn{4}{|c|}{ TD-VD-CD } \\
\hline & & & $5 \% \mathrm{CI}$ & $95 \% \mathrm{CI}$ & DE & $\begin{array}{l}5 \% \\
\text { CI }\end{array}$ & $\begin{array}{c}95 \% \\
\text { CI }\end{array}$ & DE & 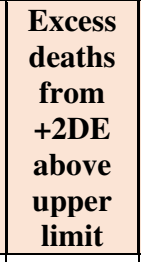 & $\begin{array}{c}\text { Excess } \\
\text { deaths } \\
\text { from } \\
\text { +2DE } \\
\text { above } \\
\text { average } \\
\end{array}$ & \begin{tabular}{|c|} 
Excess \\
mortality \\
rate from \\
$+2 D E$ \\
above \\
upper \\
limit \\
\end{tabular} & $\begin{array}{c}\text { Excess } \\
\text { mortality } \\
\text { rate from } \\
\text { +2DE } \\
\text { above } \\
\text { average }\end{array}$ & 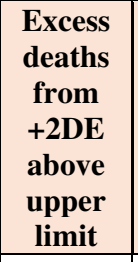 & $\begin{array}{c}\text { Excess } \\
\text { deaths } \\
\text { from } \\
+2 \mathrm{DE} \\
\text { above } \\
\text { average }\end{array}$ & \begin{tabular}{|c|} 
Excess \\
mortality \\
rate from \\
$+2 D E$ \\
above \\
upper \\
limit \\
\end{tabular} & $\begin{array}{c}\text { Excess } \\
\text { mortality } \\
\text { rate from } \\
+2 \mathrm{DE} \\
\text { above } \\
\text { average }\end{array}$ & 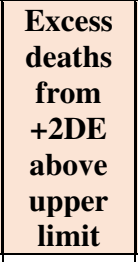 & \begin{tabular}{|} 
Excess \\
deaths \\
from \\
$+2 \mathrm{DE}$ \\
above \\
average \\
\end{tabular} & \begin{tabular}{|c} 
Excess \\
mortality \\
rate from \\
$+2 D E$ \\
above \\
upper \\
limit \\
\end{tabular} & $\begin{array}{c}\text { Excess } \\
\text { mortality } \\
\text { rate from } \\
+2 D E \\
\text { above } \\
\text { average }\end{array}$ \\
\hline \multirow{10}{*}{ Peru } & \multirow{5}{*}{ Male } & January & 3,913 & 5,193 & 565 & 27 & 36 & 4 & -1276.19 & -636.29 & -9 & -4 & -1780.19 & -1140.29 & -12 & -8 & -1780.19 & -1140.29 & -12 & -8 \\
\hline & & February & 3,73 & 4,9 & 517 & 26 & 34 & 4 & -1095.57 & -510.34 & -8 & -4 & -1591.57 & -1006.34 & -11 & -7 & -1591.57 & -1006.34 & -11 & -7 \\
\hline & & March & 4,305 & 5,21 & 400 & 30 & 36 & 3 & -1064.48 & -612.01 & -7 & -4 & -1379.48 & -927.01 & -10 & -6 & -1429.48 & -977.01 & -10 & -7 \\
\hline & & April & 4,049 & 4,841 & 350 & 28 & 34 & 2 & 1606.08 & 2002.45 & 11 & 14 & 1488.08 & 1884.45 & 10 & 13 & 206.08 & 602.45 & 1 & 4 \\
\hline & & May & 6,825 & 7,702 & 387 & 47 & 53 & 3 & 6405.30 & 6843.33 & 44 & 47 & 6270.30 & 6708.33 & 43 & 46 & 4541.30 & 4979.33 & 31 & 34 \\
\hline & \multirow{5}{*}{ Female } & January & 3492.94 & 4621.06 & 498.46 & 23 & 31 & 3 & -1035.98 & -471.92 & -7 & -3 & -1223.98 & -659.92 & -8 & -4 & -1223.98 & -659.92 & -8 & -4 \\
\hline & & February & 3224.29 & 4357.04 & 500.50 & 22 & 29 & 3 & -1036.05 & -469.67 & -7 & -3 & -1179.05 & -612.67 & -8 & -4 & -1179.05 & -612.67 & -8 & -4 \\
\hline & & March & 3799.69 & 4600.98 & 354.05 & 25 & 31 & 2 & -875.07 & -474.43 & -6 & -3 & -957.07 & -556.43 & -6 & -4 & \begin{tabular}{|l|}
-973.07 \\
\end{tabular} & -572.43 & -7 & -4 \\
\hline & & April & 3442.55 & 4251.45 & 357.41 & 23 & 28 & 2 & 172.74 & 577.18 & 1 & 4 & 121.74 & 526.18 & 1 & 4 & -386.26 & 18.18 & -3 & 0 \\
\hline & & May & 4713.52 & 5723.48 & 446.25 & 32 & 38 & 3 & 2075.01 & 2580.00 & 14 & 17 & 2028.01 & 2533.00 & 14 & 17 & 1363.01 & 1868.00 & 9 & 13 \\
\hline
\end{tabular}




\begin{tabular}{|c|c|c|c|c|c|c|c|c|c|c|c|c|c|c|c|c|c|c|c|c|}
\hline \multirow{10}{*}{ Lima } & \multirow{5}{*}{ Male } & January & 1022.44 & 1462.23 & 194.32 & 22 & 32 & 4 & -260.88 & -40.98 & -6 & -1 & -400.88 & -180.98 & -9 & -4 & -400.88 & -180.98 & -9 & -4 \\
\hline & & February & 954.05 & 1491.28 & 237.37 & 21 & 32 & 5 & -239.03 & 29.59 & -5 & 1 & -391.03 & -122.41 & -8 & -3 & -391.03 & -122.41 & -8 & -3 \\
\hline & & March & 1271.67 & 1470.99 & 88.07 & 27 & 32 & 2 & 215.87 & 315.53 & 5 & 7 & 155.87 & 255.53 & 3 & 6 & 124.87 & 224.53 & 3 & 5 \\
\hline & & April & 1116.97 & 1433.70 & 139.95 & 24 & 31 & 3 & 1605.41 & 1763.77 & 35 & 38 & 1585.41 & 1743.77 & 34 & 38 & 1057.41 & 1215.77 & 23 & 26 \\
\hline & & May & 2613.87 & 3000.13 & 170.67 & 57 & 65 & 4 & 3987.52 & 4180.65 & 86 & 90 & 3966.52 & 4159.65 & 86 & 90 & 3386.52 & 3579.65 & 73 & 77 \\
\hline & \multirow{5}{*}{ Female } & January & 882 & 1,323 & 195 & 18 & 27 & 4 & -110.16 & 110.44 & -2 & 2 & -167.16 & 53.44 & -3 & 1 & -167.16 & 53.44 & -3 & 1 \\
\hline & & February & 827 & 1,314 & 215 & 17 & 27 & 4 & -220.81 & 22.43 & -5 & 0 & -252.81 & -9.57 & -5 & 0 & -252.81 & -9.57 & -5 & 0 \\
\hline & & March & 1,109 & 1,351 & 107 & 23 & 28 & 2 & 190.48 & 311.48 & 4 & 6 & 171.48 & 292.48 & 4 & 6 & 161.48 & 282.48 & 3 & 6 \\
\hline & & April & 955 & 1,273 & 141 & 20 & 26 & 3 & 749.77 & 909.21 & 15 & 19 & 740.77 & 900.21 & 15 & 19 & 537.77 & 697.21 & 11 & 14 \\
\hline & & May & 1,636 & 2,05 & 183 & 34 & 42 & 4 & 1576.97 & 1783.67 & 32 & 37 & 1572.97 & 1779.67 & 32 & 37 & 1377.97 & 1584.67 & 28 & 33 \\
\hline \multirow{10}{*}{ Lambayeque } & \multirow{5}{*}{ Male } & January & 14 & 328 & 139 & 2 & 56 & 24 & -579 & -422 & -100 & -73 & -586 & -429 & -101 & -74 & -586 & -429 & -101 & -74 \\
\hline & & February & 31 & 317 & 127 & 5 & 55 & 22 & -544 & -401 & -94 & -69 & -552 & -409 & -95 & -70 & -552 & -409 & -95 & -70 \\
\hline & & March & 5 & 377 & 165 & 1 & 65 & 28 & -677 & -491 & -117 & -85 & -682 & -496 & -117 & -85 & -686 & -500 & -118 & -86 \\
\hline & & April & 35 & 318 & 125 & 6 & 55 & 22 & -382 & -241 & -66 & -41 & -386 & -245 & -66 & -42 & -599 & -458 & -103 & -79 \\
\hline & & May & 105 & 404 & 132 & 18 & 70 & 23 & -206 & -57 & -35 & -10 & -209 & -60 & -36 & -10 & -407 & -258 & -70 & -44 \\
\hline & \multirow{5}{*}{ Female } & January & 9 & 357 & 154 & 2 & 58 & 25 & -650 & -476 & -105 & -77 & -653 & -479 & -106 & -78 & -653 & -479 & -106 & -78 \\
\hline & & February & 20 & 327 & 135 & 3 & 53 & 22 & -574 & -421 & -93 & -68 & -575 & -422 & -93 & -68 & -575 & -422 & -93 & -68 \\
\hline & & March & 17 & 347 & 145 & 3 & 56 & 24 & -620 & -456 & -101 & -74 & -620 & -456 & -101 & -74 & -622 & -458 & -101 & -74 \\
\hline & & April & 24 & 272 & 110 & 4 & 44 & 18 & -404 & -280 & -66 & -45 & -404 & -280 & -66 & -45 & -514 & -390 & -83 & -63 \\
\hline & & May & 26 & 323 & 131 & 4 & 52 & 21 & -401 & -252 & -65 & -41 & -402 & -253 & -65 & -41 & -483 & -334 & -78 & -54 \\
\hline \multirow{7}{*}{ Piura } & \multirow{5}{*}{ Male } & January & 126 & 337 & 93 & 14 & 37 & 10 & -331 & -225 & -36 & -25 & -343 & -237 & -37 & -26 & -343 & -237 & -37 & -26 \\
\hline & & February & 134 & 362 & 101 & 15 & 39 & 11 & -404 & -290 & -44 & -32 & -410 & -296 & -45 & -32 & -410 & -296 & -45 & -32 \\
\hline & & March & 153 & 399 & 109 & 17 & 43 & 12 & -483 & -360 & -53 & -39 & -494 & -371 & -54 & -40 & -496 & -373 & -54 & -41 \\
\hline & & April & 201 & 269 & 30 & 22 & 29 & 3 & 23 & 57 & 3 & 6 & 17 & 51 & 2 & 6 & -110 & -76 & -12 & -8 \\
\hline & & May & 397 & 552 & 69 & 43 & 60 & 7 & 572 & 649 & 62 & 71 & 567 & 644 & 62 & 70 & 344 & 421 & 37 & 46 \\
\hline & \multirow{2}{*}{ Female } & January & 112 & 290 & 79 & 12 & 31 & 8 & -308 & -219 & -33 & -23 & -312 & -223 & -33 & -24 & -312 & -223 & -33 & -24 \\
\hline & & February & 108 & 284 & 77 & 12 & 30 & 8 & -303 & -216 & -32 & -23 & -306 & -219 & -33 & -23 & -306 & -219 & -33 & -23 \\
\hline
\end{tabular}




\begin{tabular}{|c|c|c|c|c|c|c|c|c|c|c|c|c|c|c|c|c|c|c|c|c|}
\hline & & March & 136 & 300 & 73 & 15 & 32 & 8 & -298 & -216 & -32 & -23 & -299 & -217 & -32 & -23 & -299 & -217 & -32 & -23 \\
\hline & & April & 189 & 251 & 28 & 20 & 27 & 3 & -97 & -66 & -10 & -7 & -98 & -67 & -10 & -7 & -149 & -118 & -16 & -13 \\
\hline & & May & 233 & 349 & 51 & 25 & 37 & 5 & 172 & 230 & 18 & 25 & 172 & 230 & 18 & 25 & 68 & 126 & 7 & 13 \\
\hline \multirow{10}{*}{ Loreto } & \multirow{5}{*}{ Male } & January & 102 & 115 & 6 & 23 & 26 & 1 & -20 & -14 & -4 & -3 & -24 & -18 & -5 & -4 & -24 & -18 & -5 & -4 \\
\hline & & February & 71 & 83 & 5 & 16 & 19 & 1 & 7 & 13 & 2 & 3 & 2 & 8 & 1 & 2 & 2 & 8 & 1 & 2 \\
\hline & & March & 78 & 108 & 13 & 17 & 24 & 3 & -31 & -16 & -7 & -4 & -32 & -17 & -7 & -4 & -34 & -19 & -8 & -4 \\
\hline & & April & 67 & 119 & 23 & 15 & 27 & 5 & 65 & 91 & 15 & 20 & 64 & 90 & 14 & 20 & -17 & 9 & -4 & 2 \\
\hline & & May & 207 & 277 & 31 & 47 & 62 & 7 & 286 & 321 & 64 & 72 & 283 & 318 & 64 & 72 & 151 & 186 & 34 & 42 \\
\hline & \multirow{5}{*}{ Female } & January & 68 & 91 & 10 & 15 & 21 & 2 & -29 & -18 & -7 & -4 & -30 & -19 & -7 & -4 & -30 & -19 & -7 & -4 \\
\hline & & February & 59 & 72 & 6 & 13 & 16 & 1 & 1 & 8 & 0 & 2 & -1 & 6 & 0 & 1 & -1 & 6 & 0 & 1 \\
\hline & & March & 46 & 92 & 21 & 10 & 21 & 5 & -52 & -28 & -12 & -6 & -53 & -29 & -12 & -7 & -53 & -29 & -12 & -7 \\
\hline & & April & 56 & 74 & 8 & 13 & 17 & 2 & 24 & 33 & 5 & 8 & 23 & 32 & 5 & 7 & -6 & 3 & -1 & 1 \\
\hline & & May & 122 & 151 & 13 & 28 & 34 & 3 & 113 & 128 & 26 & 29 & 113 & 128 & 26 & 29 & 64 & 79 & 15 & 18 \\
\hline \multirow{10}{*}{ Callao } & \multirow{5}{*}{ Male } & January & 192 & 198 & 3 & 40 & 41 & 1 & -15 & -12 & -3 & -3 & -33 & -30 & -7 & -6 & -33 & -30 & -7 & -6 \\
\hline & & February & 155 & 202 & 21 & 32 & 41 & 4 & -49 & -25 & -10 & -5 & -70 & -46 & -14 & -10 & -70 & -46 & -14 & -10 \\
\hline & & March & 178 & 210 & 14 & 37 & 43 & 3 & -22 & -6 & -5 & -1 & -27 & -11 & -6 & -2 & -29 & -13 & -6 & -3 \\
\hline & & April & 164 & 193 & 13 & 34 & 40 & 3 & 187 & 202 & 39 & 42 & 185 & 200 & 38 & 41 & 92 & 107 & 19 & 22 \\
\hline & & May & 420 & 435 & 7 & 87 & 90 & 1 & 618 & 626 & 127 & 129 & 613 & 621 & 126 & 128 & 495 & 503 & 102 & 104 \\
\hline & \multirow{5}{*}{ Female } & January & 151 & 169 & 8 & 30 & 33 & 2 & -35 & -26 & -7 & -5 & -35 & -26 & -7 & -5 & -35 & -26 & -7 & -5 \\
\hline & & February & 115 & 190 & 33 & 23 & 37 & 7 & -72 & -35 & -14 & -7 & -76 & -39 & -15 & -8 & -76 & -39 & -15 & -8 \\
\hline & & March & 147 & 177 & 13 & 29 & 35 & 3 & -34 & -19 & -7 & -4 & -36 & -21 & -7 & -4 & -38 & -23 & -7 & -5 \\
\hline & & April & 137 & 168 & 13 & 27 & 33 & 3 & 42 & 57 & 8 & 11 & 42 & 57 & 8 & 11 & 11 & 26 & 2 & 5 \\
\hline & & May & 256 & 284 & 12 & 50 & 56 & 2 & 304 & 317 & 60 & 62 & 304 & 317 & 60 & 62 & 252 & 265 & 49 & 52 \\
\hline \multirow{4}{*}{ Ancash } & \multirow{4}{*}{ Male } & January & 145 & 278 & 59 & 27 & 52 & 11 & -146 & -80 & -27 & -15 & -166 & -100 & -31 & -19 & -166 & -100 & -31 & -19 \\
\hline & & February & 171 & 239 & 30 & 32 & 45 & 6 & -59 & -25 & -11 & -5 & -77 & -43 & -14 & -8 & -77 & -43 & -14 & -8 \\
\hline & & March & 171 & 262 & 40 & 32 & 49 & 7 & -91 & -46 & -17 & -9 & -104 & -59 & -19 & -11 & -104 & -59 & -19 & -11 \\
\hline & & April & 124 & 246 & 54 & 23 & 46 & 10 & -83 & -22 & -16 & -4 & -88 & -27 & -17 & -5 & -146 & -85 & -27 & -16 \\
\hline
\end{tabular}




\begin{tabular}{|c|c|c|c|c|c|c|c|c|c|c|c|c|c|c|c|c|c|c|c|c|}
\hline & & May & 306 & 339 & 15 & 57 & 63 & 3 & 245 & 261 & 46 & 49 & 236 & 252 & 44 & 47 & 112 & 128 & 21 & 24 \\
\hline & & January & 140 & 282 & 62 & 26 & 51 & 11 & -187 & -117 & -34 & -21 & -197 & -127 & -36 & -23 & -197 & -127 & -36 & -23 \\
\hline & & February & 123 & 219 & 43 & 22 & 40 & 8 & -83 & -35 & -15 & -6 & -89 & -41 & -16 & -7 & -89 & -41 & -16 & -7 \\
\hline & Female & March & 137 & 247 & 49 & 25 & 45 & 9 & -131 & -76 & -24 & -14 & -137 & -82 & -25 & -15 & -138 & -83 & -25 & -15 \\
\hline & & April & 142 & 229 & 39 & 26 & 42 & 7 & -71 & -28 & -13 & -5 & -74 & -31 & -14 & -6 & -97 & -54 & -18 & -10 \\
\hline & & May & 199 & 294 & 42 & 36 & 54 & 8 & -32 & 16 & -6 & 3 & -33 & 15 & -6 & 3 & -79 & -31 & -14 & -6 \\
\hline \multirow{10}{*}{ Ica } & \multirow{5}{*}{ Male } & January & 157 & 183 & 12 & 37 & 44 & 3 & -2 & 11 & 0 & 3 & -17 & -4 & -4 & -1 & -17 & -4 & -4 & -1 \\
\hline & & February & 163 & 198 & 16 & 39 & 47 & 4 & -26 & -9 & -6 & -2 & -48 & -31 & -12 & -7 & -48 & -31 & -12 & -7 \\
\hline & & March & 174 & 206 & 14 & 42 & 49 & 3 & -52 & -36 & -12 & -9 & -63 & -47 & -15 & -11 & -63 & -47 & -15 & -11 \\
\hline & & April & 175 & 180 & 2 & 42 & 43 & 0 & 50 & 53 & 12 & 13 & 43 & 46 & 10 & 11 & 0 & 3 & 0 & 1 \\
\hline & & May & 254 & 271 & 8 & 60 & 65 & 2 & 203 & 212 & 48 & 50 & 191 & 200 & 46 & 48 & 85 & 94 & 20 & 22 \\
\hline & \multirow{5}{*}{ Female } & January & 137 & 194 & 25 & 32 & 45 & 6 & -82 & -53 & -19 & -12 & -104 & -75 & -24 & -17 & -104 & -75 & -24 & -17 \\
\hline & & February & 128 & 197 & 30 & 30 & 46 & 7 & -77 & -42 & -18 & -10 & -86 & -51 & -20 & -12 & -86 & -51 & -20 & -12 \\
\hline & & March & 138 & 224 & 38 & 32 & 52 & 9 & -141 & -98 & -33 & -23 & -145 & -102 & -34 & -24 & -145 & -102 & -34 & -24 \\
\hline & & April & 132 & 179 & 21 & 31 & 42 & 5 & -56 & -33 & -13 & -8 & -57 & -34 & -13 & -8 & -77 & -54 & -18 & -12 \\
\hline & & May & 185 & 223 & 17 & 43 & 52 & 4 & 60 & 80 & 14 & 19 & 59 & 79 & 14 & 18 & 0 & 20 & 0 & 5 \\
\hline \multirow{10}{*}{ La Libertad } & \multirow{5}{*}{ Male } & January & 300 & 392 & 41 & 35 & 45 & 5 & -106 & -60 & -12 & -7 & -143 & -97 & -16 & -11 & -143 & -97 & -16 & -11 \\
\hline & & February & 288 & 405 & 52 & 33 & 47 & 6 & -218 & -159 & -25 & -18 & -246 & -187 & -28 & -22 & -246 & -187 & -28 & -22 \\
\hline & & March & 276 & 422 & 64 & 32 & 49 & 7 & -250 & -177 & -29 & -20 & -274 & -201 & -32 & -23 & -276 & -203 & -32 & -23 \\
\hline & & April & 290 & 356 & 29 & 33 & 41 & 3 & -130 & -97 & -15 & -11 & -135 & -102 & -16 & -12 & -166 & -133 & -19 & -15 \\
\hline & & May & 381 & 393 & 6 & 44 & 45 & 1 & 149 & 155 & 17 & 18 & 135 & 141 & 16 & 16 & 31 & 37 & 4 & 4 \\
\hline & \multirow{5}{*}{ Female } & January & 270 & 337 & 29 & 30 & 37 & 3 & -57 & -23 & -6 & -3 & -63 & -29 & -7 & -3 & -63 & -29 & -7 & -3 \\
\hline & & February & 232 & 362 & 58 & 26 & 40 & 6 & -212 & -147 & -23 & -16 & -219 & -154 & -24 & -17 & -219 & -154 & -24 & -17 \\
\hline & & March & 270 & 419 & 66 & 30 & 46 & 7 & -269 & -195 & -30 & -21 & -272 & -198 & -30 & -22 & -273 & -199 & -30 & -22 \\
\hline & & April & 271 & 289 & 8 & 30 & 32 & 1 & -51 & -41 & -6 & -5 & -54 & -44 & -6 & -5 & -70 & -60 & -8 & -7 \\
\hline & & May & 314 & 335 & 9 & 35 & 37 & 1 & 25 & 36 & 3 & 4 & 19 & 30 & 2 & 3 & -6 & 5 & -1 & 1 \\
\hline Ucayali & Male & January & 69 & 95 & 11 & 28 & 38 & 4 & -6 & 7 & -2 & 3 & -7 & 6 & -3 & 2 & -7 & 6 & -3 & 2 \\
\hline
\end{tabular}




\begin{tabular}{|c|c|c|c|c|c|c|c|c|c|c|c|c|c|c|c|c|c|c|c|}
\hline & February & 53 & 92 & 17 & 21 & 37 & 7 & -49 & -29 & -19 & -12 & -51 & -31 & -20 & -13 & -51 & -31 & -20 & -13 \\
\hline & March & 36 & 86 & 22 & 15 & 34 & 9 & -43 & -18 & -17 & -7 & -46 & -21 & -19 & -9 & -46 & -21 & -19 & -9 \\
\hline & April & 43 & 94 & 23 & 17 & 38 & 9 & -118 & -92 & -47 & -37 & -119 & -93 & -47 & -37 & -156 & -130 & -62 & -52 \\
\hline & May & 145 & 216 & 32 & 58 & 86 & 13 & 200 & 235 & 80 & 94 & 200 & 235 & 80 & 94 & 155 & 190 & 62 & 76 \\
\hline \multirow{5}{*}{ Female } & January & 53 & 79 & 11 & 21 & 32 & 5 & -17 & -4 & -7 & -2 & -17 & -4 & -7 & -2 & -17 & -4 & -7 & -2 \\
\hline & February & 27 & 86 & 26 & 11 & 35 & 10 & -70 & -41 & -28 & -17 & -71 & -42 & -29 & -17 & -71 & -42 & -29 & -17 \\
\hline & March & 21 & 67 & 20 & 9 & 27 & 8 & -25 & -2 & -10 & -1 & -25 & -2 & -10 & -1 & -25 & -2 & -10 & -1 \\
\hline & April & 39 & 56 & 8 & 16 & 23 & 3 & 68 & 77 & 28 & 31 & 68 & 77 & 28 & 31 & 56 & 65 & 23 & 26 \\
\hline & May & 71 & 128 & 25 & 29 & 52 & 10 & 48 & 76 & 19 & 31 & 48 & 76 & 19 & 31 & 37 & 65 & 15 & 27 \\
\hline
\end{tabular}

TD: Total deaths, VD: Violent deaths, CV: COVID-19 deaths

79 Table 3: Relevant excess mortality for general absolute deaths and mortality rates by age group

\begin{tabular}{|c|c|c|c|c|c|c|c|c|c|c|c|c|c|c|c|c|c|c|c|c|}
\hline \multirow[b]{2}{*}{ Location } & \multirow[b]{2}{*}{$\begin{array}{c}\text { Age } \\
\text { group }\end{array}$} & \multirow[b]{2}{*}{ Month } & \multicolumn{3}{|c|}{$\begin{array}{l}\text { Limits and deviation } \\
\text { standard(DE) for } \\
\text { absolute deaths }\end{array}$} & \multicolumn{3}{|c|}{$\begin{array}{c}\text { Limits and } \\
\text { deviation } \\
\text { standard(DE) } \\
\text { for mortality } \\
\text { rates } \\
\end{array}$} & \multicolumn{4}{|c|}{ TD } & \multicolumn{4}{|c|}{ TD - VD } & \multicolumn{4}{|c|}{ TD-VD-CD } \\
\hline & & & $5 \% \mathrm{CI}$ & $95 \% \mathrm{CI}$ & DE & $\begin{array}{l}5 \% \\
\text { CI }\end{array}$ & $\begin{array}{c}95 \% \\
\text { CI }\end{array}$ & DE & $\begin{array}{c}\text { Excess } \\
\text { deaths } \\
\text { from } \\
+2 \mathrm{DE} \\
\text { above } \\
\text { upper } \\
\text { limit } \\
\end{array}$ & $\begin{array}{c}\text { Excess } \\
\text { deaths } \\
\text { from } \\
+2 \mathrm{DE} \\
\text { above } \\
\text { average } \\
\end{array}$ & $\begin{array}{c}\text { Excess } \\
\text { mortality } \\
\text { rate from } \\
+2 D E \\
\text { above } \\
\text { upper } \\
\text { limit } \\
\end{array}$ & $\begin{array}{c}\text { Excess } \\
\text { mortality } \\
\text { rate from } \\
+2 \mathrm{DE} \\
\text { above } \\
\text { average } \\
\end{array}$ & 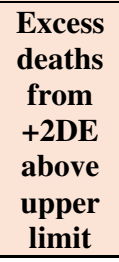 & $\begin{array}{c}\text { Excess } \\
\text { deaths } \\
\text { from } \\
+2 D E \\
\text { above } \\
\text { average } \\
\end{array}$ & $\begin{array}{c}\text { Excess } \\
\text { mortality } \\
\text { rate from } \\
+2 \mathrm{DE} \\
\text { above } \\
\text { upper } \\
\text { limit } \\
\end{array}$ & $\begin{array}{c}\text { Excess } \\
\text { mortality } \\
\text { rate from } \\
+2 \mathrm{DE} \\
\text { above } \\
\text { average } \\
\end{array}$ & $\begin{array}{c}\text { Excess } \\
\text { deaths } \\
\text { from } \\
+2 D E \\
\text { above } \\
\text { upper } \\
\text { limit } \\
\end{array}$ & $\begin{array}{c}\text { Excess } \\
\text { deaths } \\
\text { from } \\
+2 \mathrm{DE} \\
\text { above } \\
\text { average }\end{array}$ & $\begin{array}{c}\text { Excess } \\
\text { mortality } \\
\text { rate from } \\
+2 D E \\
\text { above } \\
\text { upper } \\
\text { limit } \\
\end{array}$ & $\begin{array}{c}\text { Excess } \\
\text { mortality } \\
\text { rate from } \\
+2 \mathrm{DE} \\
\text { above } \\
\text { average }\end{array}$ \\
\hline \multirow{7}{*}{ Peru } & \multirow{5}{*}{$0-11 y$} & January & 472 & 617 & 64 & 8 & 10 & 1 & -151.61 & -79.36 & -2 & -1 & -202.61 & -130.36 & -3 & -2 & -202.61 & -130.36 & -3 & -2 \\
\hline & & February & 457 & 555 & 43 & 7 & 9 & 1 & -74 & -25 & -1 & 0 & -126 & -77 & -2 & -1 & -126 & -77 & -2 & -1 \\
\hline & & March & 518 & 603 & 37 & 8 & 10 & 1 & -222 & -180 & -4 & -3 & -261 & -219 & -4 & -4 & -261 & -219 & -4 & -4 \\
\hline & & April & 497 & 573 & 33 & 8 & 9 & 1 & -267 & -229 & -4 & -4 & -288 & -250 & -5 & -4 & -294 & -256 & -5 & -4 \\
\hline & & May & 541 & 603 & 27 & 9 & 10 & 0 & -286 & -255 & -5 & -4 & -306 & -275 & -5 & -4 & -319 & -288 & -5 & -5 \\
\hline & \multirow{2}{*}{$12-17 y$} & January & 82 & 108 & 12 & 3 & 4 & 0 & -34.12 & -21.07 & -1 & -1 & -63.12 & -50.07 & -2 & -2 & -63.12 & -50.07 & -2 & -2 \\
\hline & & February & 82 & 121 & 17 & 3 & 4 & 1 & -50 & -30 & -2 & -1 & -86 & -66 & -3 & -2 & -86 & -66 & -3 & -2 \\
\hline
\end{tabular}




\begin{tabular}{|c|c|c|c|c|c|c|c|c|c|c|c|c|c|c|c|c|c|c|c|c|}
\hline & & March & 90 & 99 & 4 & 3 & 3 & 0 & -29 & -25 & -1 & -1 & -50 & -46 & -2 & -2 & -50 & -46 & -2 & -2 \\
\hline & & April & 80 & 84 & 2 & 3 & 3 & 0 & -8 & -6 & 0 & 0 & -22 & -20 & -1 & -1 & -25 & -23 & -1 & -1 \\
\hline & & May & 81 & 110 & 13 & 3 & 4 & 0 & -56 & -41 & -2 & -1 & -76 & -61 & -3 & -2 & -78 & -63 & -3 & -2 \\
\hline & & January & 351 & 415 & 28 & 6 & 7 & 0 & -27.80 & 4.29 & 0 & 0 & -223.80 & -191.71 & -4 & -3 & -223.80 & \begin{tabular}{|l}
-191.71 \\
\end{tabular} & -4 & -3 \\
\hline & & February & 324 & 404 & 35 & 6 & 7 & 1 & -93 & -53 & -2 & -1 & -218 & -178 & -4 & -3 & -218 & -178 & -4 & -3 \\
\hline & $18-29 y$ & March & 324 & 428 & 46 & 6 & 7 & 1 & -225 & -173 & -4 & -3 & -294 & -242 & -5 & -4 & -296 & -244 & -5 & -4 \\
\hline & & April & 309 & 397 & 39 & 5 & 7 & 1 & -186 & -142 & -3 & -2 & -212 & -168 & -4 & -3 & -231 & -187 & -4 & -3 \\
\hline & & May & 328 & 427 & 44 & 6 & 7 & 1 & -143 & -94 & -2 & -2 & -174 & -125 & -3 & -2 & -192 & -143 & -3 & -2 \\
\hline & & January & 1548.16 & 1990.51 & 195.45 & 14 & 18 & 2 & -398.42 & -177.24 & -4 & -2 & -699.42 & -478.24 & -6 & -4 & -699.42 & -478.24 & -6 & -4 \\
\hline & & February & 1,453 & 1,823 & 163 & 13 & 17 & 2 & -365 & -180 & -3 & -2 & -637 & -452 & -6 & -4 & -637 & -452 & -6 & -4 \\
\hline & $30-59 y$ & March & 1,554 & 1,964 & 181 & 14 & 18 & 2 & -590 & -385 & -5 & -4 & -770 & -565 & -7 & -5 & -790 & -585 & -7 & -5 \\
\hline & & April & 1,504 & 1,838 & 148 & 14 & 17 & 1 & 560 & 727 & 5 & 7 & 491 & 658 & 5 & 6 & -63 & 104 & -1 & 1 \\
\hline & & May & 1,629 & 1,979 & 154 & 15 & 18 & 1 & 3,191 & 3,365 & 30 & 31 & 3,145 & 3,319 & 29 & 31 & 2,328 & 2,502 & 22 & 23 \\
\hline & & January & 5115.41 & 6849.59 & 766.24 & 146 & 196 & 22 & -1873.08 & -1005.99 & -54 & -29 & -1987.08 & -1119.99 & -57 & -32 & -1987.08 & -1119.99 & -57 & -32 \\
\hline & & February & 4,843 & 6,554 & 756 & 138 & 187 & 22 & $-1,744$ & -888 & -50 & -25 & $-1,898$ & $-1,042$ & -54 & -30 & $-1,898$ & $-1,042$ & -54 & -30 \\
\hline & $\geq 60 y$ & March & 5,763 & 6,877 & 492 & 165 & 197 & 14 & $-1,048$ & -491 & -30 & -14 & $-1,136$ & -579 & -32 & -17 & $-1,18$ & -623 & -34 & -18 \\
\hline & & April & 5,841 & 7,05 & 534 & 167 & 202 & 15 & 732 & 1,336 & 21 & 38 & 693 & 1,297 & 20 & 37 & -495 & 109 & -14 & 3 \\
\hline & & May & 8,065 & 9,459 & 616 & 231 & 270 & 18 & 6,572 & 7,268 & 188 & 208 & 6,507 & 7,203 & 186 & 206 & 4,555 & 5,251 & 130 & 150 \\
\hline \multirow{9}{*}{ Lima } & \multirow{5}{*}{$0-11 y$} & January & 96 & 153 & 25 & 6 & 9 & 1 & -78 & -49 & -5 & -3 & -88 & -59 & -5 & -3 & -88 & -59 & -5 & -3 \\
\hline & & February & 96 & 128 & 14 & 6 & 7 & 1 & -12 & 4 & -1 & 0 & -21 & -5 & -1 & 0 & -21 & -5 & -1 & 0 \\
\hline & & March & 128 & 160 & 14 & 8 & 9 & 1 & -77 & -61 & -5 & -4 & -81 & -65 & -5 & -4 & -81 & -65 & -5 & -4 \\
\hline & & April & 127 & 142 & 7 & 7 & 8 & 0 & -60 & -52 & -3 & -3 & -46 & -38 & -4 & -3 & -63 & -55 & -4 & -3 \\
\hline & & May & 91 & 162 & 32 & 5 & 10 & 2 & -140 & -104 & -8 & -6 & -140 & -104 & -8 & -6 & -144 & -108 & -8 & -6 \\
\hline & \multirow{4}{*}{$12-17 y$} & January & 14 & 19 & 2 & 2 & 2 & 0 & 4 & 6 & 0 & 1 & -4 & -2 & 0 & 0 & -4 & -2 & 0 & 0 \\
\hline & & February & 15 & 25 & 4 & 2 & 3 & 1 & -9 & -4 & -1 & 0 & -20 & -15 & -2 & -2 & -20 & -15 & -2 & -2 \\
\hline & & March & 10 & 22 & 5 & 1 & 3 & 1 & -15 & -9 & -2 & -1 & -17 & -11 & -2 & -1 & -17 & -11 & -2 & -1 \\
\hline & & April & 6 & 21 & 7 & 1 & 3 & 1 & -17 & -9 & -2 & -1 & -17 & -9 & -2 & -1 & -21 & -13 & -2 & -2 \\
\hline
\end{tabular}




\begin{tabular}{|c|c|c|c|c|c|c|c|c|c|c|c|c|c|c|c|c|c|c|c|c|}
\hline & & May & 15 & 24 & 4 & 2 & 3 & 0 & -16 & -11 & -2 & -1 & -17 & -12 & -2 & -1 & -18 & -13 & -2 & -2 \\
\hline & \multirow{5}{*}{$18-29 y$} & January & 83 & 92 & 4 & 4 & 5 & 0 & 12 & 16 & 1 & 1 & -41 & -37 & -2 & -2 & -41 & -37 & -2 & -2 \\
\hline & & February & 77 & 106 & 13 & 4 & 5 & 1 & -27 & -12 & -1 & -1 & -68 & -53 & -3 & -3 & -68 & -53 & -3 & -3 \\
\hline & & March & 88 & 108 & 9 & 4 & 5 & 0 & -57 & -47 & -3 & -2 & -75 & -65 & -4 & -3 & -76 & -66 & -4 & -3 \\
\hline & & April & 77 & 106 & 13 & 4 & 5 & 1 & -41 & -27 & -2 & -1 & -62 & -48 & -2 & -2 & -57 & -43 & -3 & -2 \\
\hline & & May & 65 & 109 & 19 & 3 & 5 & 1 & -19 & 3 & -1 & 0 & -23 & -1 & -1 & 0 & -30 & -8 & -1 & 0 \\
\hline & \multirow{5}{*}{$30-59 y$} & January & 423 & 570 & 65 & 11 & 15 & 2 & -106 & -33 & -3 & -1 & -190 & -117 & -5 & -3 & -190 & -117 & -5 & -3 \\
\hline & & February & 403 & 525 & 54 & 11 & 14 & 1 & -18 & 43 & 0 & 1 & -98 & -37 & -3 & -1 & -98 & -37 & -3 & -1 \\
\hline & & March & 460 & 577 & 52 & 12 & 15 & 1 & -59 & -1 & -2 & 0 & -100 & -42 & -3 & -1 & -109 & -51 & -3 & -1 \\
\hline & & April & 434 & 569 & 60 & 12 & 15 & 2 & 525 & 593 & 14 & 16 & -68 & 0 & 14 & 15 & 286 & 354 & 8 & 10 \\
\hline & & May & 422 & 575 & 67 & 11 & 15 & 2 & 1,924 & 2 & 52 & 54 & 1,91 & 1,986 & 51 & 53 & 1,63 & 1,706 & 44 & 46 \\
\hline & \multirow{5}{*}{$\geq 60 y$} & January & 423 & 570 & 65 & 121 & 176 & 25 & -390 & -54 & -32 & -5 & -432 & -96 & -36 & -8 & -432 & -96 & -36 & -8 \\
\hline & & February & 1,374 & 2,215 & 371 & 114 & 183 & 31 & -596 & -176 & -49 & -15 & -639 & -219 & -53 & -18 & -639 & -219 & -53 & -18 \\
\hline & & March & 1,935 & 2,196 & 115 & 160 & 182 & 10 & 373 & 503 & 31 & 42 & 359 & 489 & 30 & 40 & 328 & 458 & 27 & 38 \\
\hline & & April & 2,058 & 2,513 & 201 & 170 & 208 & 17 & 1,291 & 1,518 & 107 & 126 & -115 & 112 & 107 & 125 & 793 & 1,02 & 66 & 84 \\
\hline & & May & 3,115 & 3,653 & 238 & 258 & 302 & 20 & 4,328 & 4,596 & 358 & 380 & 4,322 & 4,59 & 358 & 380 & 3,6 & 3,868 & 298 & 320 \\
\hline \multirow{11}{*}{ Lambayeque } & \multirow{5}{*}{$0-11 y$} & January & -2 & 45 & 21 & -1 & 17 & 8 & -86 & -62 & -33 & -24 & -86 & -62 & -33 & -24 & -86 & -62 & -33 & -24 \\
\hline & & February & 4 & 39 & 15 & 2 & 15 & 6 & -59 & -41 & -22 & -16 & -59 & -41 & -22 & -16 & -59 & -41 & -22 & -16 \\
\hline & & March & 5 & 49 & 19 & 2 & 19 & 7 & -81 & -59 & -31 & -23 & -82 & -60 & -31 & -23 & -82 & -60 & -31 & -23 \\
\hline & & April & 10 & 30 & 9 & 4 & 11 & 3 & -46 & -36 & -18 & -14 & -46 & -36 & -18 & -14 & -46 & -36 & -18 & -14 \\
\hline & & May & 10 & 43 & 15 & 4 & 16 & 6 & -70 & -53 & -27 & -20 & -71 & -54 & -27 & -21 & -71 & -54 & -27 & -21 \\
\hline & \multirow{5}{*}{$12-17 y$} & January & 1 & 7 & 3 & 1 & 6 & 2 & -11 & -8 & -9 & -7 & -11 & -8 & -9 & -7 & -11 & -8 & -9 & -7 \\
\hline & & February & -1 & 7 & 4 & -1 & 6 & 3 & -12 & -8 & -10 & -7 & -13 & -9 & -11 & -7 & -13 & -9 & -11 & -7 \\
\hline & & March & -4 & 13 & 7 & -3 & 10 & 6 & -26 & -18 & -21 & -15 & -26 & -18 & -21 & -15 & -26 & -18 & -21 & -15 \\
\hline & & April & 1 & 6 & 2 & 1 & 5 & 2 & -9 & -7 & -7 & -6 & -9 & -7 & -7 & -6 & -9 & -7 & -7 & -6 \\
\hline & & May & 0 & 6 & 3 & 0 & 5 & 2 & -11 & -8 & -9 & -7 & -11 & -8 & -9 & -7 & -11 & -8 & -9 & -7 \\
\hline & $18-29 y$ & January & 2 & 24 & 10 & 1 & 10 & 4 & -43 & -32 & -18 & -14 & -44 & -33 & -19 & -14 & -44 & -33 & -19 & -14 \\
\hline
\end{tabular}




\begin{tabular}{|c|c|c|c|c|c|c|c|c|c|c|c|c|c|c|c|c|c|c|c|c|}
\hline & & February & 2 & 20 & 8 & 1 & 8 & 3 & -33 & -24 & -14 & -10 & -35 & -26 & -15 & -11 & -35 & -26 & -15 & -11 \\
\hline & & March & 0 & 30 & 13 & 0 & 13 & 6 & -53 & -38 & -23 & -16 & -54 & -39 & -23 & -17 & -54 & -39 & -23 & -17 \\
\hline & & April & 4 & 22 & 8 & 2 & 9 & 3 & -35 & -26 & -15 & -11 & -35 & -26 & -15 & -11 & -37 & -28 & -16 & -12 \\
\hline & & May & 5 & 27 & 10 & 2 & 12 & 4 & -41 & -30 & -18 & -13 & -42 & -31 & -18 & -13 & -42 & -31 & -18 & -13 \\
\hline & \multirow{5}{*}{$30-59 y$} & January & 8 & 128 & 53 & 2 & 30 & 12 & -217 & -157 & -51 & -37 & -222 & -162 & -52 & -38 & -222 & -162 & -52 & -38 \\
\hline & & February & 18 & 101 & 37 & 4 & 24 & 9 & -165 & -124 & -39 & -29 & -169 & -128 & -40 & -30 & -169 & -128 & -40 & -30 \\
\hline & & March & 1 & 154 & 68 & 0 & 36 & 16 & -278 & -201 & -65 & -47 & -278 & -201 & -65 & -47 & -282 & -205 & -66 & -48 \\
\hline & & April & 11 & 114 & 46 & 2 & 27 & 11 & -119 & -67 & -28 & -16 & -121 & -69 & -28 & -16 & -219 & -167 & -51 & -39 \\
\hline & & May & 15 & 122 & 47 & 4 & 28 & 11 & -27 & 27 & -6 & 6 & -29 & 25 & -7 & 6 & -105 & -51 & -25 & -12 \\
\hline & \multirow{5}{*}{$\geq 60 \mathrm{y}$} & January & -43 & 425 & 207 & -28 & 283 & 137 & -818 & -584 & -544 & -389 & -822 & -588 & -547 & -391 & -822 & -588 & -547 & -391 \\
\hline & & February & -32 & 423 & 201 & -21 & 281 & 134 & -798 & -571 & -531 & -380 & -800 & -573 & -532 & -381 & -800 & -573 & -532 & -381 \\
\hline & & March & -83 & 536 & 273 & -55 & 356 & 182 & $-1,059$ & -750 & -705 & -499 & $-1,062$ & -753 & -707 & -501 & $-1,064$ & -755 & -708 & -502 \\
\hline & & April & 20 & 407 & 171 & 14 & 271 & 114 & -569 & -375 & -378 & -250 & -571 & -377 & -380 & -251 & -794 & -600 & -528 & -400 \\
\hline & & May & 79 & 509 & 190 & 52 & 339 & 127 & -441 & -226 & -293 & -150 & -441 & -226 & -293 & -150 & -652 & -437 & -434 & -291 \\
\hline \multirow{13}{*}{ Piura } & \multirow{5}{*}{$0-11 y$} & January & 19 & 27 & 4 & 4 & 6 & 1 & -2 & 2 & -1 & 0 & -4 & 0 & -1 & 0 & -4 & 0 & -1 & 0 \\
\hline & & February & 13 & 45 & 14 & 3 & 10 & 3 & -47 & -31 & -11 & -7 & -47 & -31 & -11 & -7 & -47 & -31 & -11 & -7 \\
\hline & & March & 18 & 35 & 8 & 4 & 8 & 2 & -31 & -22 & -7 & -5 & -32 & -23 & -7 & -5 & -32 & -23 & -7 & -5 \\
\hline & & April & 26 & 34 & 4 & 6 & 8 & 1 & -26 & -22 & -6 & -5 & -26 & -22 & -6 & -5 & -28 & -24 & -6 & -5 \\
\hline & & May & 23 & 23 & 0 & 5 & 5 & 0 & -9 & -9 & -2 & -2 & -9 & -9 & -2 & -2 & -12 & -12 & -3 & -3 \\
\hline & & January & 1 & 7 & 3 & 1 & 4 & 1 & -9 & -6 & -5 & -3 & -10 & -7 & -5 & -4 & -10 & -7 & -5 & -4 \\
\hline & & February & 4 & 8 & 2 & 2 & 4 & 1 & -9 & -7 & -5 & -3 & -9 & -7 & -5 & -3 & -9 & -7 & -5 & -3 \\
\hline & $12-17 y$ & March & 2 & 5 & 1 & 1 & 2 & 1 & -4 & -3 & -2 & -1 & -4 & -3 & -2 & -1 & -4 & -3 & -2 & -1 \\
\hline & & April & 4 & 5 & 1 & 2 & 2 & 0 & -2 & -1 & -1 & -1 & -2 & -1 & -1 & -1 & -2 & -1 & -1 & -1 \\
\hline & & May & 4 & 6 & 1 & 2 & 3 & 0 & -4 & -3 & -2 & -1 & -4 & -3 & -2 & -1 & -4 & -3 & -2 & -1 \\
\hline & & January & 2 & 34 & 14 & 1 & 10 & 4 & -52 & -36 & -15 & -10 & -54 & -38 & -15 & -11 & -54 & -38 & -15 & -11 \\
\hline & $18-29 y$ & February & 8 & 26 & 8 & 2 & 8 & 2 & -29 & -19 & -8 & -6 & -32 & -22 & -9 & -6 & -32 & -22 & -9 & -6 \\
\hline & & March & 6 & 30 & 11 & 2 & 9 & 3 & -43 & -31 & -12 & -9 & -45 & -33 & -13 & -9 & -45 & -33 & -13 & -9 \\
\hline
\end{tabular}




\begin{tabular}{|c|c|c|c|c|c|c|c|c|c|c|c|c|c|c|c|c|c|c|c|c|}
\hline & & April & 14 & 16 & 1 & 4 & 5 & 0 & -7 & -6 & -2 & -2 & -8 & -7 & -2 & -2 & -9 & -8 & -3 & -2 \\
\hline & & May & 13 & 16 & 2 & 4 & 5 & 0 & 2 & 4 & 1 & 1 & -1 & 1 & 0 & 0 & -4 & -2 & -1 & -1 \\
\hline & & January & 48 & 123 & 33 & 7 & 19 & 5 & -139 & -101 & -21 & -16 & -149 & -111 & -23 & -17 & -149 & -111 & -23 & -17 \\
\hline & & February & 45 & 130 & 37 & 7 & 20 & 6 & -152 & -110 & -23 & -17 & -154 & -112 & -24 & -17 & -154 & -112 & -24 & -17 \\
\hline & $30-59 y$ & March & 59 & 131 & 32 & 9 & 20 & 5 & -145 & -109 & -22 & -17 & -153 & -117 & -23 & -18 & -153 & -117 & -23 & -18 \\
\hline & & April & 77 & 91 & 6 & 12 & 14 & 1 & 43 & 50 & 7 & 8 & 39 & 46 & 6 & 7 & -19 & -12 & -3 & -2 \\
\hline & & May & 45 & 112 & 29 & 7 & 17 & 5 & 300 & 334 & 46 & 51 & 298 & 332 & 46 & 51 & 183 & 217 & 28 & 33 \\
\hline & & January & 153 & 417 & 117 & 74 & 202 & 57 & -415 & -282 & -200 & -136 & -416 & -283 & -201 & -137 & -416 & -283 & -201 & -137 \\
\hline & & February & 140 & 415 & 121 & 68 & 200 & 59 & -458 & -321 & -221 & -155 & -462 & -325 & -223 & -157 & -462 & -325 & -223 & -157 \\
\hline & $\geq 60 y$ & March & 165 & 460 & 131 & 80 & 223 & 63 & -522 & -374 & -252 & -181 & -523 & -375 & -253 & -181 & -525 & -377 & -254 & -182 \\
\hline & & April & 280 & 397 & 52 & 135 & 192 & 25 & -114 & -56 & -55 & -27 & -116 & -58 & -56 & -28 & -233 & -175 & -113 & -84 \\
\hline & & May & 447 & 645 & 88 & 216 & 312 & 42 & 554 & 653 & 268 & 316 & 554 & 653 & 268 & 316 & 328 & 427 & 158 & 207 \\
\hline \multirow{15}{*}{ Loreto } & \multirow{5}{*}{$0-11 y$} & January & 16 & 33 & 7 & 6 & 12 & 3 & -19 & -11 & -7 & -4 & -20 & -12 & -8 & -5 & -20 & -12 & -8 & -5 \\
\hline & & February & 20 & 25 & 2 & 8 & 10 & 1 & -3 & -1 & -1 & 0 & -3 & -1 & -1 & 0 & -3 & -1 & -1 & 0 \\
\hline & & March & 14 & 32 & 8 & 5 & 12 & 3 & -30 & -20 & -11 & -8 & -31 & -21 & -12 & -8 & -31 & -21 & -12 & -8 \\
\hline & & April & 20 & 32 & 6 & 8 & 12 & 2 & -32 & -26 & -12 & -10 & -32 & -26 & -12 & -10 & -32 & -26 & -12 & -10 \\
\hline & & May & 11 & 43 & 14 & 4 & 16 & 5 & -63 & -47 & -24 & -18 & -63 & -47 & -24 & -18 & -64 & -48 & -25 & -19 \\
\hline & \multirow{5}{*}{$12-17 y$} & January & 1 & 7 & 3 & 1 & 6 & 2 & -8 & -5 & -7 & -5 & -8 & -5 & -7 & -5 & -8 & -5 & -7 & -5 \\
\hline & & February & -1 & 6 & 3 & -1 & 5 & 3 & -8 & -4 & -7 & -4 & -8 & -4 & -7 & -4 & -8 & -4 & -7 & -4 \\
\hline & & March & 2 & 3 & 1 & 2 & 3 & 1 & -1 & -1 & -1 & -1 & -1 & -1 & -1 & -1 & -1 & -1 & -1 & -1 \\
\hline & & April & 2 & 4 & 1 & 2 & 4 & 1 & -6 & -5 & -5 & -4 & -6 & -5 & -5 & -4 & -6 & -5 & -5 & -4 \\
\hline & & May & 1 & 5 & 2 & 1 & 5 & 2 & -5 & -3 & -5 & -3 & -6 & -4 & -6 & -4 & -7 & -5 & -6 & -4 \\
\hline & \multirow{5}{*}{$18-29 y$} & January & 7 & 16 & 4 & 4 & 11 & 3 & -13 & -8 & -8 & -5 & -13 & -8 & -8 & -5 & -13 & -8 & -8 & -5 \\
\hline & & February & 5 & 9 & 2 & 3 & 6 & 1 & 0 & 2 & 0 & 1 & -3 & -1 & -2 & -1 & -3 & -1 & -2 & -1 \\
\hline & & March & 7 & 13 & 3 & 4 & 8 & 2 & -12 & -9 & -7 & -6 & -12 & -9 & -7 & -6 & -12 & -9 & -7 & -6 \\
\hline & & April & 12 & 17 & 2 & 8 & 11 & 1 & -11 & -8 & -7 & -5 & -12 & -9 & -8 & -6 & -13 & -10 & -8 & -7 \\
\hline & & May & 13 & 16 & 1 & 8 & 10 & 1 & -4 & -3 & -3 & -2 & -5 & -4 & -3 & -2 & -8 & -7 & -5 & -4 \\
\hline
\end{tabular}




\begin{tabular}{|c|c|c|c|c|c|c|c|c|c|c|c|c|c|c|c|c|c|c|c|c|}
\hline & \multirow{5}{*}{$30-59 y$} & January & 39 & 49 & 4 & 14 & 17 & 1 & 12 & 17 & 4 & 6 & 8 & 13 & 3 & 5 & 8 & 13 & 3 & 5 \\
\hline & & February & 37 & 46 & 4 & 13 & 17 & 1 & -3 & 1 & -1 & 0 & -5 & -1 & -2 & 0 & -5 & -1 & -2 & 0 \\
\hline & & March & 38 & 59 & 9 & 13 & 21 & 3 & -31 & -20 & -11 & -7 & -31 & -20 & -11 & -7 & -32 & -21 & -11 & -8 \\
\hline & & April & 36 & 53 & 8 & 13 & 19 & 3 & 36 & 45 & 13 & 16 & 36 & 45 & 13 & 16 & -1 & 8 & 0 & 3 \\
\hline & & May & 45 & 69 & 11 & 16 & 25 & 4 & 174 & 186 & 62 & 67 & 173 & 185 & 62 & 66 & 121 & 133 & 43 & 48 \\
\hline & \multirow{5}{*}{$\geq 60 y$} & \begin{tabular}{|l} 
January \\
\end{tabular} & 87 & 106 & 8 & 113 & 137 & 11 & -47 & -38 & -61 & -49 & -47 & -38 & -61 & -49 & -47 & -38 & -61 & -49 \\
\hline & & February & 68 & 80 & 5 & 89 & 104 & 7 & 2 & 8 & 3 & 11 & 0 & 6 & 0 & 8 & 0 & 6 & 0 & 8 \\
\hline & & March & 75 & 97 & 10 & 97 & 125 & 12 & -6 & 5 & -7 & 7 & -7 & 4 & -8 & 6 & -8 & 3 & -10 & 4 \\
\hline & & April & 88 & 127 & 17 & 114 & 164 & 22 & 57 & 76 & 74 & 99 & 56 & 75 & 73 & 98 & -16 & 3 & -21 & 4 \\
\hline & & May & 203 & 256 & 23 & 264 & 332 & 30 & 322 & 348 & 418 & 452 & 322 & 348 & 418 & 452 & 194 & 220 & 252 & 286 \\
\hline \multirow{17}{*}{ Callao } & \multirow{5}{*}{$0-11 y$} & January & 11 & 19 & 4 & 5 & 10 & 2 & -11 & -7 & -5 & -3 & -12 & -8 & -6 & -4 & -12 & -8 & -6 & -4 \\
\hline & & February & 7 & 23 & 7 & 4 & 12 & 4 & -24 & -16 & -12 & -8 & -26 & -18 & -13 & -9 & -26 & -18 & -13 & -9 \\
\hline & & March & 17 & 20 & 2 & 5 & 11 & 3 & -14 & -12 & -12 & -9 & -16 & -14 & -13 & -10 & -16 & -14 & -13 & -10 \\
\hline & & April & 10 & 19 & 4 & 8 & 11 & 1 & -10 & -5 & -4 & -3 & -10 & -5 & -4 & -3 & -10 & -5 & -4 & -3 \\
\hline & & May & 20 & 20 & 0 & 10 & 10 & 0 & -7 & -7 & -4 & -4 & -7 & -7 & -4 & -4 & -7 & -7 & -4 & -4 \\
\hline & \multirow{5}{*}{$12-17 y$} & January & 0 & 5 & 2 & 0 & 6 & 2 & -9 & -6 & -10 & -7 & -10 & -7 & -11 & -8 & -10 & -7 & -11 & -8 \\
\hline & & February & 1 & 5 & 2 & 1 & 5 & 2 & -6 & -4 & -7 & -5 & -6 & -4 & -7 & -5 & -6 & -4 & -7 & -5 \\
\hline & & March & 0 & 1 & 1 & 0 & 2 & 1 & -1 & 0 & -3 & -2 & -1 & 0 & -3 & -2 & -1 & 0 & -3 & -2 \\
\hline & & April & 1 & 2 & 1 & 1 & 4 & 2 & -1 & -1 & -5 & -4 & -1 & -1 & -5 & -4 & -1 & -1 & -5 & -4 \\
\hline & & May & 1 & 4 & 2 & 1 & 4 & 2 & -3 & -1 & -3 & -1 & -5 & -3 & -5 & -4 & -5 & -3 & -5 & -4 \\
\hline & \multirow{5}{*}{$18-29 y$} & January & 13 & 18 & 2 & 7 & 9 & 1 & -1 & 1 & -1 & 1 & -12 & -10 & -6 & -5 & -12 & -10 & -6 & -5 \\
\hline & & February & 13 & 21 & 4 & 6 & 10 & 2 & -8 & -4 & -4 & -2 & -16 & -12 & -8 & -6 & -16 & -12 & -8 & -6 \\
\hline & & March & 8 & 13 & 2 & 4 & 7 & 1 & -3 & -1 & -3 & -2 & -4 & -2 & -4 & -2 & -5 & -3 & -4 & -3 \\
\hline & & April & 11 & 17 & 3 & 6 & 11 & 2 & -16 & -13 & -13 & -10 & -16 & -13 & -13 & -10 & -17 & -14 & -14 & -11 \\
\hline & & May & 14 & 23 & 4 & 7 & 11 & 2 & -15 & -10 & -7 & -5 & -15 & -10 & -7 & -5 & -17 & -12 & -8 & -6 \\
\hline & \multirow[t]{2}{*}{$30-59 y$} & \begin{tabular}{|l|} 
January \\
\end{tabular} & 69 & 75 & 3 & 18 & 20 & 1 & -20 & -17 & -5 & -5 & -21 & -18 & -6 & -5 & -21 & -18 & -6 & -5 \\
\hline & & February & 52 & 72 & 9 & 14 & 19 & 2 & -18 & -8 & -5 & -2 & -30 & -20 & -8 & -5 & -30 & -20 & -8 & -5 \\
\hline
\end{tabular}




\begin{tabular}{|c|c|c|c|c|c|c|c|c|c|c|c|c|c|c|c|c|c|c|c|c|}
\hline & & March & 63 & 78 & 7 & 16 & 19 & 2 & -19 & -11 & -4 & -2 & -23 & -15 & -5 & -3 & -24 & -16 & -5 & -3 \\
\hline & & April & 63 & 66 & 2 & 17 & 17 & 0 & 76 & 78 & 20 & 20 & 76 & 78 & 20 & 20 & 33 & 35 & 9 & 9 \\
\hline & & May & 65 & 83 & 8 & 17 & 22 & 2 & 258 & 267 & 68 & 70 & 256 & 265 & 67 & 69 & 193 & 202 & 51 & 53 \\
\hline & & January & 222 & 262 & 18 & 180 & 213 & 15 & -57 & -37 & -47 & -30 & -59 & -39 & -48 & -32 & -59 & -39 & -48 & -32 \\
\hline & & February & 198 & 288 & 40 & 161 & 234 & 33 & -97 & -52 & -79 & -42 & -100 & -55 & -82 & -45 & -100 & -55 & -82 & -45 \\
\hline & $\geq 60 \mathrm{y}$ & March & 241 & 287 & 20 & 192 & 228 & 16 & 150 & 159 & -26 & -8 & -39 & -16 & -26 & -8 & -41 & -18 & -27 & -9 \\
\hline & & April & 286 & 305 & 9 & 232 & 249 & 8 & 149 & 158 & 119 & 128 & 149 & 158 & 118 & 127 & 69 & 78 & 54 & 62 \\
\hline & & May & 503 & 525 & 10 & 408 & 427 & 8 & 742 & 754 & 603 & 612 & 740 & 752 & 601 & 610 & 604 & 616 & 491 & 500 \\
\hline \multirow{19}{*}{ Ancash } & \multirow{5}{*}{$0-11 y$} & January & 13 & 27 & 6 & 6 & 11 & 3 & -16 & -9 & -7 & -4 & -20 & -13 & -8 & -6 & -20 & -13 & -8 & -6 \\
\hline & & February & 18 & 28 & 4 & 8 & 12 & 2 & -18 & -13 & -8 & -5 & -20 & -15 & -8 & -6 & -20 & -15 & -8 & -6 \\
\hline & & March & 16 & 31 & 7 & 7 & 13 & 3 & -27 & -19 & -11 & -8 & -30 & -22 & -13 & -9 & -30 & -22 & -13 & -9 \\
\hline & & April & 13 & 27 & 6 & 6 & 11 & 3 & -20 & -13 & -8 & -6 & -22 & -15 & -9 & -6 & -22 & -15 & -9 & -6 \\
\hline & & May & 19 & 27 & 4 & 8 & 12 & 2 & -13 & -9 & -6 & -4 & -13 & -9 & -6 & -4 & -13 & -9 & -6 & -4 \\
\hline & \multirow{5}{*}{$12-17 y$} & January & 2 & 3 & 1 & 2 & 3 & 1 & -1 & -1 & -1 & -1 & -2 & -2 & -2 & -2 & -2 & -2 & -2 & -2 \\
\hline & & February & 3 & 4 & 1 & 3 & 4 & 1 & -3 & -3 & -3 & -2 & -3 & -3 & -3 & -2 & -3 & -3 & -3 & -2 \\
\hline & & March & 4 & 6 & 1 & 3 & 5 & 1 & -3 & -2 & -3 & -2 & -4 & -3 & -4 & -3 & -4 & -3 & -4 & -3 \\
\hline & & April & 3 & 6 & 2 & 2 & 5 & 1 & -4 & -2 & -4 & -2 & -5 & -3 & -4 & -3 & -5 & -3 & -4 & -3 \\
\hline & & May & 3 & 6 & 2 & 3 & 6 & 1 & -6 & -5 & -6 & -4 & -6 & -5 & -6 & -4 & -6 & -5 & -6 & -4 \\
\hline & \multirow{5}{*}{$18-29 y$} & January & 15 & 20 & 2 & 8 & 10 & 1 & -8 & -5 & -4 & -3 & -15 & -12 & -8 & -6 & -15 & -12 & -8 & -6 \\
\hline & & February & 9 & 20 & 5 & 5 & 10 & 3 & -16 & -11 & -8 & -5 & -21 & -16 & -11 & -8 & -21 & -16 & -11 & -8 \\
\hline & & March & 11 & 17 & 3 & 6 & 9 & 1 & -11 & -8 & -6 & -4 & -15 & -12 & -8 & -6 & -15 & -12 & -8 & -6 \\
\hline & & April & 12 & 17 & 2 & 6 & 9 & 1 & -11 & -8 & -6 & -4 & -13 & -10 & -7 & -5 & -13 & -10 & -7 & -5 \\
\hline & & May & 7 & 20 & 6 & 3 & 10 & 3 & -16 & -9 & -8 & -5 & -19 & -12 & -9 & -6 & -19 & -12 & -9 & -6 \\
\hline & \multirow{4}{*}{$30-59 y$} & January & 53 & 94 & 18 & 13 & 24 & 5 & -42 & -22 & -11 & -6 & -55 & -35 & -14 & -9 & -55 & -35 & -14 & -9 \\
\hline & & February & 49 & 73 & 11 & 12 & 19 & 3 & -24 & -12 & -6 & -3 & -36 & -24 & -9 & -6 & -36 & -24 & -9 & -6 \\
\hline & & March & 52 & 80 & 13 & 13 & 20 & 3 & -43 & -29 & -11 & -7 & -47 & -33 & -12 & -8 & -47 & -33 & -12 & -8 \\
\hline & & April & 51 & 76 & 11 & 13 & 19 & 3 & -16 & -4 & -4 & -1 & -18 & -6 & -5 & -1 & -41 & -29 & -11 & -7 \\
\hline
\end{tabular}




\begin{tabular}{|c|c|c|c|c|c|c|c|c|c|c|c|c|c|c|c|c|c|c|c|c|}
\hline & & May & 75 & 95 & 9 & 19 & 24 & 2 & 84 & 94 & 22 & 24 & 81 & 91 & 21 & 23 & 29 & 39 & 7 & 10 \\
\hline & & January & 204 & 429 & 100 & 134 & 287 & 68 & -291 & -178 & -197 & -121 & -296 & -183 & -201 & -124 & -296 & -183 & -201 & -124 \\
\hline & & February & 198 & 335 & 61 & 134 & 228 & 41 & -211 & -142 & -143 & -96 & -216 & -147 & -146 & -100 & -216 & -147 & -146 & -100 \\
\hline & $\geq 60 y$ & March & 232 & 399 & 74 & 158 & 271 & 50 & -179 & -96 & -122 & -65 & -186 & -103 & -126 & -70 & -187 & -104 & -127 & -70 \\
\hline & & April & 209 & 387 & 79 & 142 & 263 & 53 & -156 & -67 & -106 & -45 & -157 & -68 & -106 & -46 & -215 & -126 & -146 & -85 \\
\hline & & May & 377 & 453 & 34 & 256 & 308 & 23 & 202 & 241 & 137 & 163 & 197 & 236 & 134 & 160 & 61 & 100 & 42 & 68 \\
\hline \multirow{21}{*}{ Ica } & \multirow{5}{*}{$0-11 y$} & January & 11 & 20 & 4 & 6 & 11 & 2 & -5 & 0 & -2 & 0 & -7 & -2 & -3 & -1 & -7 & -2 & -3 & -1 \\
\hline & & February & 10 & 27 & 7 & 5 & 14 & 4 & -25 & -17 & -13 & -9 & -26 & -18 & -14 & -10 & -26 & -18 & -14 & -10 \\
\hline & & March & 17 & 26 & 4 & 9 & 14 & 2 & -26 & -22 & -14 & -12 & -27 & -23 & -15 & -12 & -27 & -23 & -15 & -12 \\
\hline & & April & 13 & 16 & 2 & 7 & 9 & 1 & -7 & -6 & -4 & -3 & -7 & -6 & -4 & -3 & -8 & -7 & -4 & -4 \\
\hline & & May & 10 & 20 & 5 & 5 & 10 & 2 & -13 & -8 & -7 & -4 & -13 & -8 & -7 & -4 & -13 & -8 & -7 & -4 \\
\hline & \multirow{5}{*}{$12-17 y$} & January & 1 & 2 & 1 & 1 & 2 & 1 & 3 & 4 & 3 & 4 & 1 & 2 & 1 & 2 & 1 & 2 & 1 & 2 \\
\hline & & February & 0 & 5 & 2 & 0 & 6 & 3 & -7 & -4 & -8 & -5 & -7 & -4 & -8 & -5 & -7 & -4 & -8 & -5 \\
\hline & & March & 1 & 5 & 2 & 1 & 6 & 2 & -8 & -6 & -10 & -8 & -8 & -6 & -10 & -8 & -8 & -6 & -10 & -8 \\
\hline & & April & 2 & 3 & 1 & 2 & 4 & 1 & 1 & 1 & 1 & 1 & -1 & -1 & -2 & -1 & -1 & -1 & -2 & -1 \\
\hline & & May & 1 & 3 & 1 & 1 & 4 & 1 & -4 & -3 & -5 & -4 & -4 & -3 & -5 & -4 & -4 & -3 & -5 & -4 \\
\hline & \multirow{5}{*}{$18-29 y$} & January & 8 & 14 & 3 & 5 & 8 & 2 & 0 & 3 & 0 & 2 & -10 & -7 & -6 & -4 & -10 & -7 & -6 & -4 \\
\hline & & February & 11 & 14 & 2 & 6 & 8 & 1 & 3 & 4 & 1 & 2 & -6 & -5 & -4 & -3 & -6 & -5 & -4 & -3 \\
\hline & & March & 12 & 17 & 2 & 7 & 10 & 1 & -14 & -12 & -8 & -7 & -19 & -17 & -11 & -10 & -19 & -17 & -11 & -10 \\
\hline & & April & 5 & 19 & 6 & 3 & 11 & 4 & -23 & -15 & -13 & -9 & -24 & -16 & -14 & -10 & -25 & -17 & -14 & -10 \\
\hline & & May & 11 & 16 & 2 & 6 & 9 & 1 & -9 & -6 & -5 & -3 & -14 & -11 & -8 & -6 & -14 & -11 & -8 & -6 \\
\hline & \multirow{5}{*}{$30-59 y$} & January & 42 & 74 & 15 & 13 & 24 & 5 & -4 & 12 & -1 & 4 & -27 & -11 & -9 & -4 & -27 & -11 & -9 & -4 \\
\hline & & February & 43 & 66 & 10 & 14 & 21 & 3 & -8 & 4 & -2 & 1 & -24 & -12 & -8 & -4 & -24 & -12 & -8 & -4 \\
\hline & & March & 57 & 77 & 9 & 18 & 25 & 3 & -38 & -27 & -12 & -9 & -44 & -33 & -14 & -11 & -44 & -33 & -14 & -11 \\
\hline & & April & 42 & 75 & 15 & 14 & 24 & 5 & -19 & -2 & -6 & -1 & -22 & -5 & -7 & -2 & -45 & -28 & -15 & -9 \\
\hline & & May & 54 & 76 & 10 & 18 & 25 & 3 & 102 & 113 & 33 & 36 & 96 & 107 & 31 & 35 & 30 & 41 & 10 & 13 \\
\hline & $\geq 60 y$ & January & 219 & 267 & 22 & 221 & 270 & 22 & -89 & -65 & -90 & -66 & -89 & -65 & -90 & -66 & -89 & -65 & -90 & -66 \\
\hline
\end{tabular}




\begin{tabular}{|c|c|c|c|c|c|c|c|c|c|c|c|c|c|c|c|c|c|c|c|c|}
\hline & & February & 202 & 310 & 48 & 204 & 314 & 49 & -139 & -84 & -140 & -85 & -144 & -89 & -145 & -90 & -144 & -89 & -145 & -90 \\
\hline & & March & 229 & 306 & 34 & 232 & 310 & 34 & -107 & -69 & -108 & -69 & -110 & -72 & -111 & -72 & -110 & -72 & -111 & -72 \\
\hline & & April & 219 & 285 & 29 & 221 & 288 & 29 & -56 & -23 & -57 & -23 & -58 & -25 & -59 & -25 & -96 & -63 & -97 & -64 \\
\hline & & May & 321 & 364 & 19 & 324 & 368 & 19 & 179 & 200 & 180 & 202 & 177 & 198 & 178 & 200 & 54 & 75 & 54 & 76 \\
\hline \multirow{23}{*}{ La Libertad } & \multirow{5}{*}{$0-11 y$} & January & 37 & 40 & 1 & 9 & 10 & 0 & -1 & 0 & 0 & 0 & -3 & -2 & -1 & 0 & -3 & -2 & -1 & 0 \\
\hline & & February & 27 & 43 & 7 & 7 & 11 & 2 & -29 & -21 & -7 & -5 & -33 & -25 & -8 & -6 & -33 & -25 & -8 & -6 \\
\hline & & March & 34 & 47 & 6 & 9 & 12 & 1 & -33 & -27 & -8 & -7 & -34 & -28 & -9 & -7 & -34 & -28 & -9 & -7 \\
\hline & & April & 28 & 35 & 3 & 7 & 9 & 1 & -15 & -12 & -4 & -3 & -16 & -13 & -4 & -3 & -16 & -13 & -4 & -3 \\
\hline & & May & 27 & 51 & 11 & 7 & 13 & 3 & -49 & -37 & -12 & -9 & -49 & -37 & -12 & -9 & -50 & -38 & -13 & -10 \\
\hline & \multirow{5}{*}{$12-17 y$} & January & 7 & 10 & 2 & 4 & 5 & 1 & -4 & -2 & -2 & -1 & -5 & -3 & -3 & -2 & -5 & -3 & -3 & -2 \\
\hline & & February & 5 & 7 & 1 & 3 & 4 & 1 & -1 & 0 & -1 & 0 & -5 & -4 & -3 & -2 & -5 & -4 & -3 & -2 \\
\hline & & March & 6 & 13 & 3 & 3 & 7 & 2 & -14 & -11 & -8 & -6 & -17 & -14 & -9 & -7 & -17 & -14 & -9 & -7 \\
\hline & & April & 3 & 9 & 3 & 2 & 5 & 1 & -10 & -7 & -6 & -4 & -10 & -7 & -6 & -4 & -11 & -8 & -6 & -5 \\
\hline & & May & 3 & 9 & 3 & 2 & 5 & 1 & -9 & -6 & -5 & -3 & -11 & -8 & -6 & -5 & -11 & -8 & -6 & -5 \\
\hline & \multirow{5}{*}{$18-29 y$} & January & 22 & 37 & 7 & 6 & 10 & 2 & -15 & -8 & -4 & -2 & -33 & -26 & -9 & -7 & -33 & -26 & -9 & -7 \\
\hline & & February & 22 & 40 & 8 & 6 & 11 & 2 & -36 & -26 & -10 & -7 & -42 & -32 & -12 & -9 & -42 & -32 & -12 & -9 \\
\hline & & March & 25 & 35 & 5 & 7 & 10 & 1 & -26 & -21 & -7 & -6 & -32 & -27 & -9 & -8 & -32 & -27 & -9 & -8 \\
\hline & & April & 16 & 27 & 5 & 4 & 8 & 1 & -25 & -19 & -7 & -5 & -27 & -21 & -8 & -6 & -27 & -21 & -8 & -6 \\
\hline & & May & 23 & 31 & 4 & 6 & 9 & 1 & -11 & -7 & -3 & -2 & -19 & -15 & -5 & -4 & -19 & -15 & -5 & -4 \\
\hline & \multirow{5}{*}{$30-59 y$} & January & 108 & 135 & 12 & 17 & 22 & 2 & -23 & -10 & -4 & -2 & -38 & -25 & -6 & -4 & -38 & -25 & -6 & -4 \\
\hline & & February & 103 & 128 & 11 & 16 & 21 & 2 & -56 & -43 & -9 & -7 & -73 & -60 & -12 & -10 & -73 & -60 & -12 & -10 \\
\hline & & March & 95 & 154 & 26 & 15 & 25 & 4 & -113 & -84 & -18 & -13 & -129 & -100 & -21 & -16 & -131 & -102 & -21 & -16 \\
\hline & & April & 109 & 129 & 9 & 17 & 21 & 1 & -38 & -28 & -6 & -5 & -42 & -32 & -7 & -5 & -55 & -45 & -9 & -7 \\
\hline & & May & 108 & 125 & 7 & 17 & 20 & 1 & 74 & 82 & 12 & 13 & 67 & 75 & 11 & 12 & 25 & 33 & 4 & 5 \\
\hline & \multirow{3}{*}{$\geq 60 y$} & January & 398 & 523 & 55 & 187 & 245 & 26 & -146 & -84 & -68 & -39 & -153 & -91 & -72 & -43 & -153 & -91 & -72 & -43 \\
\hline & & February & 336 & 564 & 101 & 158 & 265 & 47 & -362 & -248 & -170 & -116 & -366 & -252 & -172 & -118 & -366 & -252 & -172 & -118 \\
\hline & & March & 376 & 578 & 89 & 176 & 271 & 42 & -314 & -213 & -147 & -100 & -315 & -214 & -148 & -100 & -316 & -215 & -148 & -101 \\
\hline
\end{tabular}




\begin{tabular}{|c|c|c|c|c|c|c|c|c|c|c|c|c|c|c|c|c|c|c|c|c|}
\hline & & April & 378 & 454 & 33 & 177 & 213 & 16 & -132 & -94 & -62 & -44 & -133 & -95 & -62 & -44 & -166 & -128 & -78 & -60 \\
\hline & & May & 497 & 509 & 5 & 233 & 238 & 2 & 144 & 150 & 68 & 70 & 141 & 147 & 66 & 69 & 35 & 41 & 17 & 19 \\
\hline \multirow{25}{*}{ Ucayali } & \multirow{5}{*}{$0-11 y$} & January & 13 & 13 & 0 & 9 & 9 & 0 & 1 & 1 & 1 & 1 & 1 & 1 & 1 & 1 & 1 & 1 & 1 & 1 \\
\hline & & February & 4 & 13 & 4 & 3 & 9 & 3 & -10 & -6 & -7 & -4 & -10 & -6 & -7 & -4 & -10 & -6 & -7 & -4 \\
\hline & & March & 2 & 15 & 6 & 1 & 11 & 4 & -11 & -4 & -8 & -3 & -11 & -4 & -8 & -3 & -11 & -4 & -8 & -3 \\
\hline & & April & 8 & 10 & 1 & 6 & 7 & 1 & 1 & 2 & 1 & 1 & 1 & 2 & 1 & 1 & 1 & 2 & 1 & 1 \\
\hline & & May & 6 & 24 & 8 & 4 & 17 & 6 & -29 & -20 & -21 & -14 & -29 & -20 & -21 & -14 & -31 & -22 & -22 & -16 \\
\hline & \multirow{5}{*}{$12-17 y$} & January & 1 & 3 & 1 & 2 & 6 & 2 & -3 & -2 & -6 & -4 & -3 & -2 & -6 & -4 & -3 & -2 & -6 & -4 \\
\hline & & February & 1 & 2 & 1 & 2 & 4 & 1 & -3 & -3 & -6 & -5 & -3 & -3 & -6 & -5 & -3 & -3 & -6 & -5 \\
\hline & & March & 0 & 3 & 1 & 0 & 5 & 2 & -1 & 0 & -2 & 1 & -1 & 0 & -2 & 1 & -1 & 0 & -2 & 1 \\
\hline & & April & 0 & 1 & 1 & -1 & 2 & 1 & 2 & 3 & 3 & 4 & 2 & 3 & 3 & 4 & 2 & 3 & 3 & 4 \\
\hline & & May & 0 & 6 & 3 & 0 & 10 & 4 & -11 & -8 & -19 & -14 & -11 & -8 & -19 & -14 & -11 & -8 & -19 & -14 \\
\hline & \multirow{5}{*}{$18-29 y$} & \begin{tabular}{|l} 
January \\
\end{tabular} & 3 & 18 & 7 & 3 & 19 & 7 & -25 & -18 & -26 & -18 & -25 & -18 & -26 & -18 & -25 & -18 & -26 & -18 \\
\hline & & February & 7 & 14 & 3 & 7 & 15 & 3 & -8 & -5 & -9 & -5 & -8 & -5 & -9 & -5 & -8 & -5 & -9 & -5 \\
\hline & & March & 3 & 10 & 3 & 3 & 10 & 3 & -3 & 1 & -3 & 1 & -5 & -1 & -5 & -1 & -5 & -1 & -5 & -1 \\
\hline & & April & 6 & 6 & 0 & 6 & 6 & 0 & 12 & 12 & 12 & 12 & 12 & 12 & 12 & 12 & 10 & 10 & 10 & 10 \\
\hline & & May & 7 & 17 & 5 & 7 & 18 & 5 & -17 & -12 & -18 & -13 & -17 & -12 & -18 & -13 & -18 & -13 & -19 & -14 \\
\hline & \multirow{5}{*}{$30-59 y$} & January & 37 & 51 & 6 & 22 & 31 & 4 & -2 & 6 & -1 & 3 & -3 & 5 & -2 & 3 & -3 & 5 & -2 & 3 \\
\hline & & February & 23 & 55 & 14 & 14 & 33 & 9 & -47 & -31 & -29 & -19 & -50 & -34 & -30 & -21 & -50 & -34 & -30 & -21 \\
\hline & & March & 13 & 37 & 11 & 8 & 23 & 6 & -15 & -3 & -9 & -2 & -16 & -4 & -9 & -2 & -16 & -4 & -9 & -2 \\
\hline & & April & 22 & 40 & 8 & 14 & 25 & 5 & 37 & 46 & 22 & 28 & 36 & 45 & 22 & 27 & 20 & 29 & 12 & 17 \\
\hline & & May & 13 & 48 & 16 & 8 & 29 & 10 & 92 & 110 & 56 & 67 & 92 & 110 & 56 & 67 & 75 & 93 & 46 & 56 \\
\hline & \multirow{5}{*}{$\geq 60 \mathrm{y}$} & January & 46 & 75 & 13 & 118 & 194 & 34 & -91 & -76 & -233 & -195 & -91 & -76 & -233 & -195 & -91 & -76 & -233 & -195 \\
\hline & & February & 50 & 95 & 20 & 127 & 246 & 52 & -51 & -28 & -131 & -72 & -51 & -28 & -131 & -72 & -51 & -28 & -131 & -72 \\
\hline & & March & 44 & 99 & 24 & 113 & 255 & 63 & -55 & -27 & -141 & -70 & -55 & -27 & -141 & -70 & -55 & -27 & -141 & -70 \\
\hline & & April & 82 & 138 & 25 & 212 & 356 & 64 & 44 & 72 & 114 & 186 & 44 & 72 & 114 & 186 & 13 & 41 & 34 & 106 \\
\hline & & May & 156 & 219 & 28 & 402 & 565 & 72 & 240 & 272 & 619 & 700 & 240 & 272 & 619 & 700 & 203 & 235 & 524 & 605 \\
\hline
\end{tabular}


80 TD: Total deaths, VD: Violent deaths, CV: COVID-19 deaths

$$
\begin{aligned}
& 81 \\
& 82 \\
& 83 \\
& 84 \\
& 85 \\
& 86 \\
& 87 \\
& 88 \\
& 89 \\
& 90 \\
& 91 \\
& 92 \\
& 93
\end{aligned}
$$




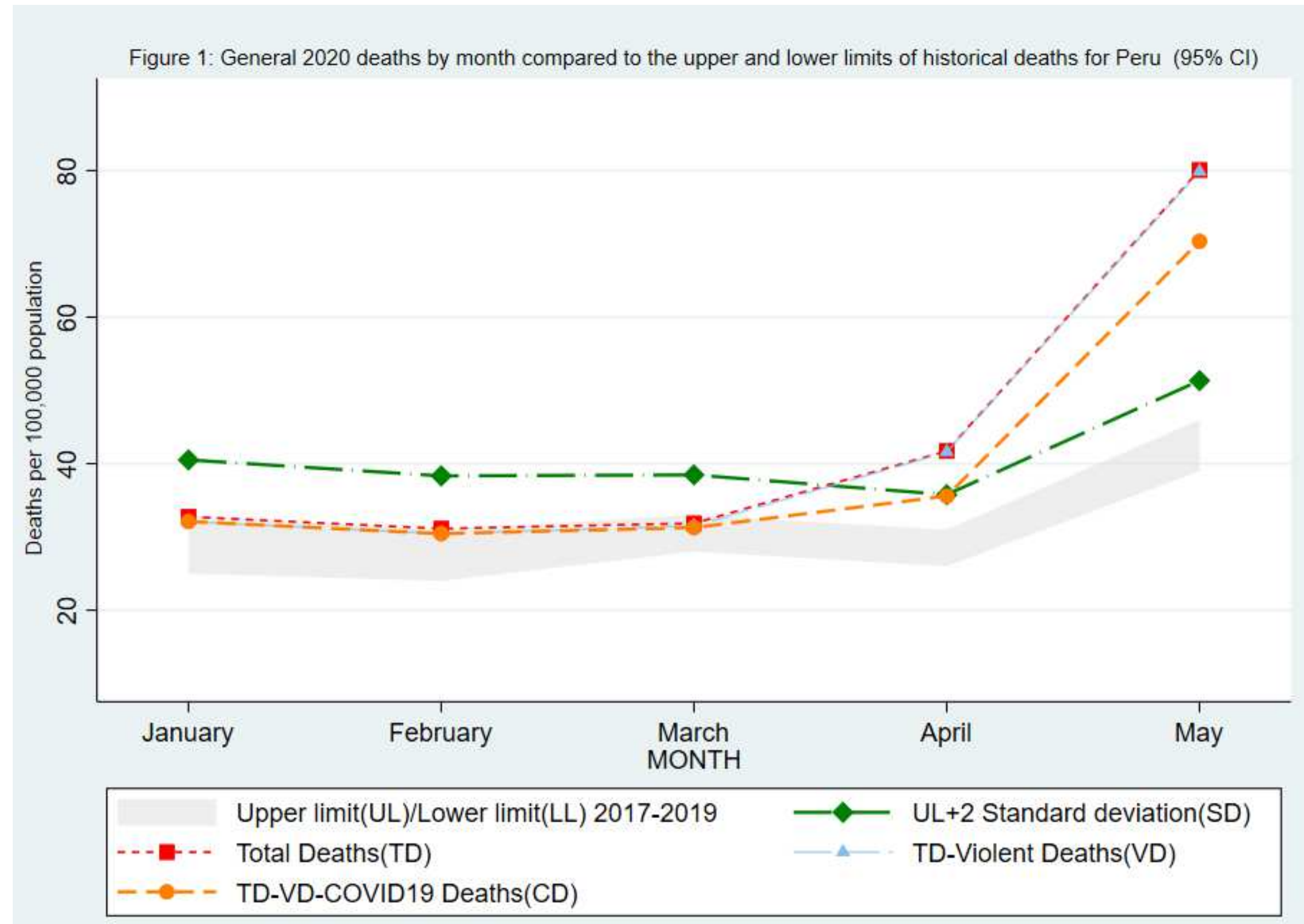




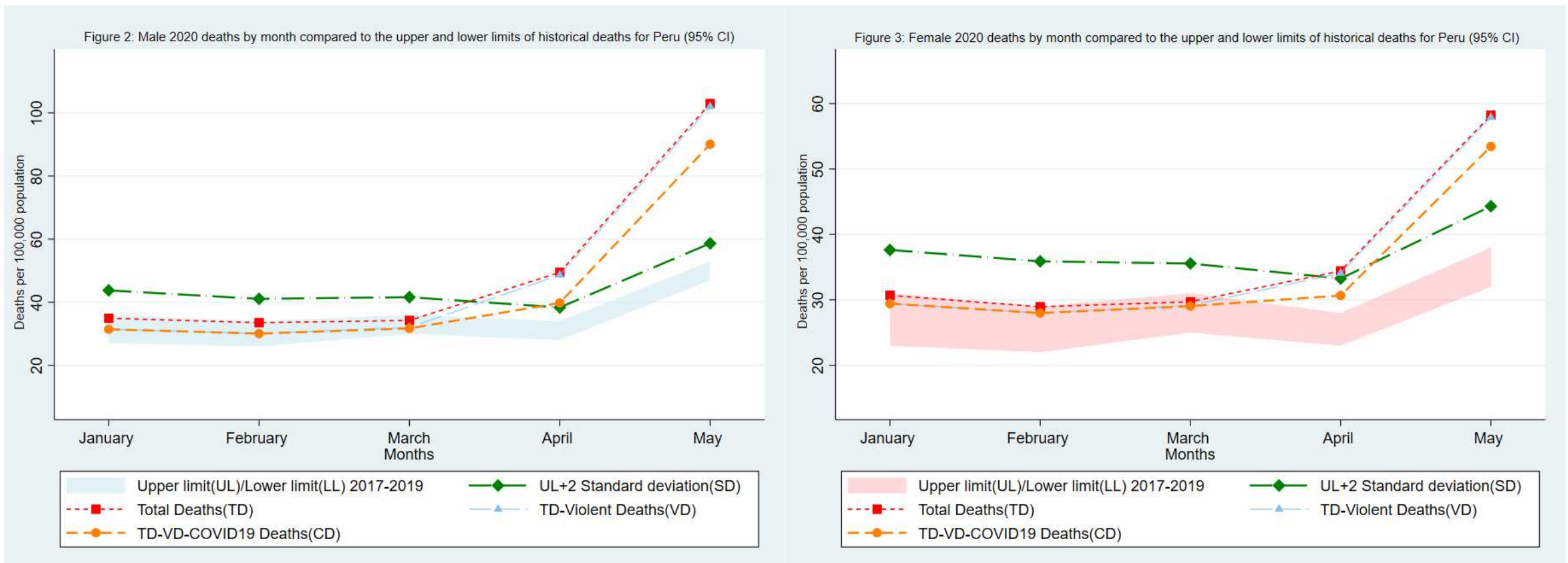



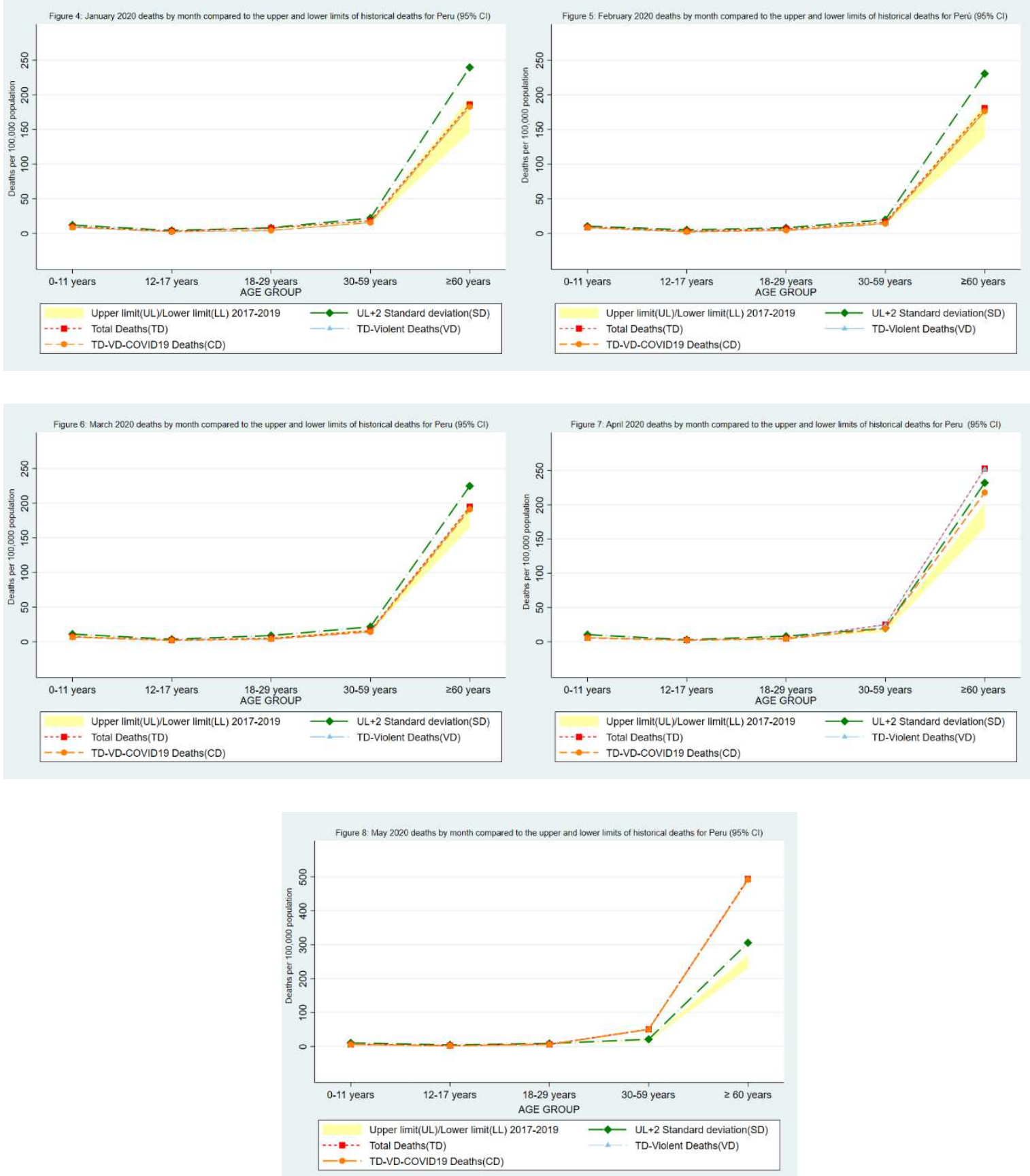


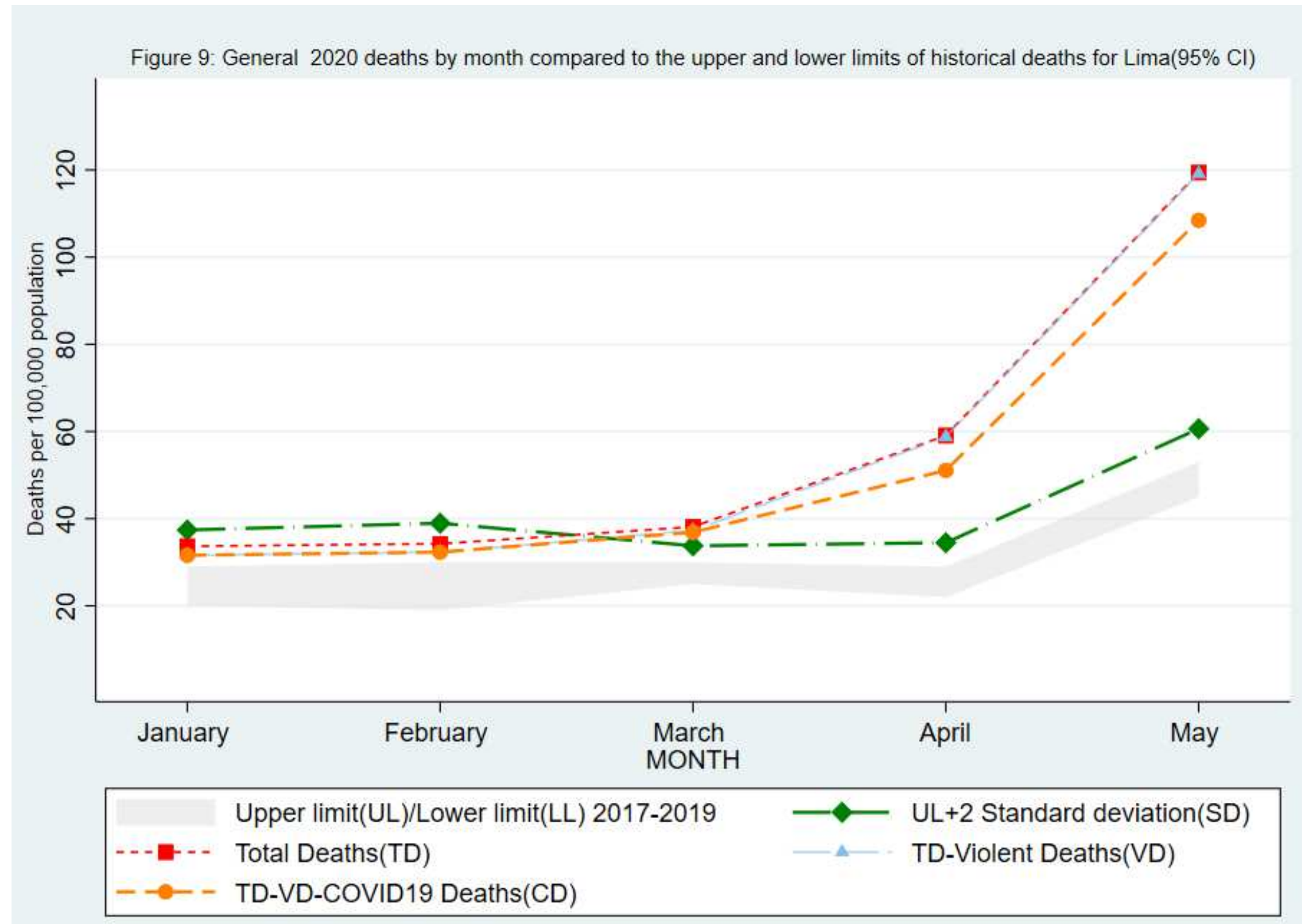



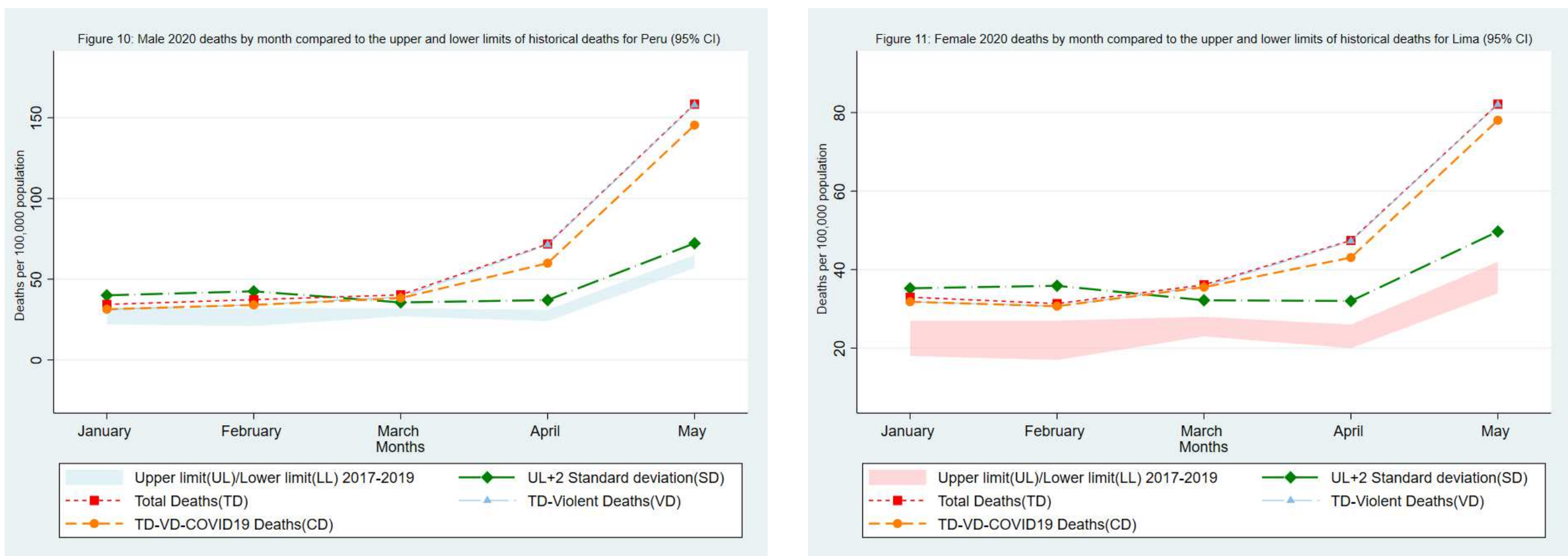

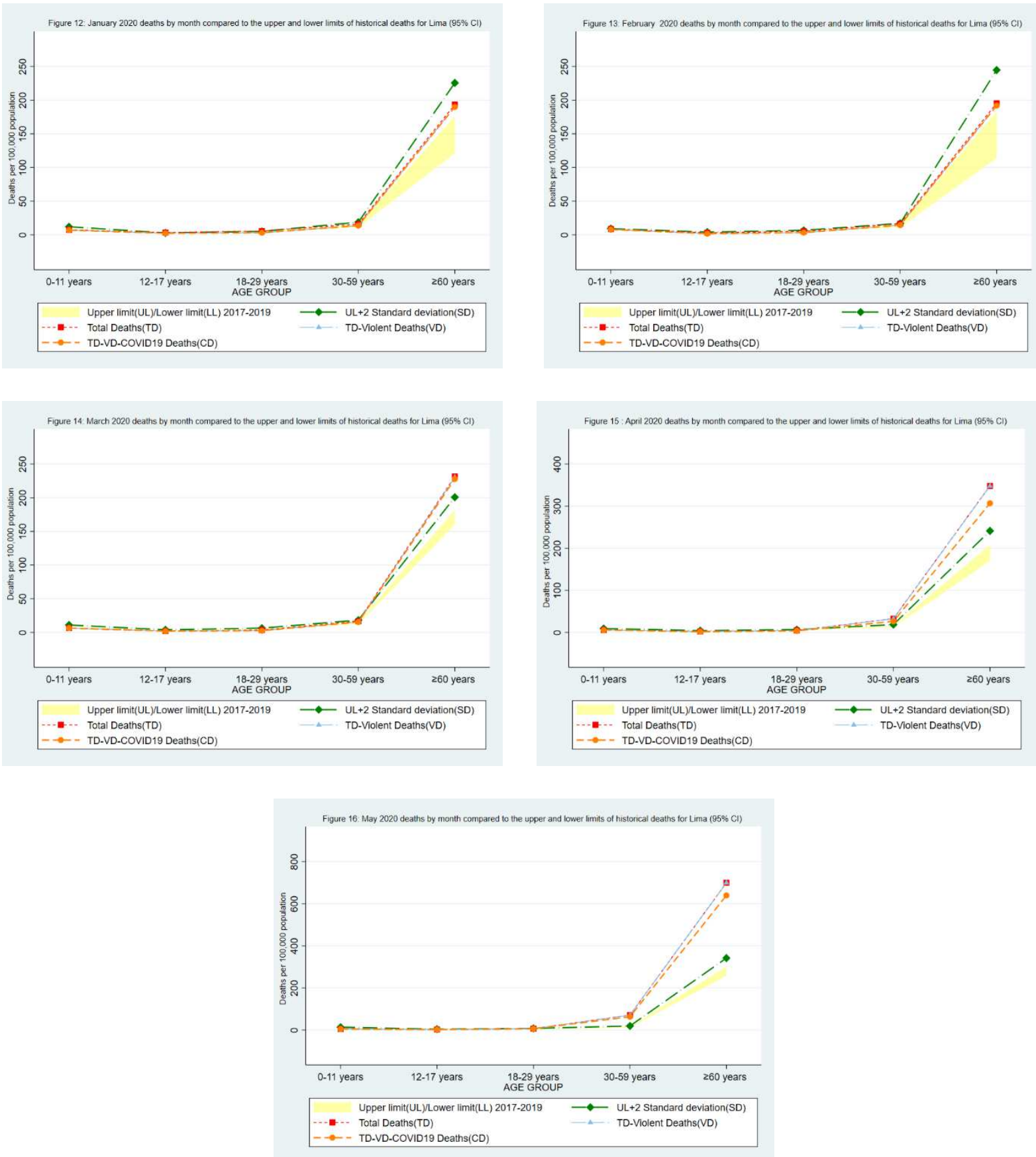
Figure 17: General 2020 deaths by month compared to the upper and lower limits of historical deaths for Lambayeque( $95 \% \mathrm{Cl})$

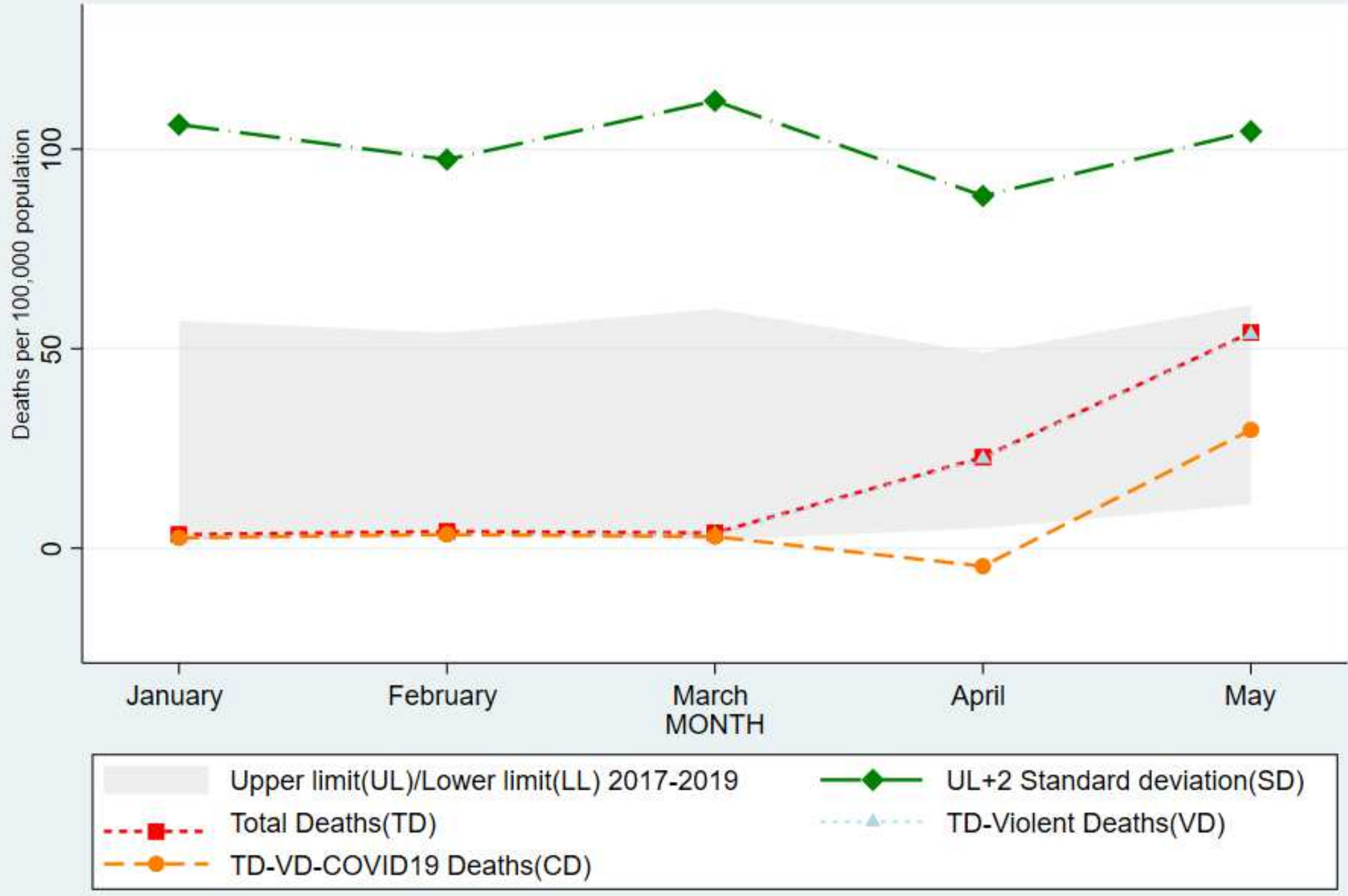


Figure 18: Male 2020 deaths by month compared to the upper and lower limits of historical deaths for Lambayeque $(95 \% \mathrm{Cl})$

$\stackrel{\circ}{\leftarrow}$

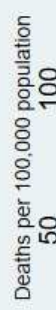

$\circ$
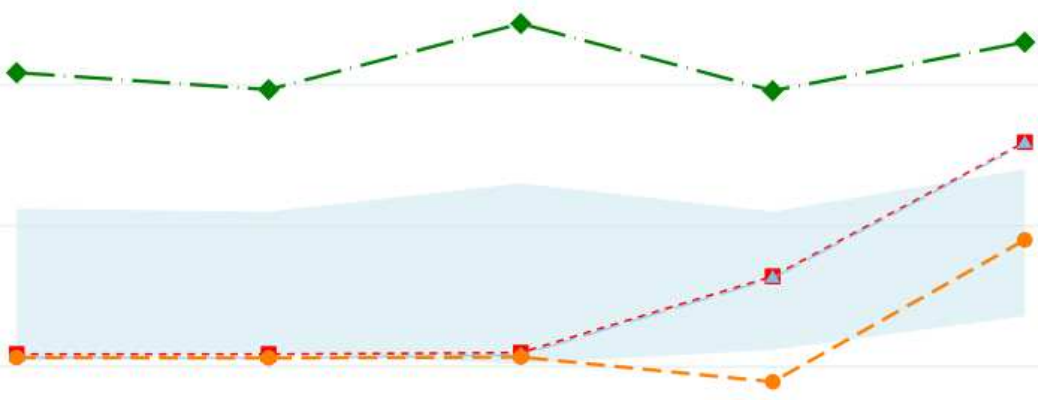

January

February

March
Months

April

May

Upper limit(UL)/Lower limit(LL) 2017-2019

$\longrightarrow$ UL+2 Standard deviation(SD)

-. - Total Deaths(TD)

TD-Violent Deaths(VD)

$-\rightarrow-$ TD-VD-COVID19 Deaths(CD)
Figure 19: Female 2020 deaths by month compared to the upper and lower limits of historical deaths for Lima $(95 \% \mathrm{Cl})$

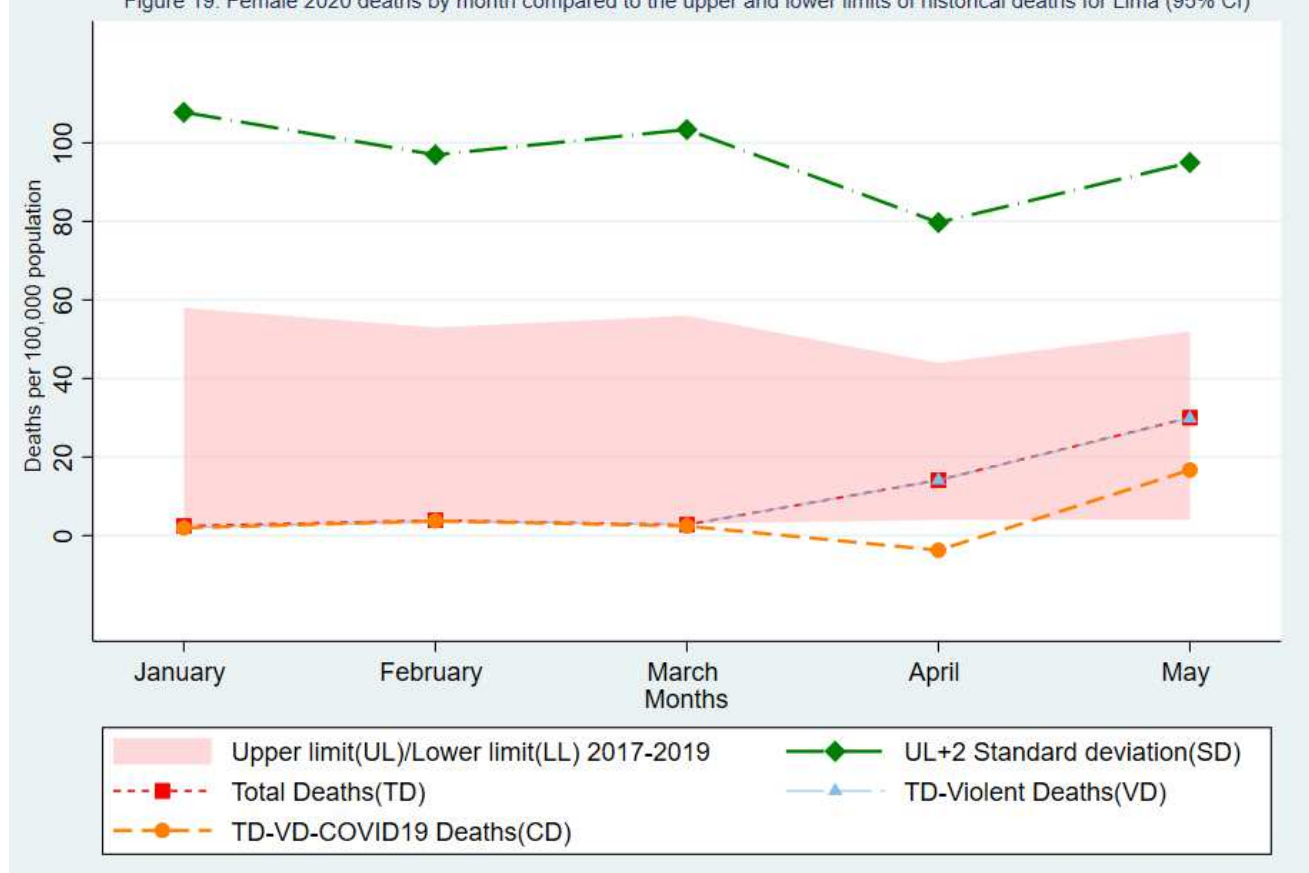



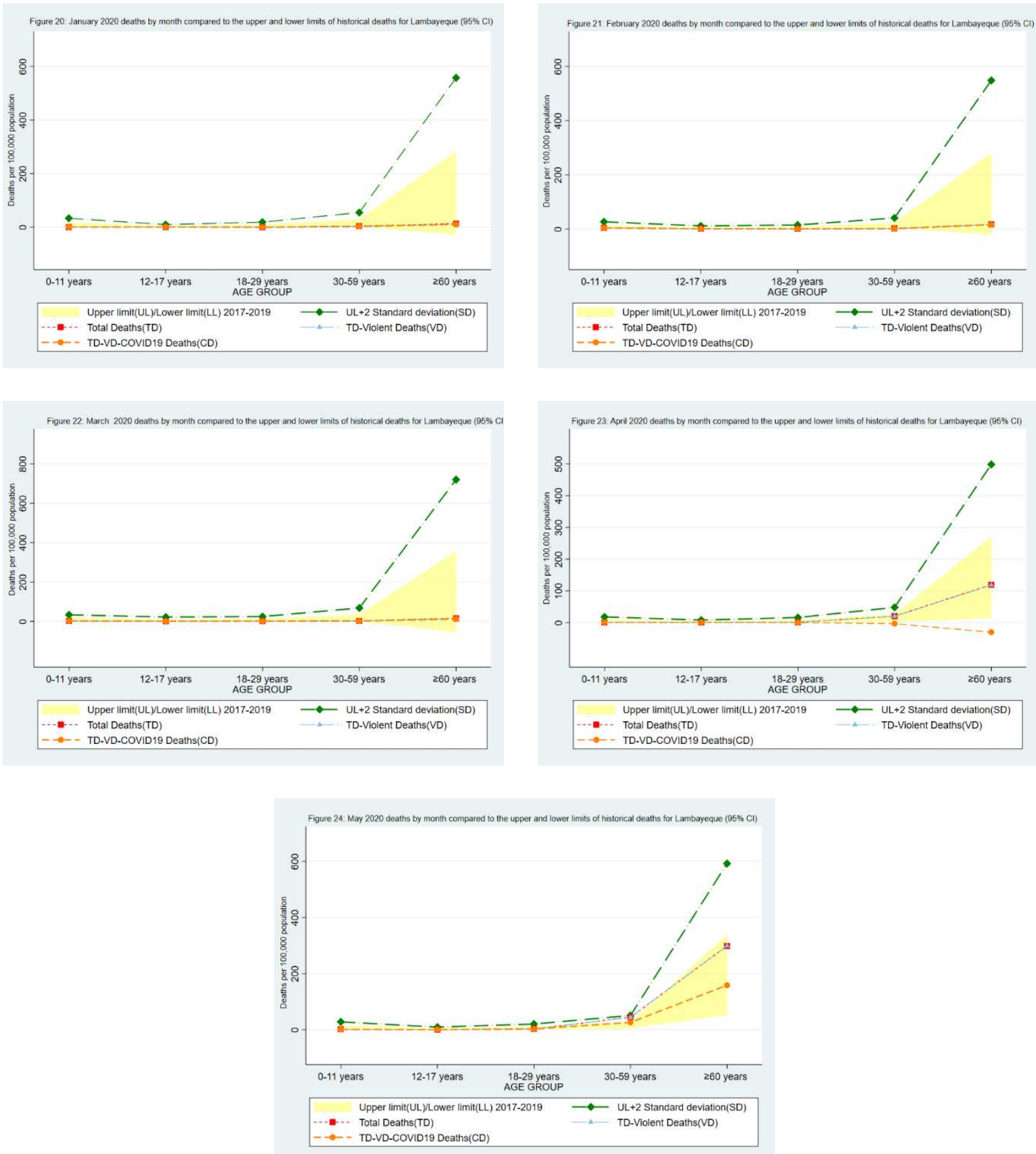


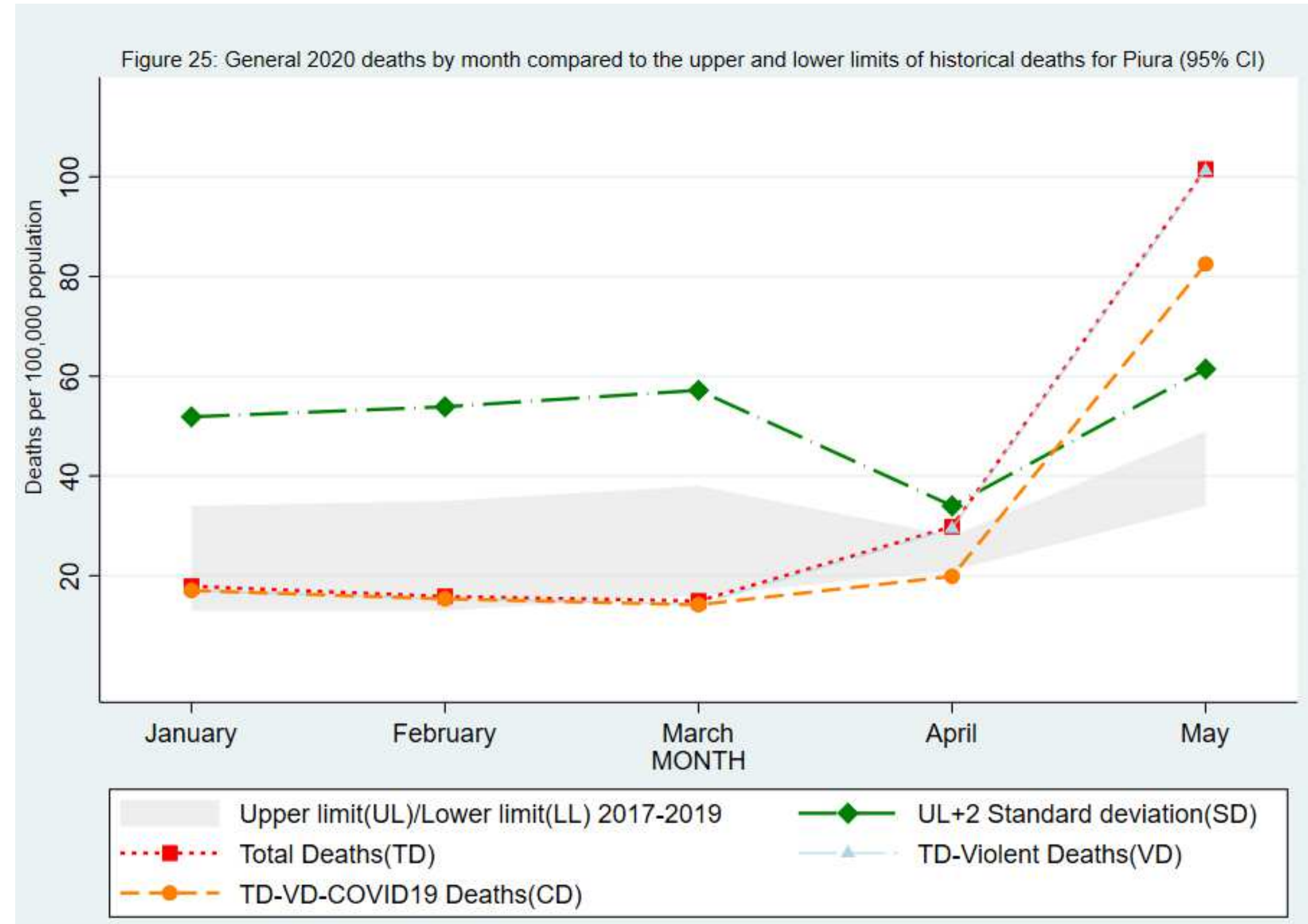



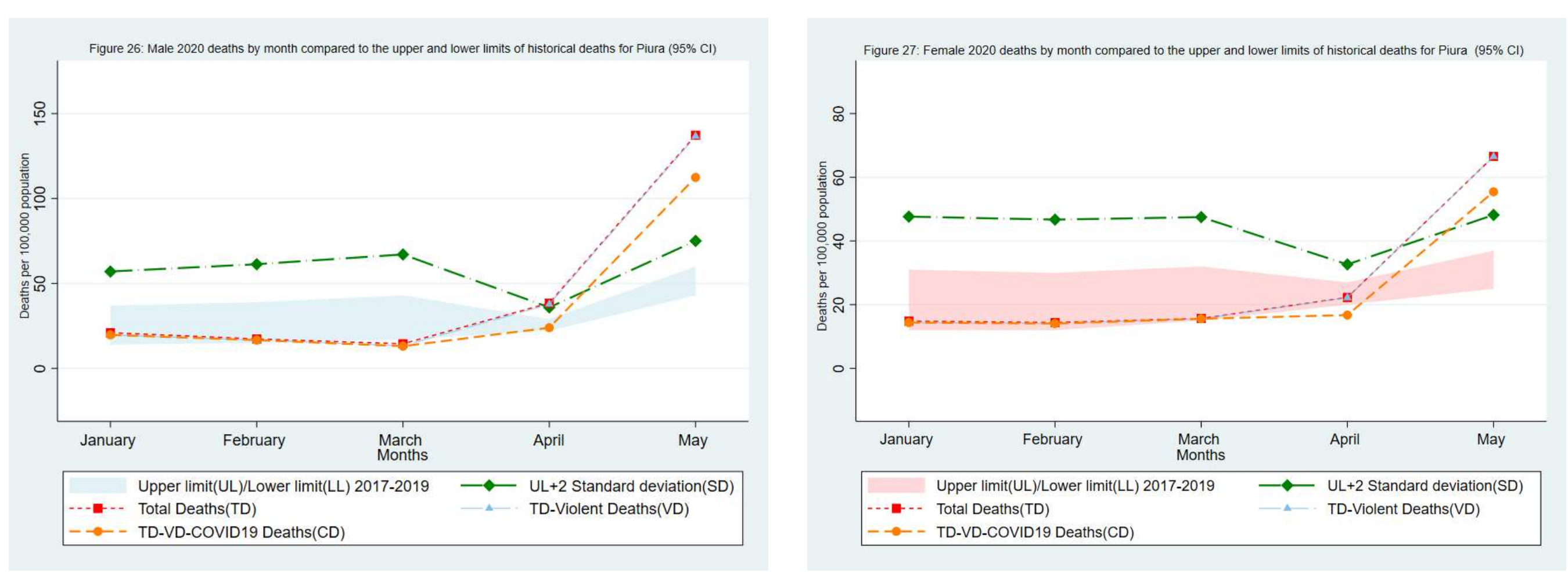
524
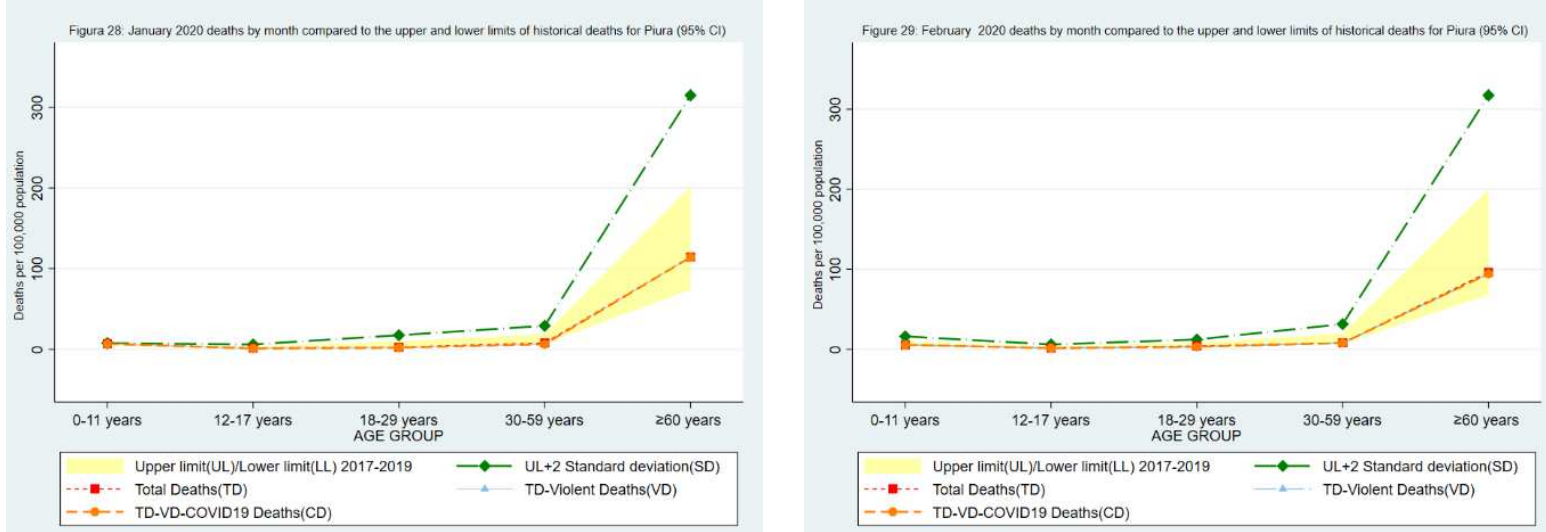

525
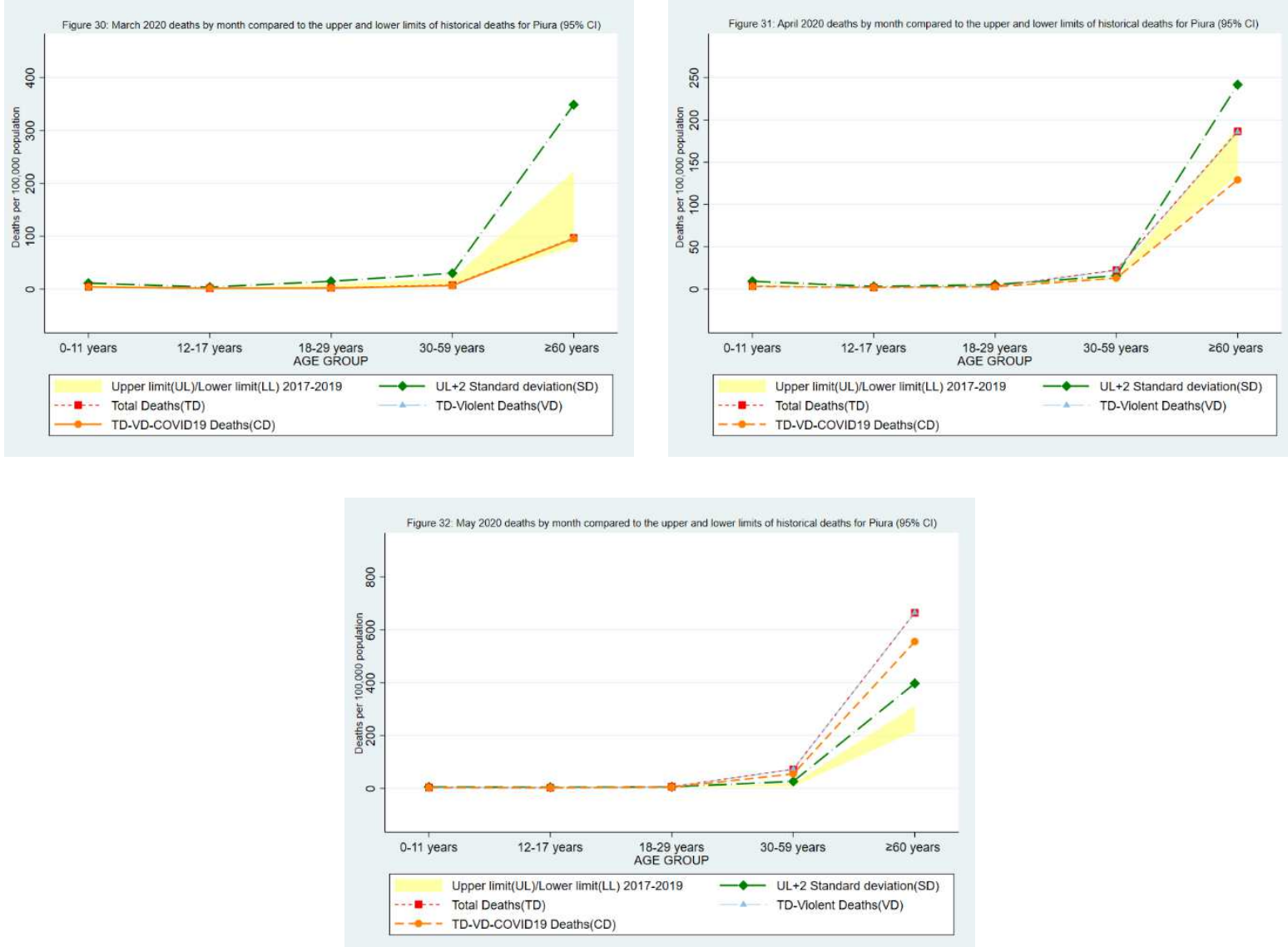


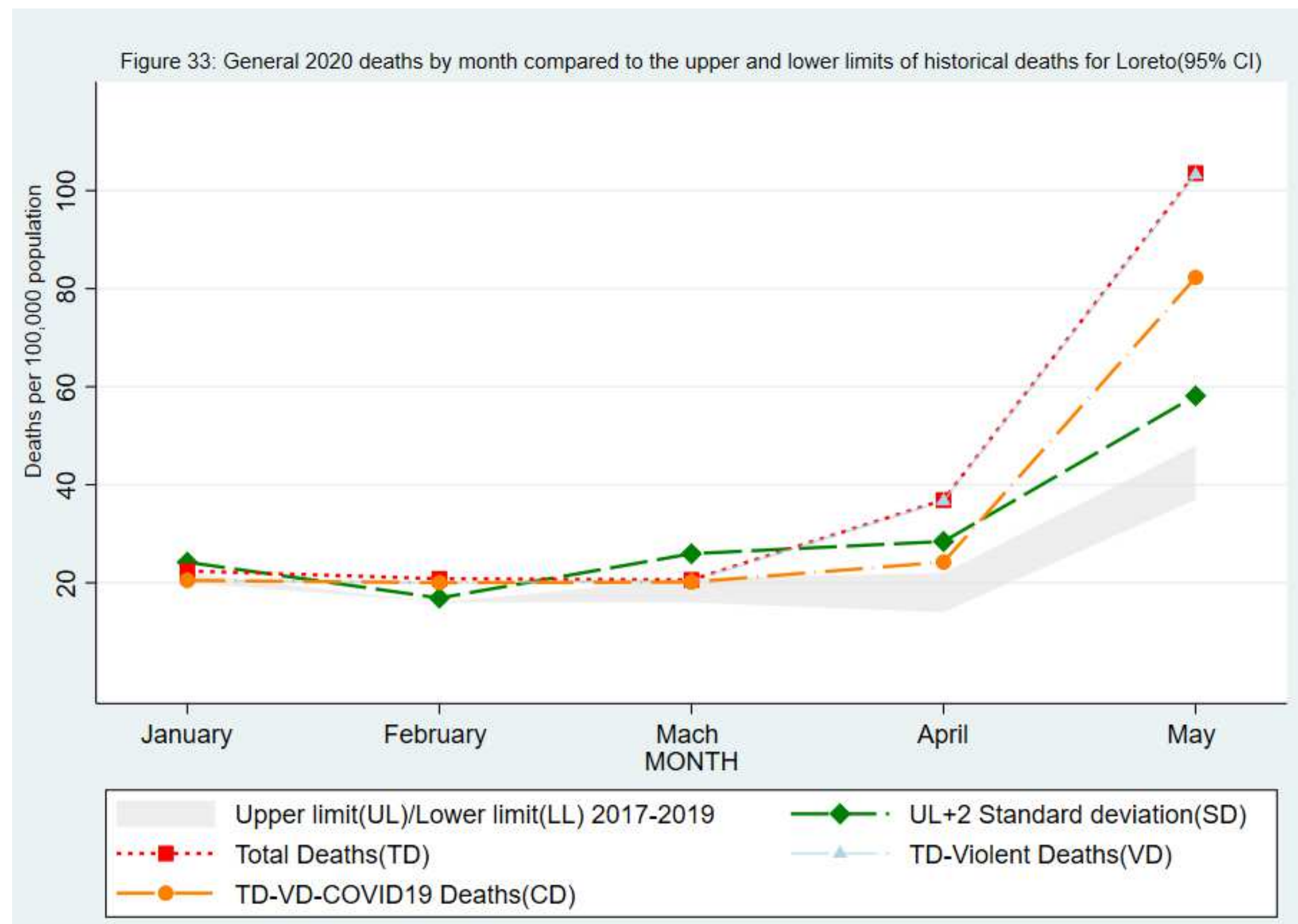



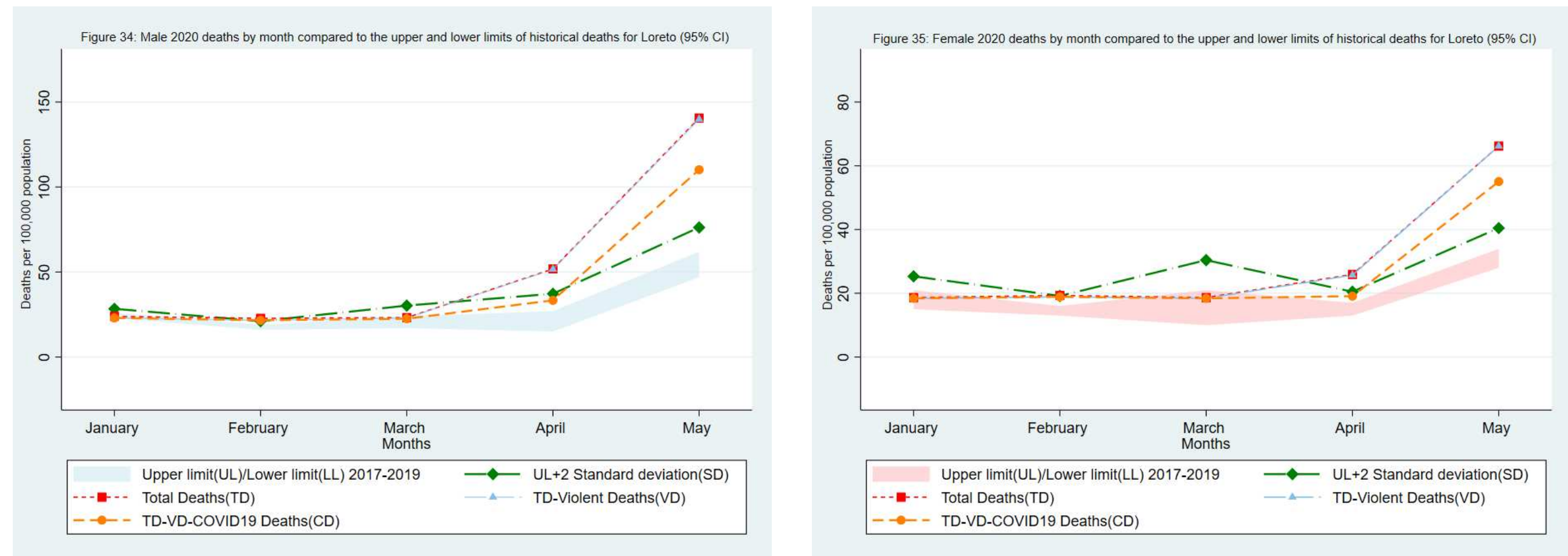

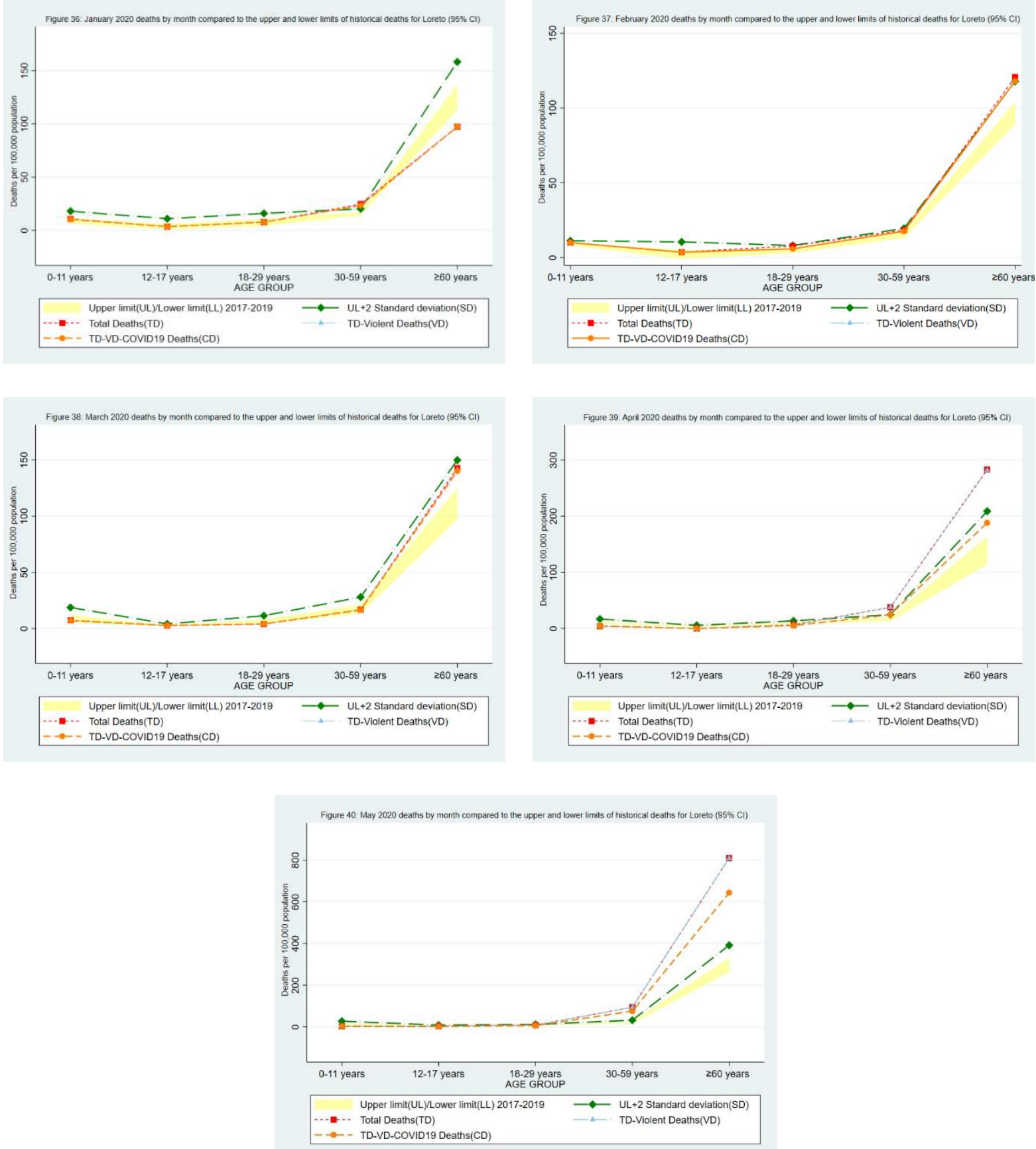


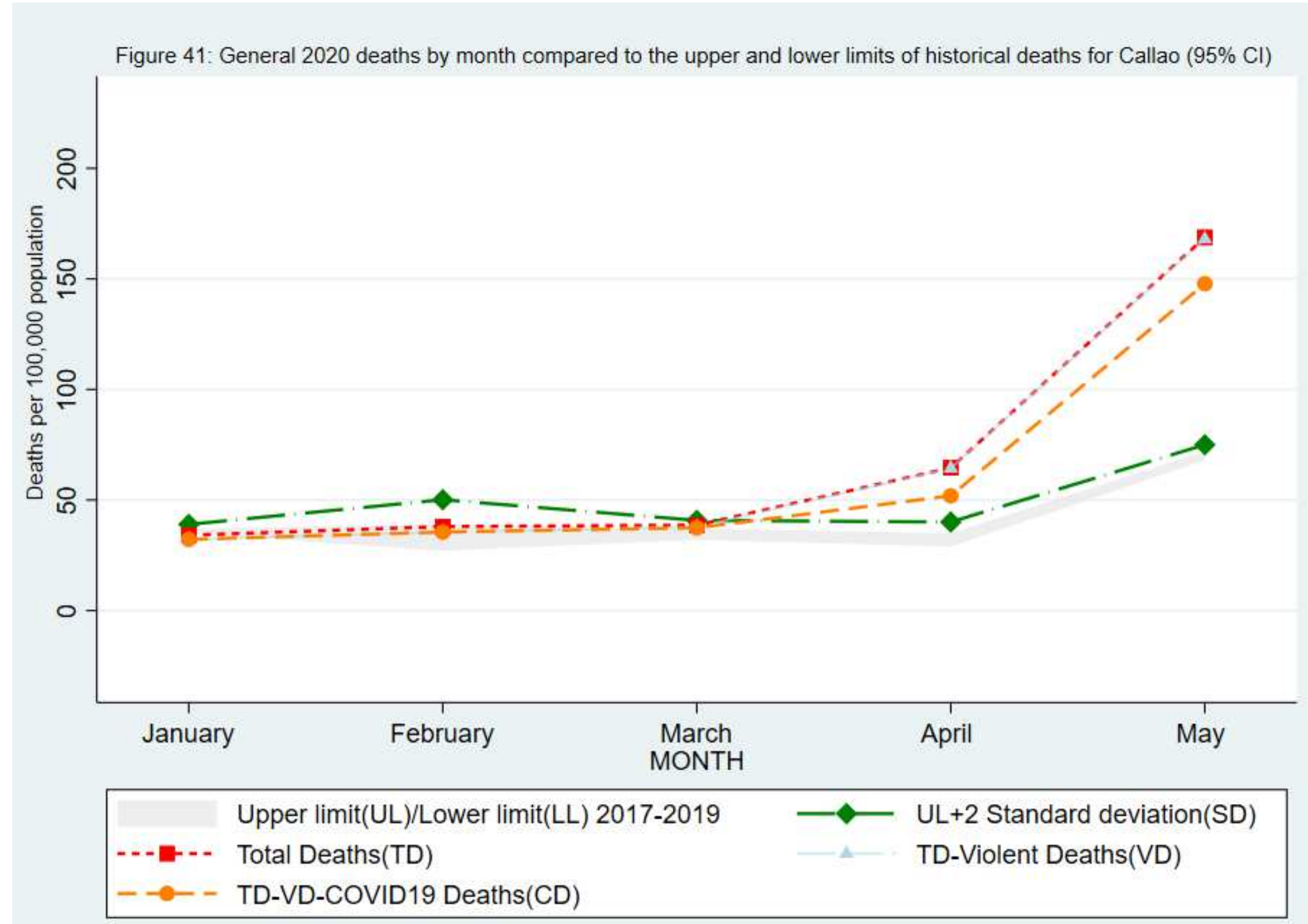



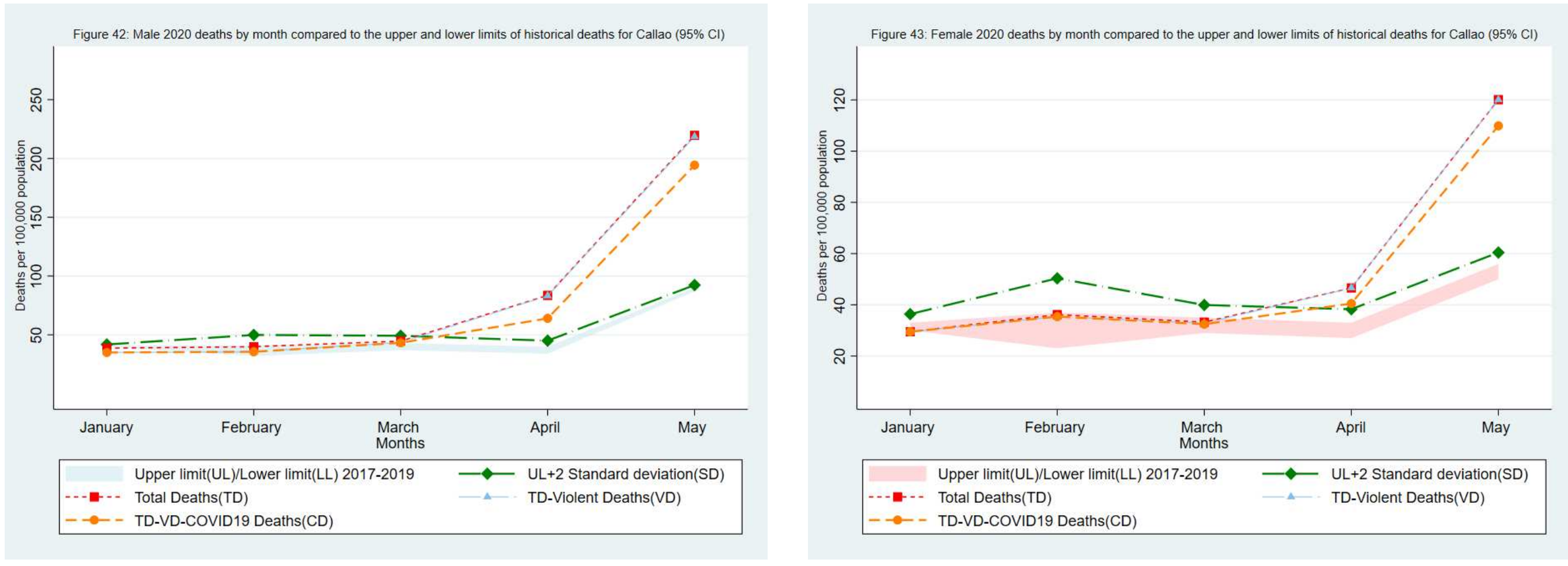

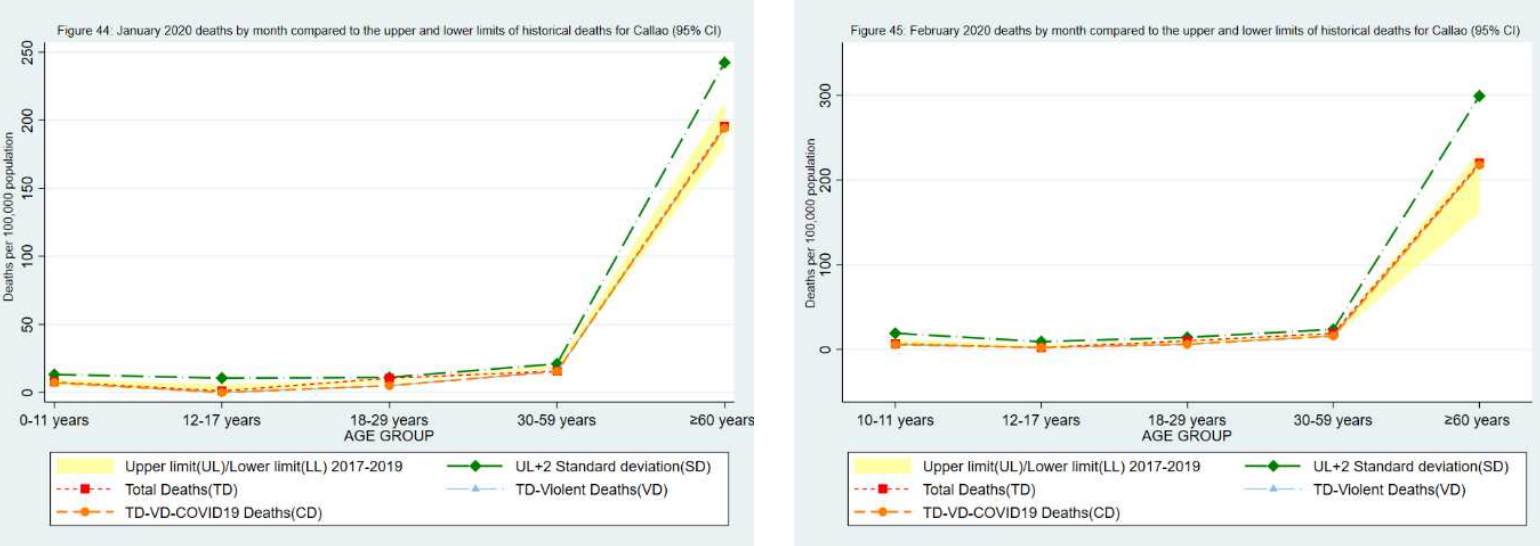

535
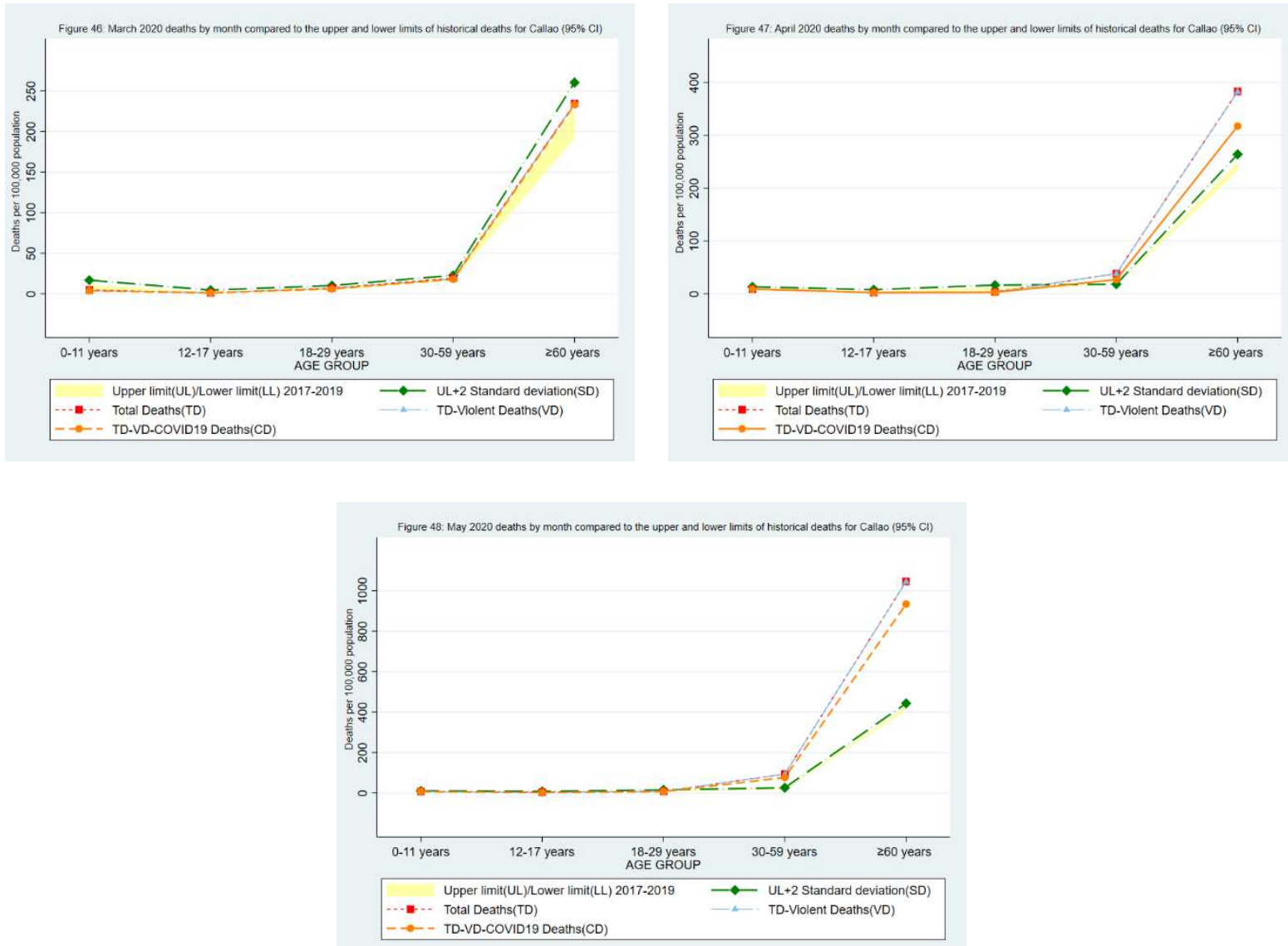


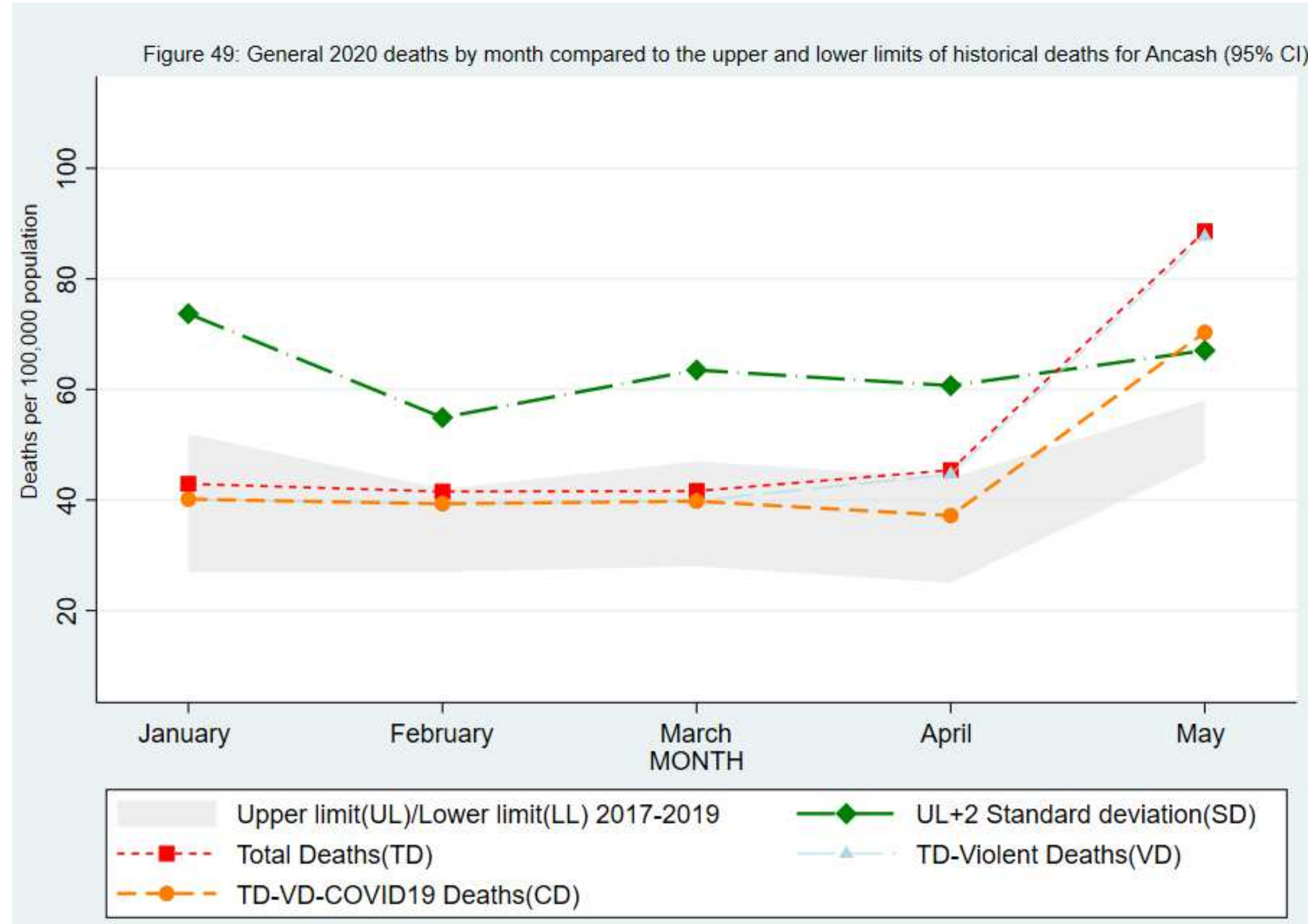



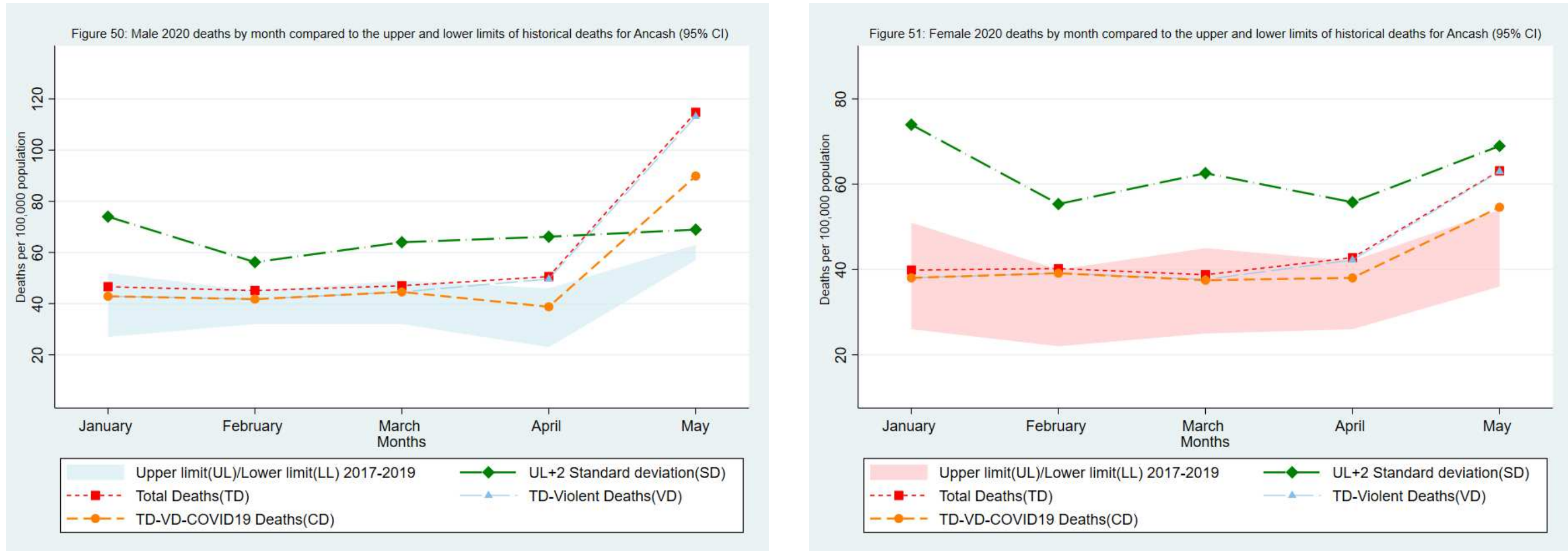

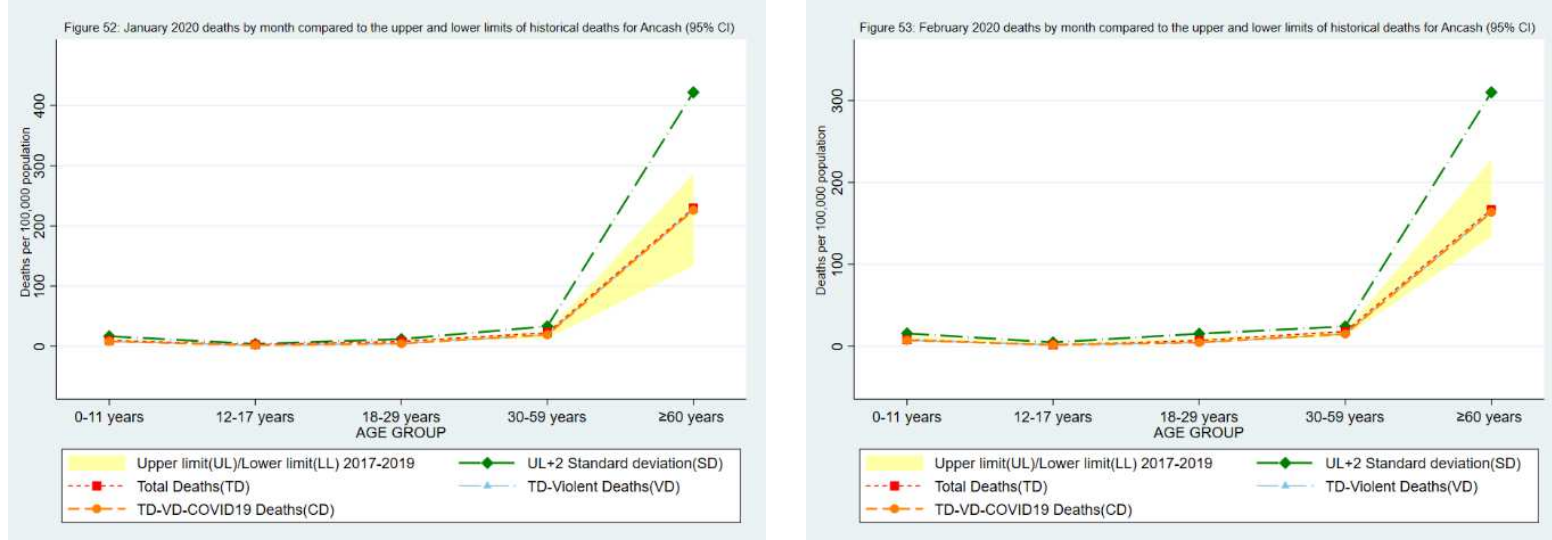

541
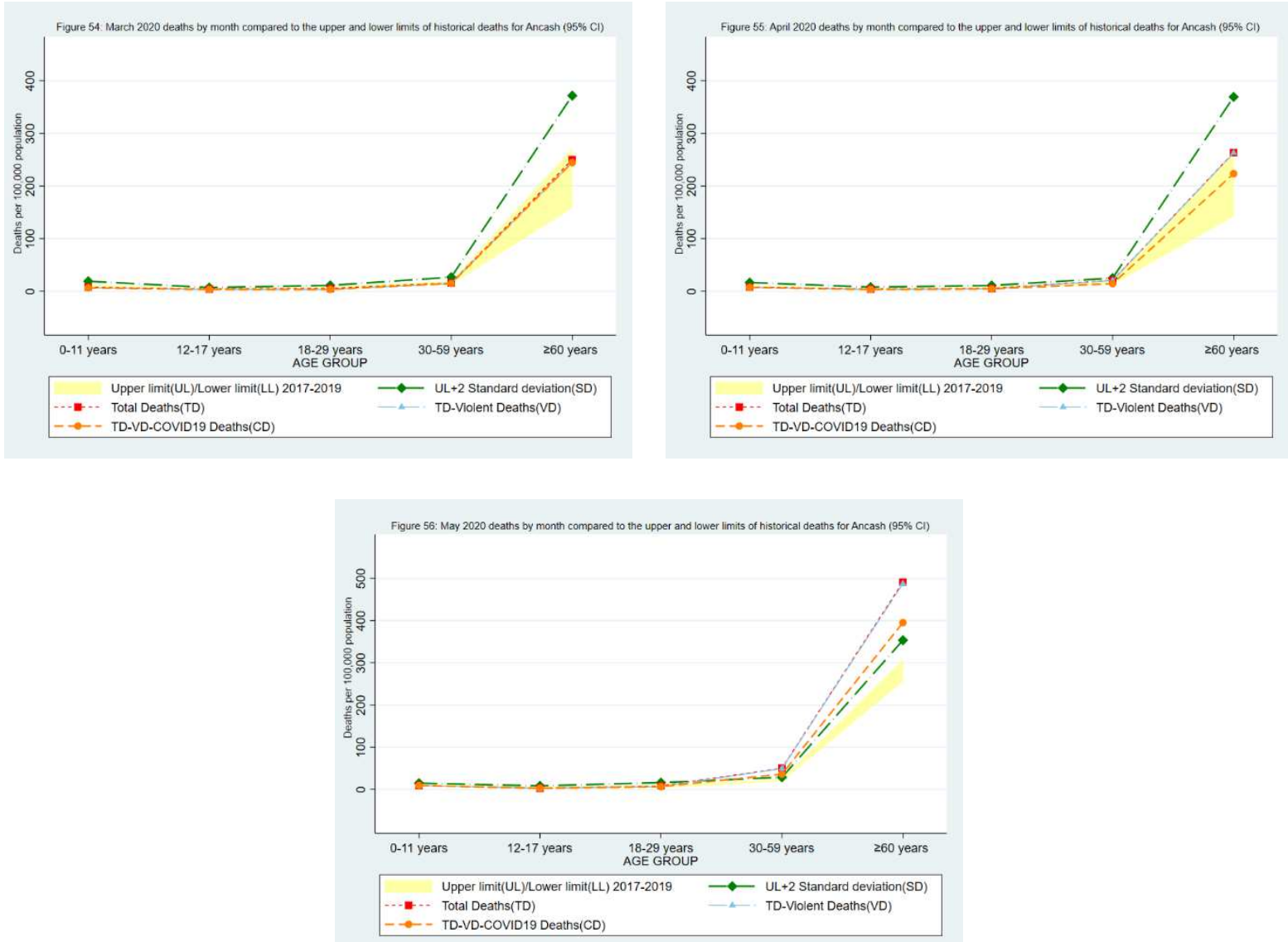


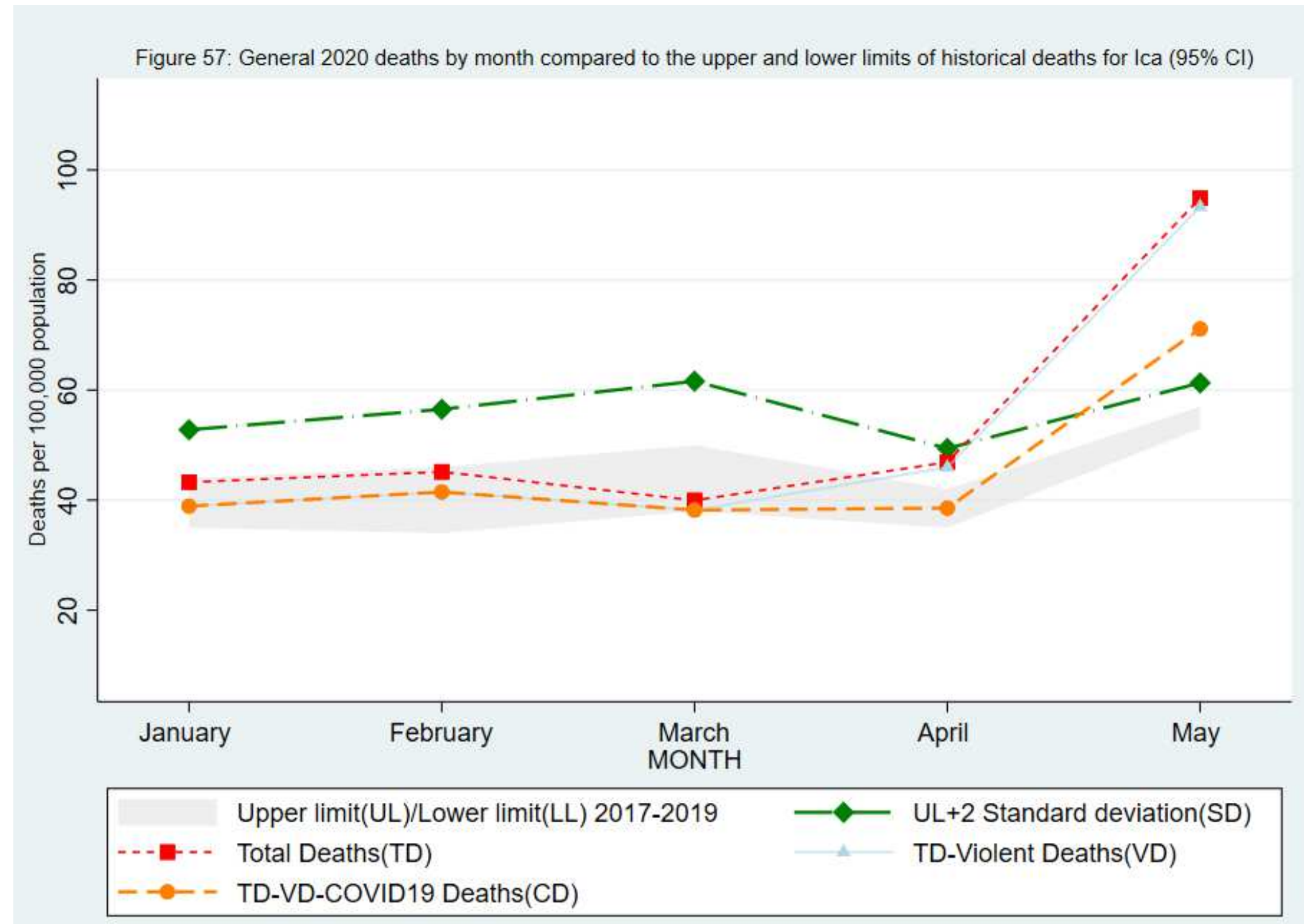



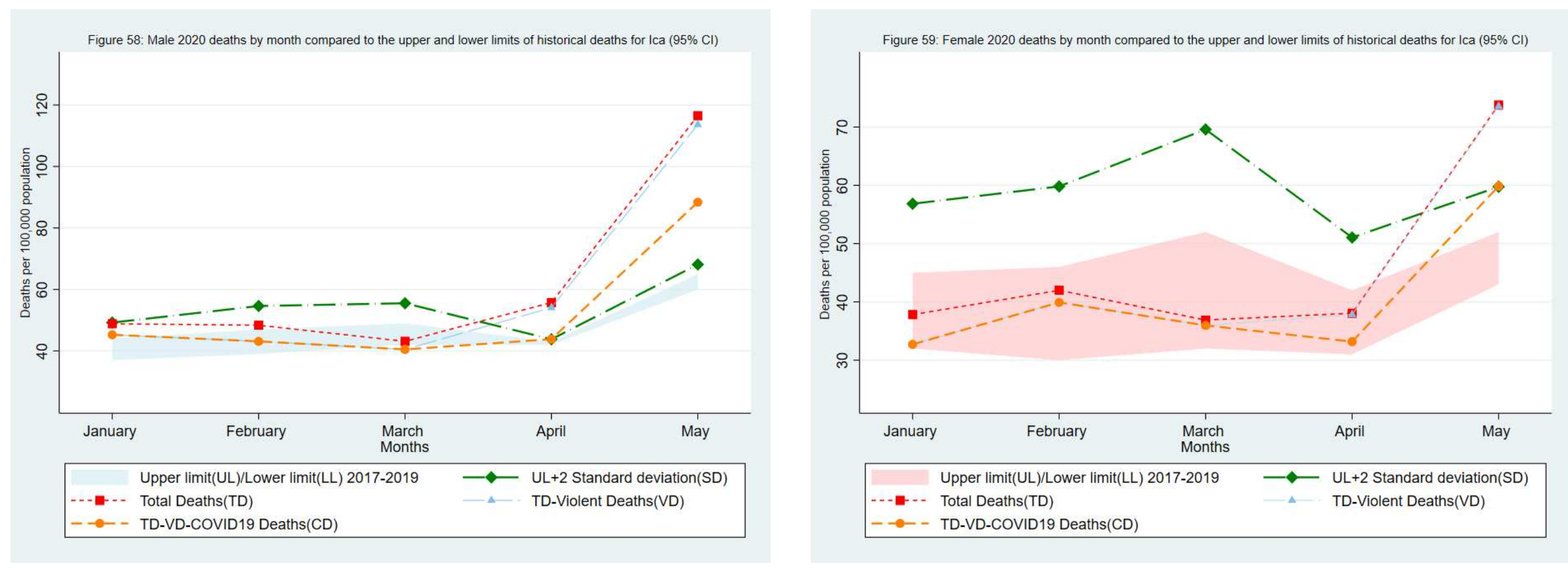

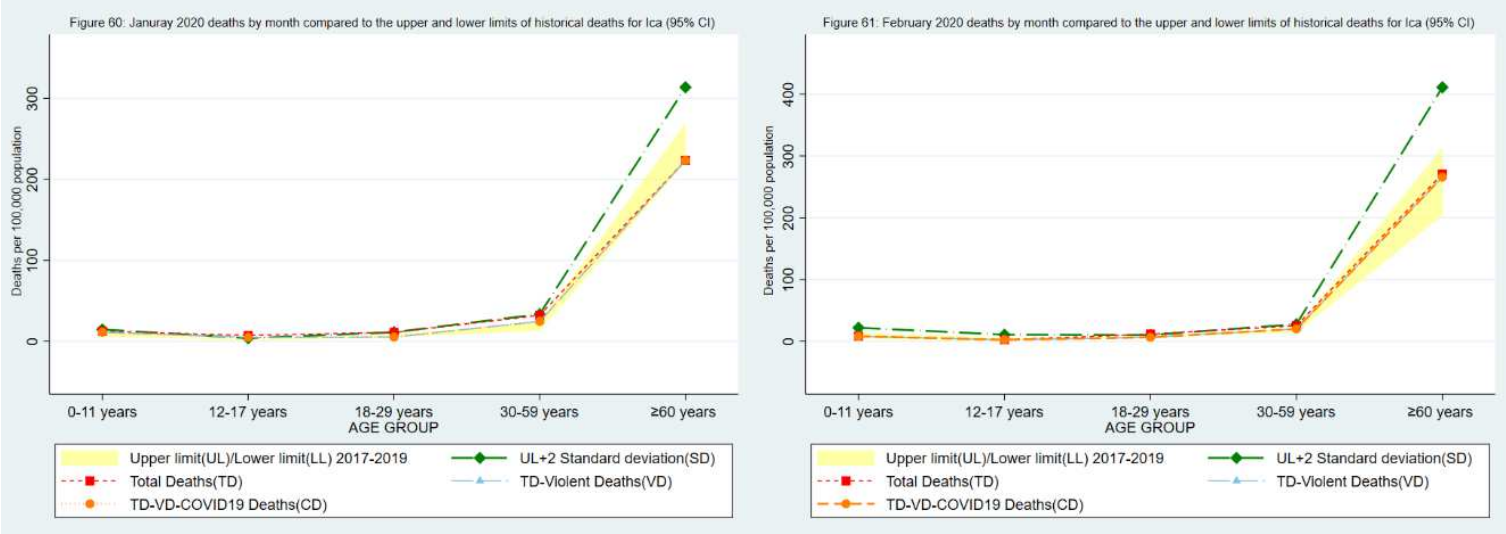

546
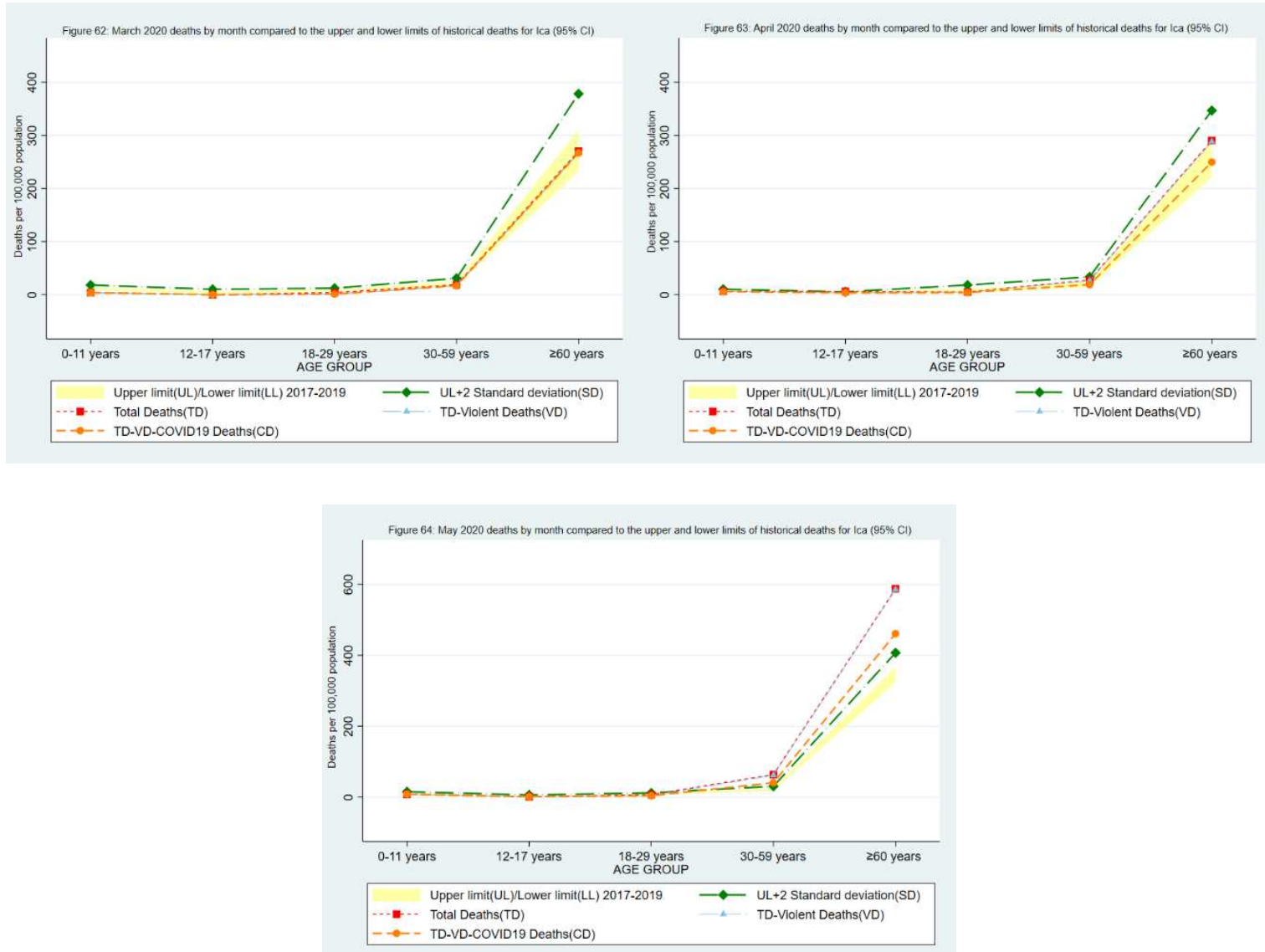


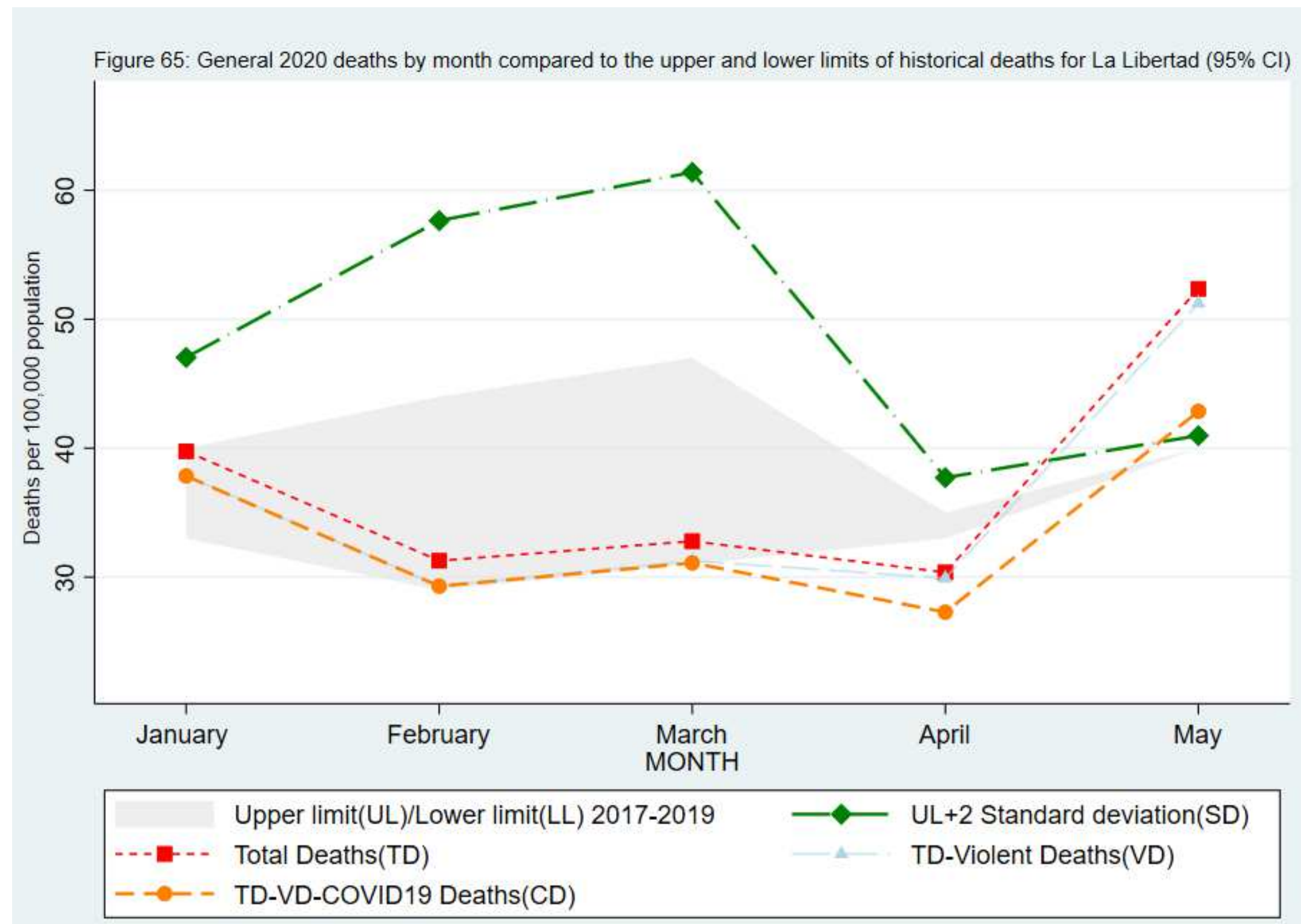



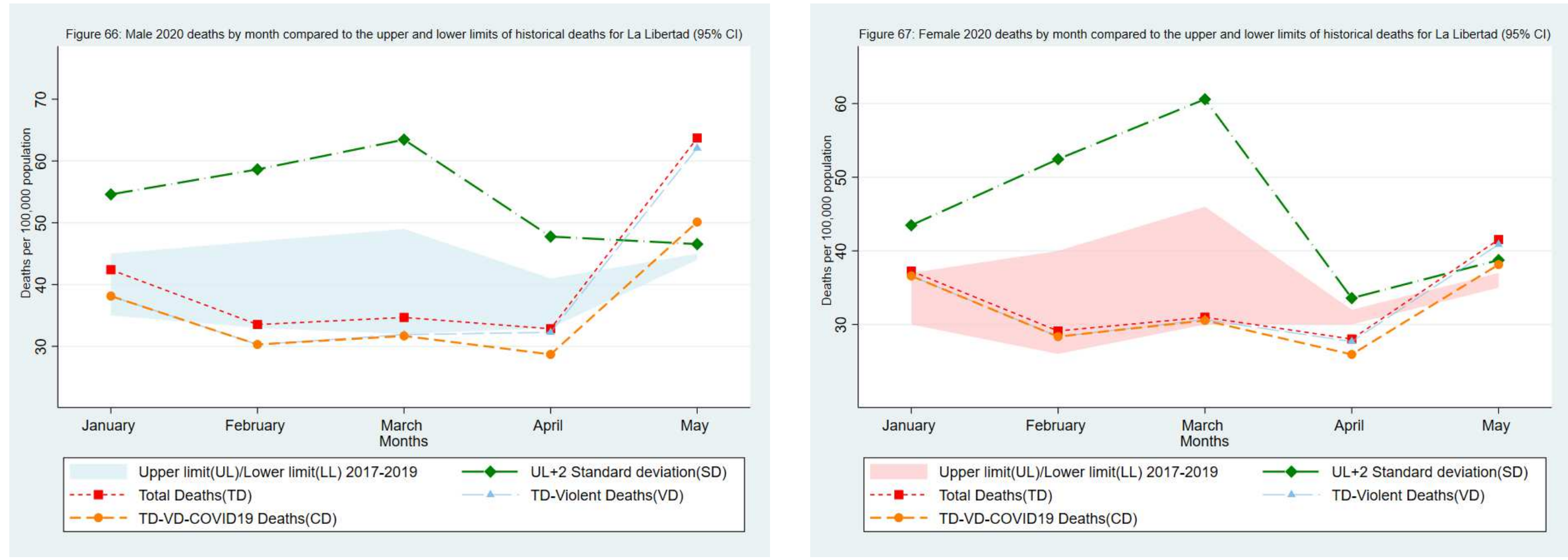

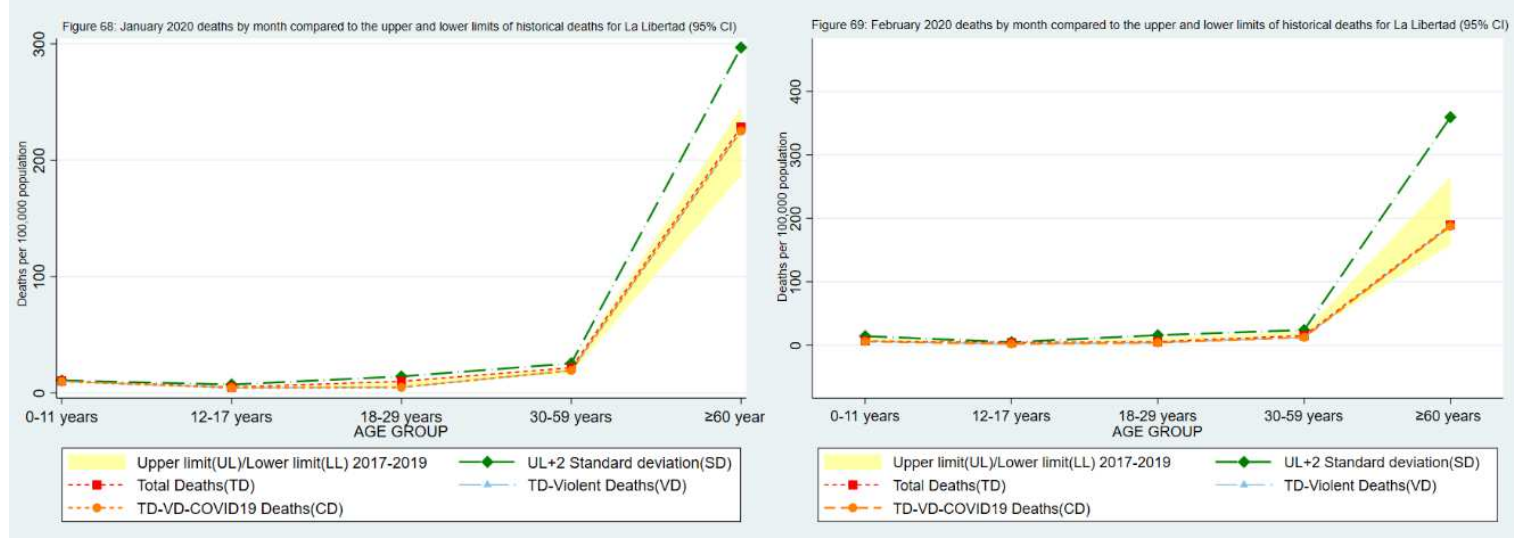

551
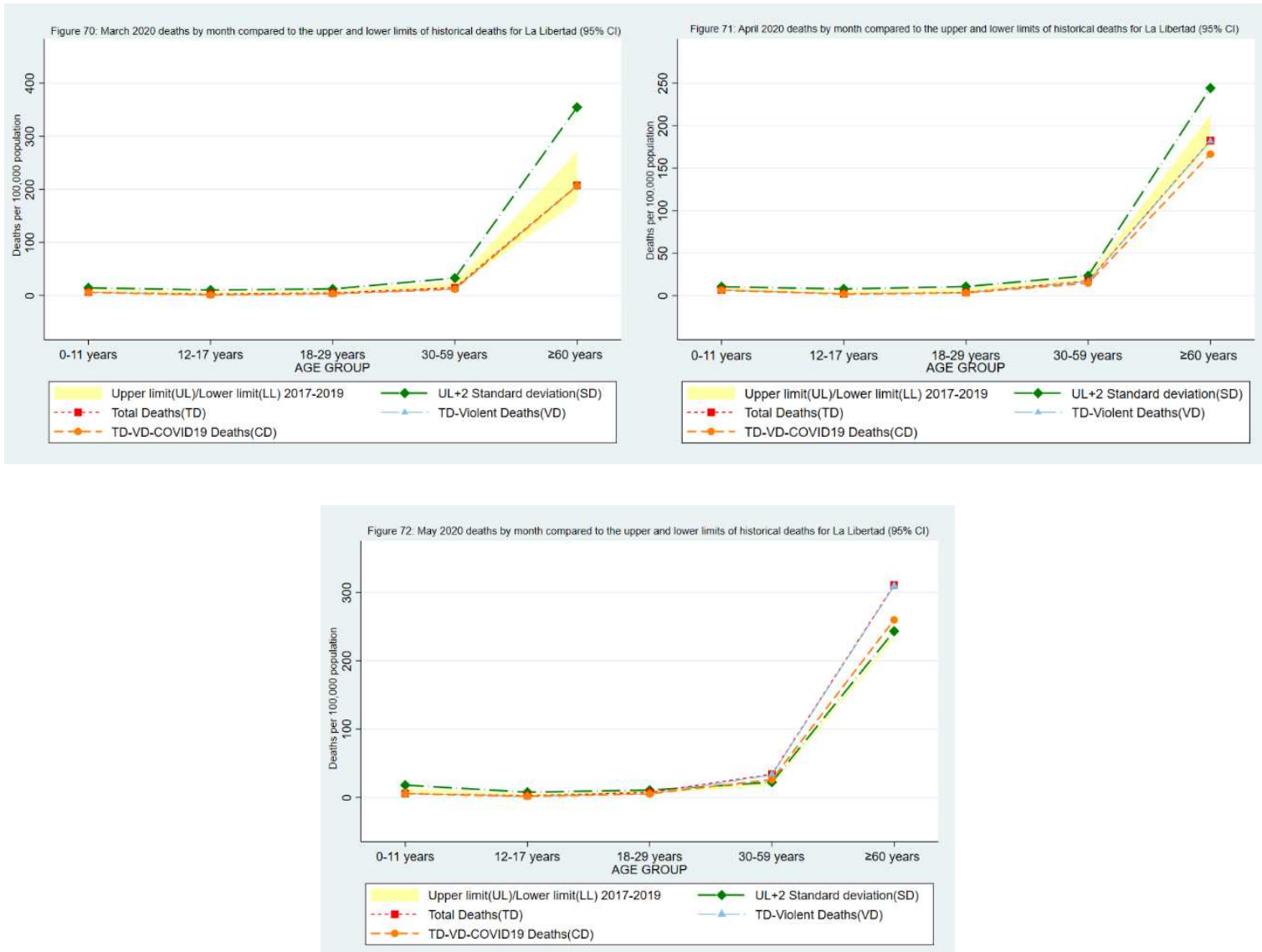


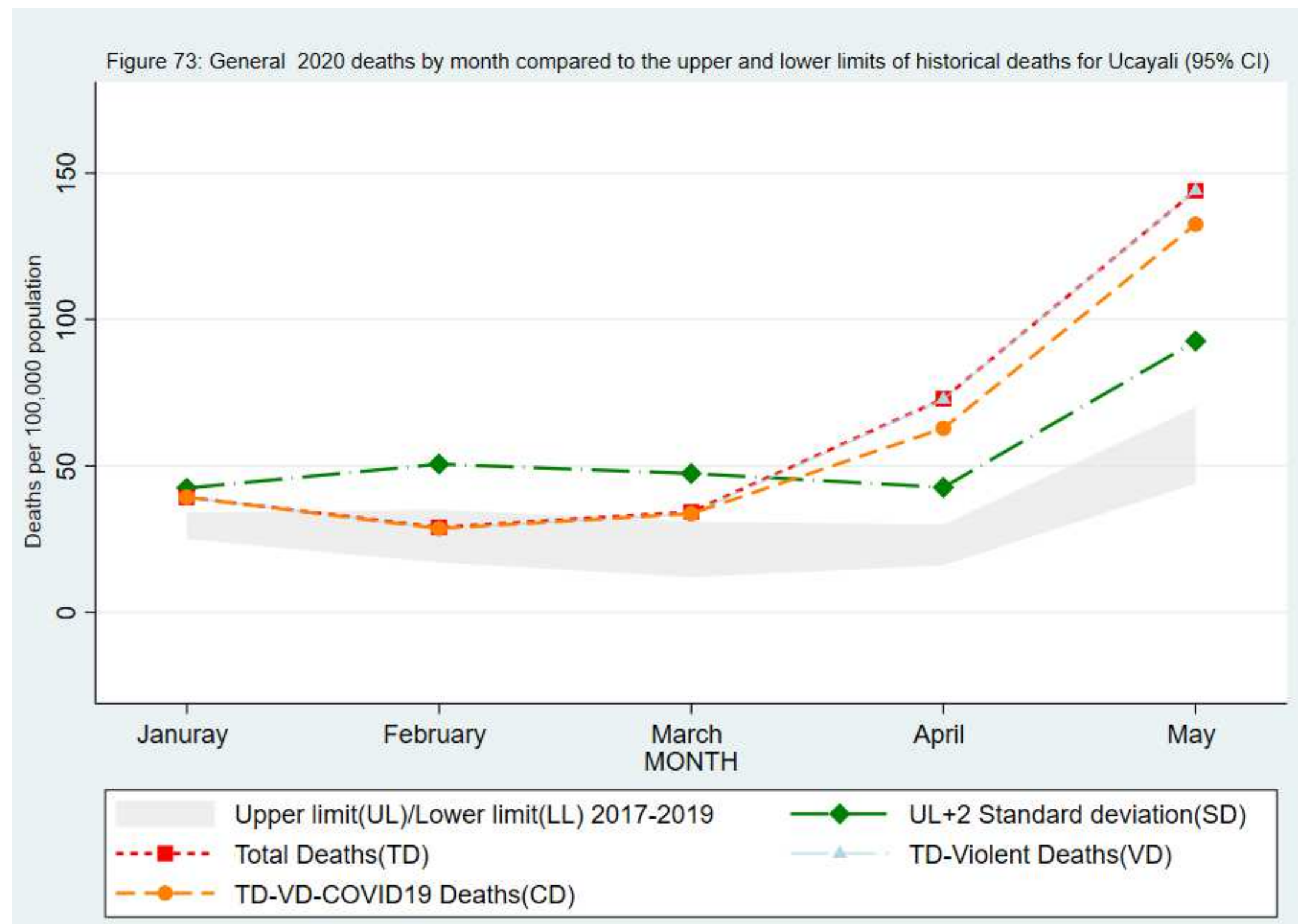



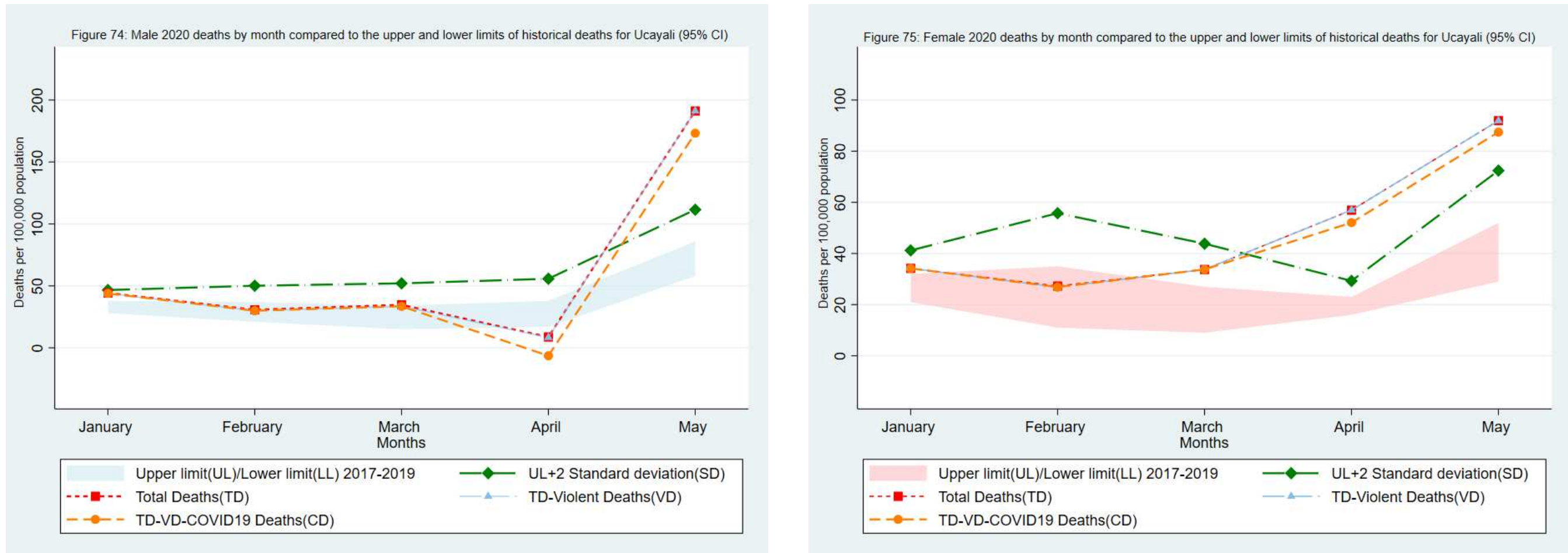

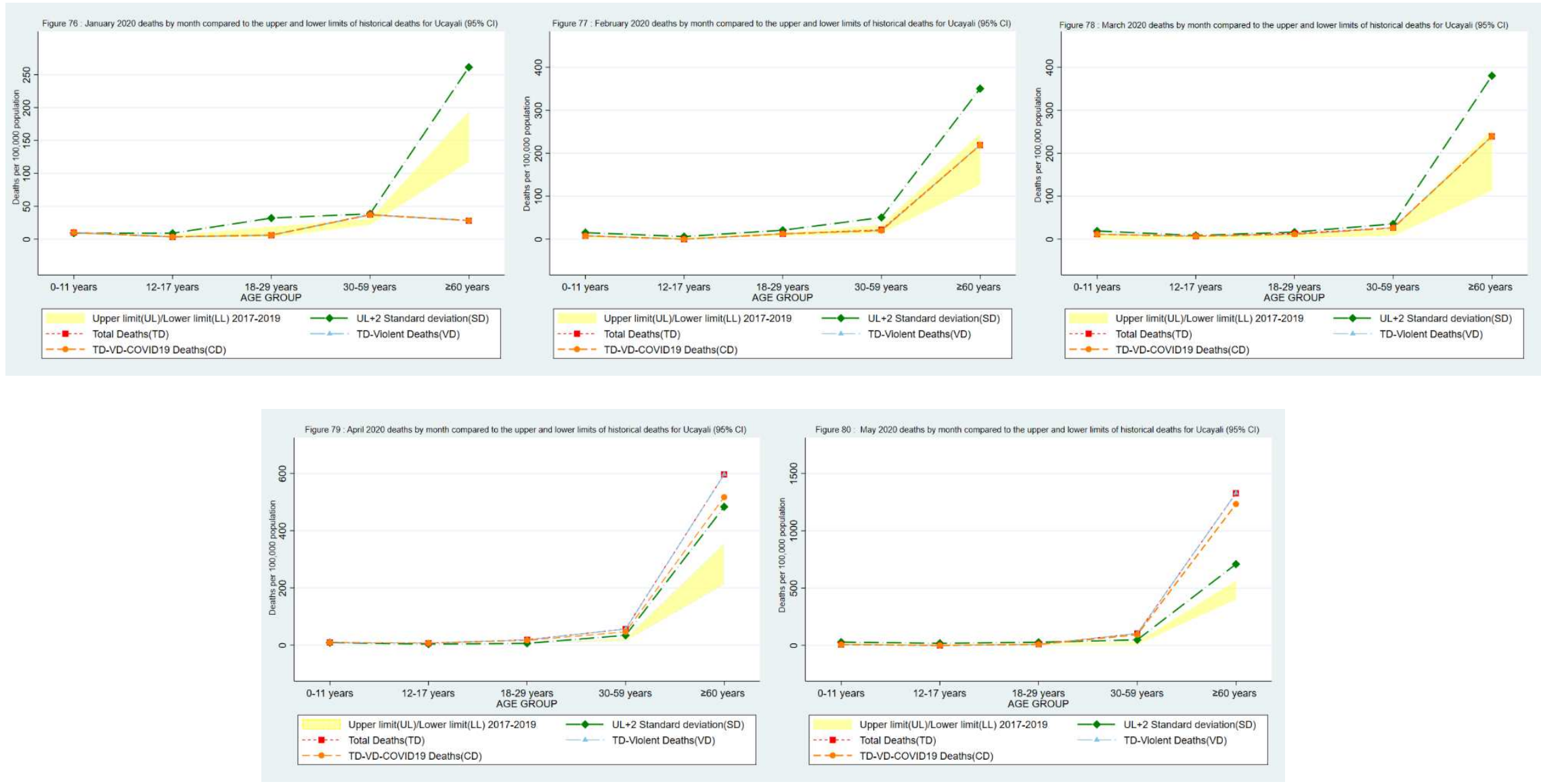


\section{REFERENCES}

559 1. World Health Organization. Timeline of WHO's response to COVID-19 [Internet]. Available from: https://www.who.int/news-room/detail/29-06-2020-covidtimeline

2. Ministerio de Salud del Perú. Casos positivos por COVID-19 [Internet]. 2020. Available from: https://www.datosabiertos.gob.pe/dataset/casos-positivos-por-covid-19ministerio-de-salud-minsa

564

3. Ministerio de Salud del Perú. Fallecidos por COVID-19 [Internet]. 2020. Available from: https://www.datosabiertos.gob.pe/dataset/fallecidos-por-covid-19-ministerio-de-saludminsa

4. Munayco C V, Tariq A, Rothenberg R, Soto-Cabezas GG, Reyes MF, Valle A, et al. Early transmission dynamics of COVID-19 in a southern hemisphere setting: Lima-Peru: February 29th-March 30th, 2020. Infect Dis Model [Internet]. 2020 May 12;5:338-45. Available from: https://pubmed.ncbi.nlm.nih.gov/32399507

5. Lisboa Bastos M, Tavaziva G, Abidi SK, Campbell JR, Haraoui L-P, Johnston JC, et al. Diagnostic accuracy of serological tests for covid-19: systematic review and metaanalysis. BMJ [Internet]. 2020 Jul 1;370:m2516. Available from: http://www.bmj.com/content/370/bmj.m2516.abstract

6. Simonsen L, Spreeuwenberg P, Lustig R, Taylor RJ, Fleming DM, Kroneman M, et al. Global Mortality Estimates for the 2009 Influenza Pandemic from the GLaMOR Project: A Modeling Study. PLOS Med [Internet]. 2013 Nov 26;10(11):e1001558. Available from: https://doi.org/10.1371/journal.pmed.1001558

7. Zheng Z, Peng F, Xu B, Zhao J, Liu H, Peng J, et al. Risk factors of critical \& mortal COVID-19 cases: A systematic literature review and meta-analysis. J Infect [Internet]. 2020 Available from: http://www.sciencedirect.com/science/article/pii/S0163445320302346 
583 8. Instituto Nacional de Estadística e Informática. Censos Nacionales 2017: XII de Población, VII de Vivienda y III de Comunidades Indígenas [Internet]. 2017. Available from: https://censos2017.inei.gob.pe/redatam/

9. Instituto Nacional de Estadística e Informática. Perú: Enfermedades No Transmisibles y Transmisibles, 2018 [Internet]. 2019. Available from: https://www.inei.gob.pe/media/MenuRecursivo/publicaciones_digitales/Est/Lib1657/li bro.pdf

10. Escobar G, Matta J, Ayala R, Amado J. Características clinicoepidemiológicas de pacientes fallecidos por covid-19 en un hospital nacional de Lima, Perú . Vol. 20, Revista de la Facultad de Medicina Humana . scielo ; 2020. p. 180-5.

11. Fernando Mejía, Carlos Medina, Enrique Cornejo, Enrique Morello, Sergio Vásquez, Jorge Alave, Alvaro Schwalb GM. Características clínicas y factores asociados a mortalidad en pacientes adultos hospitalizados por COVID-19 en un hospital público de Lima, Perú. Prepr SciELO [Internet]. 2020; Available from: https://preprints.scielo.org/index.php/scielo/preprint/view/858

12. Oyesanya M, Lopez-Morinigo J, Dutta R. Systematic review of suicide in economic recession. World J psychiatry. 2015 Jun;5(2):243-54.

13. Reger MA, Stanley IH, Joiner TE. Suicide Mortality and Coronavirus Disease 2019-A Perfect Storm? JAMA Psychiatry [Internet]. 2020 Apr 10; Available from: https://doi.org/10.1001/jamapsychiatry.2020.1060

14. Magnani C, Azzolina D, Gallo E, Ferrante D, Gregori D. How Large Was the Mortality 605 Increase Directly and Indirectly Caused by the COVID-19 Epidemic? An Analysis on All-Causes Mortality Data in Italy. Int J Environ Res Public Health [Internet]. 2020 May 15;17(10):3452. Available from: https://pubmed.ncbi.nlm.nih.gov/32429172

15. Ministerio de Salud del Perú. Sistema Informático Nacional de Defunciones [Internet]. 

http://www.minsa.gob.pe/reunis/data/defunciones_registradas.asp

16. Leon DA, Shkolnikov VM, Smeeth L, Magnus P, Pechholdová M, Jarvis CI. COVID19: a need for real-time monitoring of weekly excess deaths. Lancet [Internet]. 2020/04/22. 2020 May 2;395(10234):e81-e81. Available from: https://pubmed.ncbi.nlm.nih.gov/32333839

17. Green HK, Andrews NJ, Bickler G, Pebody RG. Rapid estimation of excess mortality: nowcasting during the heatwave alert in England and Wales in June 2011. J Epidemiol Community Health. 2012 Oct;66(10):866-8.

18. Vieira A, Peixoto VR, Aguiar P, Abrantes A. Rapid estimation of excess mortality in times of COVID-19 in Portugal - Beyond reported deaths. medRxiv [Internet]. 2020 Jan $1 ; 2020.05 .14 .20100909$. Available

from: http://medrxiv.org/content/early/2020/05/19/2020.05.14.20100909.abstract

19. Instituto Nacional de Estadística e Informática. La población de Lima supera los nueve millones y medio de habitantes [Internet]. Lima; 2020. Available from: http://m.inei.gob.pe/media/MenuRecursivo/noticias/notadeprensa006.pdf

20. Defensoría del Pueblo. Estado de Emergencia Sanitaria: El problema de la informalidad laboral en una economía confinada [Internet]. Lima; 2020. Available from: https://www.defensoria.gob.pe/wp-content/uploads/2020/04/Serie-de-InformesEspeciales-Nº-02-2020-DP-Problema-de-la-informalidad-laboral-en-una-economiaconfinada.pdf

21. Bradbury-Jones C, Isham L. The pandemic paradox: The consequences of COVID-19 on domestic violence. J Clin Nurs [Internet]. 2020/04/22. 2020 Jul;29(13-14):2047-9. Available from: https://pubmed.ncbi.nlm.nih.gov/32281158

22. Calderon-Anyosa RJC, Kaufman JS. Impact of COVID-19 Lockdown Policy on 
Homicide, Suicide, and Motor Vehicle Deaths in Peru. medRxiv [Internet]. 2020 Jan $1 ; 2020.07 .11 .20150193$.

Available

from: http://medrxiv.org/content/early/2020/07/14/2020.07.11.20150193.abstract

23. Vasquez-Chavesta AZ, Morán-Mariños C, Rodrigo-Gallardo PK, Toro-Huamanchumo CJ. COVID-19 and dengue: Pushing the peruvian health care system over the edge. Travel Med Infect Dis [Internet]. 2020 Jun 26;36:101808. Available from: https://pubmed.ncbi.nlm.nih.gov/32599174

24. Centro Nacional de Epidemiologia P y C de E. Sala situacional para el Análisis de Situación de Salud - SE 27- 2020 [Internet]. Lima; 2020. Available from: https://www.dge.gob.pe/portal/docs/vigilancia/sala/2020/SE03/dengue.pdf

25. Michelozzi P, de'Donato F, Scortichini M, de Sario M, Noccioli F, Rossi P, et al. Mortality impacts of the coronavirus disease (COVID-19) outbreak by sex and age: Rapid mortality surveillance system, Italy, 1 February to 18 April 2020. Eurosurveillance. 2020;25(19):1-5.

26. Kontopantelis E, Mamas MA, Deanfield J, Asaria M, Doran T. Excess mortality in England and Wales during the first wave of the COVID-19 pandemic. medRxiv. 2020;2020.05.26.20113357.

27. Piccininni M, Rohmann JL, Foresti L, Lurani C, Kurth T. Use of all cause mortality to quantify the consequences of covid-19 in Nembro, Lombardy: Descriptive study. BMJ. $2020 ; 369$.

28. Weinberger DM, Chen J, Cohen T, Crawford FW, Mostashari F, Olson D, et al. Estimation of Excess Deaths Associated With the COVID-19 Pandemic in the United States, March to May 2020. JAMA Intern Med [Internet]. 2020;06520(May):E1-9. Available from: https://sci-hub.tw/downloads-ii/2020-0702/3c/10.1001@jamainternmed.2020.3391.pdf 
29. Freitas ARR, Medeiros NM, Frutuoso L, Beckedorff OA, Martin LMA, Coelho MMM, et al. Use of excess mortality associated with the COVID-19 epidemic as an epidemiological surveillance strategy - preliminary results of the evaluation of six Brazilian capitals. medRxiv [Internet]. 2020;2020.05.08.20093617. Available from: http://medrxiv.org/lookup/doi/10.1101/2020.05.08.20093617

30. Liu Y, Mao B, Liang S, Yang J-W, Lu H-W, Chai Y-H, et al. Association between age and clinical characteristics and outcomes of COVID-19. Eur Respir J [Internet]. 2020 May 27;55(5):2001112. Available from: https://pubmed.ncbi.nlm.nih.gov/32312864

31. DeBiasi RL, Song X, Delaney M, Bell M, Smith K, Pershad J, et al. Severe COVID-19 in Children and Young Adults in the Washington, DC Metropolitan Region. J Pediatr [Internet]. 2020 May 13;223:199-203.e1. Available from: https://pubmed.ncbi.nlm.nih.gov/32405091

32. Centers for Disease Control and Prevention. Coronavirus Disease 2019: People of Any Age with Underlying Medical Conditions [Internet]. US; Available from: https://www.cdc.gov/coronavirus/2019-ncov/need-extra-precautions/people-withmedical-conditions.html

33. Bray F, Ferlay J, Soerjomataram I. Global Cancer Statistics 2018: GLOBOCAN Estimates of Incidence and Mortality Worldwide for 36 Cancers in 185 Countries. 2018;394-424.

34. Sánchez-Moreno F. The national health system in Peru. Rev Peru Med Exp Salud Publica. 2014;31(4):747-53.

35. The World Bank. Current health expenditure (\% of GDP) - Latin America \& Caribbean [Internet]. 2017. Available from: https://data.worldbank.org/indicator/SH.XPD.CHEX.GD.ZS?locations=ZJ

36. Lai AG, Pasea L, Banerjee A, Denaxas S, Katsoulis M, Chang WH, et al. Estimating 
excess mortality in people with cancer and multimorbidity in the COVID-19 emergency. medRxiv [Internet]. 2020;2020.05.27.20083287. Available from: https://www.medrxiv.org/content/10.1101/2020.05.27.20083287v1

37. Figueroa J, Brennan P, Theodoratou E, Poon M, Purshouse K, Din F, et al. Trends in excess cancer and cardiovascular deaths in Scotland during the COVID-19 pandemic 30 December 2019 to 20 April 2020. medRxiv [Internet]. 2020 Jan 1;2020.05.02.20086231. Available

from: http://medrxiv.org/content/early/2020/05/06/2020.05.02.20086231.abstract

38. Dale, B. ; Stylianou N. Coronavirus: What is the true death toll of the pandemic? British Broadcasting Corporation (BBC) [Internet]. Available from: https://www.bbc.com/news/world-53073046

39. Steven Bernard, David Blood, John Burn-Murdoch, Max Harlow, Caroline Nevitt, Alan Smith CT and AW. Coronavirus tracked: the latest figures as countries start to reopen. Finantial times [Internet]. 2020; Available from: https://raw.githubusercontent.com/Financial-Times/coronavirus-excess-mortalitydata/master/data/ft_excess_deaths.csv

40. Felix-Cardoso J, Vasconcelos H, Rodrigues P, Cruz-Correia R. Excess mortality during COVID-19 in five European countries and a critique of mortality analysis data. medRxiv [Internet]. 2020 Jan 1;2020.04.28.20083147. Available from: http://medrxiv.org/content/early/2020/05/02/2020.04.28.20083147.abstract

41. Docherty K, Butt J, de Boer R, Dewan P, Koeber L, Maggioni A, et al. Excess deaths during the Covid-19 pandemic: An international comparison. medRxiv [Internet]. 2020 Jan $1 ; 2020.04 .21 .20073114$. Available from: http://medrxiv.org/content/early/2020/05/13/2020.04.21.20073114.abstract

42. Wetzler HP, Wetzler EA. COVID-19 Excess Deaths in the United States, New York 
City, and Michigan During April 2020. medRxiv [Internet]. 2020;2020.04.02.20051532.

709 Available

from:

http://medrxiv.org/content/early/2020/05/19/2020.04.02.20051532.abstract 